\title{
On spatial and material covariant balance laws in elasticity
}

\author{
Arash Yavaria) \\ School of Civil and Environmental Engineering, Georgia Institute of Technology, \\ Atlanta, Georgia 30332 \\ Jerrold E. Marsden and Michael Ortiz \\ Division of Engineering and Applied Science, California Institute of Technology, \\ Pasadena, California 91125
}

(Received 6 January 2006; accepted 23 February 2006; published online 28 April 2006)

This paper presents some developments related to the idea of covariance in elasticity. The geometric point of view in continuum mechanics is briefly reviewed. Building on this, regarding the reference configuration and the ambient space as Riemannian manifolds with their own metrics, a Lagrangian field theory of elastic bodies with evolving reference configurations is developed. It is shown that even in this general setting, the Euler-Lagrange equations resulting from horizontal (referential) variations are equivalent to those resulting from vertical (spatial) variations. The classical Green-Naghdi-Rivilin theorem is revisited and a material version of it is discussed. It is shown that energy balance, in general, cannot be invariant under isometries of the reference configuration, which in this case is identified with a subset of $\mathbb{R}^{3}$. Transformation properties of balance of energy under rigid translations and rotations of the reference configuration is obtained. The spatial covariant theory of elasticity is also revisited. The transformation of balance of energy under an arbitrary diffeomorphism of the reference configuration is obtained and it is shown that some nonstandard terms appear in the transformed balance of energy. Then conditions under which energy balance is materially covariant are obtained. It is seen that material covariance of energy balance is equivalent to conservation of mass, isotropy, material Doyle-Ericksen formula and an extra condition that we call configurational inviscidity. In the last part of the paper, the connection between Noether's theorem and covariance is investigated. It is shown that the DoyleEricksen formula can be obtained as a consequence of spatial covariance of Lagrangian density. Similarly, it is shown that the material Doyle-Ericksen formula can be obtained from material covariance of Lagrangian density. (C) 2006 American Institute of Physics. [DOI: 10.1063/1.2190827]

\section{INTRODUCTION}

Invariance plays an important role in mechanics and in physics. In any continuum theory one has some conservation laws; i.e., quantities that are constant in time, such as mass and energy or balance laws, such as balance of linear and angular momentum. One way of building a continuum theory is to postulate these conservation or balance laws. On the other hand, as we shall recall later, conservation laws and even balance laws can be obtained as a result of postulating invariance of a quantity such as energy or Lagrangian density, under some group of transformations.

Traditionally, continuum mechanics is developed using Euclidean space as the ambient space. This has been motivated by the engineering applications of continuum mechanics and the general tendency of the engineering community to work with the simplest possible spaces. This is of

\footnotetext{
a) Author to whom correspondence should be addressed. Electronic mail: arash.yavari@ce.gatech.edu 
course useful and the implicit simplifying assumptions of continuum mechanics have made it applicable to many problems of practical importance. However, being restricted to the misleading and rigid structure of Euclidean space, one should expect to lose important geometric information. For example, for many years there were debates on different stress rates and whether one stress rate is "more objective" than the other one. Putting continuum mechanics in the right geometric setting, one can clearly see that different stress rates in the literature are simply different representations of the same Lie derivative. ${ }^{28}$

Another basic example of the lack of geometry in the traditional formulation of continuum mechanics is the dependence of the well-known balance of linear and angular momenta on the linear structure of Euclidean space. These laws are written in terms of integrals of some vector fields. Of course, integrating a vector field has no intrinsic meaning and is dependent on a linear structure or a specific coordinate choice. One can argue that a geometric point of view has proven useful in, for example, building systematic numerical schemes as well as in bridging length and time scales. For example, geometry has proven useful in Refs. 25, 6, and 3, although much remains to be done in the future.

Following Einstein's idea that physical laws should not depend on any particular choice of coordinate representation of ambient spaces, Marsden and Hughes ${ }^{28}$ developed a covariant theory of elasticity building on ideas originated from the work of Naghdi, Green and Rivilin. ${ }^{19}$ This work starts from balance of energy, which makes sense intrinsically as it is written in terms of integrals of scalar fields (or more precisely 3-forms). Then they postulate that balance of energy is invariant under arbitrary diffeomorphisms of the ambient space. They observe that this invariance assumption gives all the usual balance laws plus the Doyle-Ericksen formula that relates the stress and the metric tensor.

Our motivation for studying spatial and material covariant balance laws was to gain a better understanding of the geometry of configurational forces, which are forces that act in the reference configuration. One may ask the following question. What are the consequences of postulating that balance of energy is materially covariant? In the process of answering this question we discovered that such invariance cannot hold in general and this led us to obtain formulas for the way in which balance of energy transforms under material diffeomorphisms. In this paper we also study the connection between spatial and material covariance with Noether's theorem. It will be shown that spatial and material covariance of a Lagrangian density lead to the spatial and material forms of the Doyle-Ericksen formula, respectively.

As was mentioned, one of our motivations for this study was to initiate a geometric study of configurational forces. These forces and their balance laws are important in formulating the evolution of defects in solids in the setting of continuum mechanics. Driving (configurational, material and so forth) forces in continuum mechanics were introduced by Eshelby, ${ }^{13-15}$ and many researchers have studied them from different points of view. We mention the work of Knowles, ${ }^{22}$ Abeyaratneh and Knowles ${ }^{1,2}$ on driving force on a phase interface, Gurtin's work ${ }^{20,21}$ on configurational forces by postulating new balance laws, the work of Maugin ${ }^{31,32}$ and Maugin and Trimarco $^{33}$ on pull-back of balance of standard linear momentum to the reference configuration, etc. However, even after more than five decades after Eshelby's original work there does not seem to be a consensus on the nature of configurational forces and their exact role in continuum mechanics and there are still some controversies in the literature. We believe that the geometric ideas in this paper may be helpful in this direction.

This paper is organized as follows. The geometry of continuum mechanics is reviewed in Sec. II. The Lagrangian field theory of elastic bodies with evolving reference configurations is presented in Sec. III, where deformed bodies and their reference configurations are treated as Riemannian manifolds. Using this setting, the classical Green-Naghdi-Rivilin theorem and a new material version of it are discussed in Sec. IV. Spatial covariant energy balance is revisited in Sec. V. In Sec. VI we obtain the transformation (push-forward) of energy balance under an arbitrary material diffeomorphism. Then, we investigate the consequences of material covariance of energy balance. Section VII studies the connection between covariance and Noether's theorem. It is 
shown that spatial and material covariance of a Lagrangian density result in spatial and material versions of the Doyle-Ericksen formula, respectively. Conclusions and future directions are given in Sec. VIII.

\section{GEOMETRY OF CONTINUUM MECHANICS}

This section recalls some notation from the geometric approach to continuum mechanics that will be needed. It is assumed that the reader is familiar with the basic ideas; refer to, for example, Marsden and Hughes ${ }^{28}$ for details. See also Refs. 30 and 29.

If $M$ is a smooth $n$-manifold, the tangent space to $M$ at a point $p \in M$ is denoted $T_{p} M$, while the whole tangent bundle is denoted TM.

We denote by $\mathfrak{B}$ a reference manifold for our body and by $\mathcal{S}$ the space in which the body moves. We assume that $\mathfrak{B}$ and $\mathcal{S}$ are Riemannian manifolds with metrics denoted by $\mathbf{G}$ and $\mathbf{g}$, respectively. Local coordinates on $\mathfrak{B}$ are denoted by $X^{I}$ and those on $\mathcal{S}$ by $x^{i}$. The material body $\mathcal{B}$ is a subset of the material manifold, i.e., $\mathcal{B} \subset \mathfrak{B}$.

A deformation of the body is, for purposes of this paper, a $C^{1}$ embedding $\varphi: \mathcal{B} \rightarrow \mathcal{S}$. The tangent map of $\varphi$ is denoted $\mathbf{F}=T \varphi: T \mathcal{B} \rightarrow T \mathcal{S}$; in the literature it is often called the deformation gradient. In local charts on $\mathcal{B}$ and $\mathcal{S}$, the tangent map of $\varphi$ is given by the Jacobian matrix of partial derivatives of the components of $\varphi$, which we write as

$$
\mathbf{F}=T \varphi: T \mathcal{B} \rightarrow T \mathcal{S}, \quad T \varphi(X, \mathbf{V})=(\varphi(X), \mathbf{D} \varphi(X) \cdot \mathbf{V}) .
$$

If $F: \mathcal{B} \rightarrow \mathrm{R}$ is a $C^{1}$ scalar function, $X \in \mathcal{B}$ and $\mathbf{V}_{X} \in T_{X} \mathcal{B}$, then $\mathbf{V}_{X}[F]$ denotes the derivative of $F$ at $X$ in the direction of $\mathbf{V}_{X}$, i.e., $\mathbf{V}_{X}[F]=\mathbf{D} F(X) \cdot \mathbf{V}$. In local coordinates $\left\{X^{I}\right\}$ on $\mathcal{B}$,

$$
\mathbf{V}_{X}[F]=\frac{\partial F}{\partial X^{I}} V^{I} .
$$

For $f: \mathcal{S} \rightarrow \mathbb{R}$, the pull-back of $f$ by $\varphi$ is defined by

$$
\varphi^{*} f=f \circ \varphi \text {. }
$$

If $F: \mathcal{B} \rightarrow \mathbb{R}$, the push-forward of $F$ by $\varphi$ is defined by

$$
\varphi_{*} F=F \circ \varphi^{-1} .
$$

If $\mathbf{Y}$ is a vector field on $\mathcal{B}$, then $\varphi_{*} \mathbf{Y}=T \varphi \circ \mathbf{Y} \circ \varphi^{-1}$, or using the $\mathbf{F}$ notation, $\varphi_{*} \mathbf{Y}=\mathbf{F} \circ \mathbf{Y} \circ \varphi^{-1}$ is a vector field on $\varphi(\mathcal{B})$ called the push-forward of $\mathbf{Y}$ by $\varphi$. Similarly, if $\mathbf{y}$ is a vector field on $\varphi(\mathcal{B}) \subset \mathcal{S}$, then $\varphi^{*} \mathbf{y}=T\left(\varphi^{-1}\right) \circ \mathbf{y} \circ \varphi$ is a vector field on $\mathcal{B}$ and is called the pull-back of $\mathbf{y}$ by $\varphi$.

The cotangent bundle of a manifold $M$ is denoted $T^{*} M$ and the fiber at a point $p \in M$ (the vector space of one-forms at $p$ ) is denoted by $T_{p}^{*} M$. If $\beta$ is a one form on $\mathcal{S}$ (that is, a section of the cotangent bundle $T^{*} \mathcal{S}$ ), then the one-form on $\mathcal{B}$ defined as

$$
\left(\varphi^{*} \beta\right)_{X} \cdot \mathbf{V}_{X}=\beta_{\varphi(X)} \cdot\left(T \varphi \cdot \mathbf{V}_{X}\right)=\beta_{\varphi(X)} \cdot\left(\mathbf{F} \cdot \mathbf{V}_{X}\right)
$$

for $X \in \mathcal{B}$ and $\mathbf{V}_{X} \in T_{X} \mathcal{B}$, is called the pull-back of $\beta$ by $\varphi$. Likewise, the push-forward of a one-form $\alpha$ on $\mathcal{B}$ is the one form on $\varphi(\mathcal{B})$ defined by $\varphi_{*} \alpha=\left(\varphi^{-1}\right)^{*} \alpha$.

We can associate a vector field $\beta^{\sharp}$ to a one-form $\beta$ on a Riemannian manifold $M$ through the equation

$$
\left\langle\beta_{x}, \mathbf{v}_{x}\right\rangle=\left\langle\left\langle\beta_{x}^{\#}, \mathbf{v}_{x}\right\rangle\right\rangle_{x},
$$

where $\langle$,$\rangle denotes the natural pairing between the one-form \beta_{x} \in T_{x}^{*} M$ and the vector $\mathbf{v}_{x} \in T_{x} M$ and where $\left\langle\left\langle\beta_{x}^{\#}, \mathbf{v}_{x}\right\rangle\right\rangle_{x}$ denotes the inner product between $\beta_{x}^{\#} \in T_{x} M$ and $\mathbf{v}_{x} \in T_{x} M$. In coordinates, the components of $\beta^{\sharp}$ are given by $\beta^{i}=g^{i j} \beta_{i}$. 
Traditionally force is thought of as a vector field in the deformed configuration. For example, body force $\mathbf{B}$ per unit undeformed mass is a vector field on $\mathcal{S}$ and its associated one-form can be defined as

$$
\left\langle\beta_{x}, \delta \mathbf{w}\right\rangle=\langle\langle\mathbf{B}, \delta \mathbf{w}\rangle\rangle_{x}
$$

for all $\delta \mathbf{w} \in T_{x} \mathcal{S}$. The pull-back of $\beta$ is defined as

$$
\left\langle\left(\varphi^{*} \beta\right)_{X}, \delta \mathbf{W}\right\rangle_{X}=\left\langle\beta_{x}, \mathbf{F} \delta \mathbf{W}\right\rangle_{X}=\left\langle\left\langle\mathbf{B}, \mathbf{F} \delta \mathbf{W}_{X}\right\rangle\right\rangle_{X}=\left\langle\left\langle\mathbf{F}^{\top} \mathbf{B}, \delta \mathbf{W}_{X}\right\rangle\right\rangle_{X} .
$$

Therefore $\mathbf{F}^{\top} \mathbf{B}$ is the vector field associated with the pull-back of the one-form associated with $\mathbf{B}$. A type $\left(\begin{array}{l}p \\ q\end{array}\right)$-tensor at $X \in \mathcal{B}$ is a multilinear map,

$$
\mathrm{T}: \underbrace{T_{X}^{*} \mathcal{B} \times \cdots \times T_{X}^{*} \mathcal{B}}_{p \text { copies }} \times \underbrace{T_{X} \mathcal{B} \times \cdots \times T_{X} \mathcal{B}}_{q \text { copies }} \rightarrow \mathrm{R}
$$

$\mathbf{T}$ is said to be contravariant of order $p$ and covariant of order $q$. In a local coordinate chart,

$$
\mathbf{T}\left(\alpha^{1}, \ldots, \alpha^{p}, \mathbf{V}_{1}, \ldots, \mathbf{V}_{q}\right)=T^{i_{1} \cdots i_{p}}{ }_{j_{1} \cdots j_{q}} \alpha_{i_{1}}^{1} \cdots \alpha_{i_{p}}^{p} V_{1}^{j_{1}} \cdots V_{q}^{j_{q}},
$$

where $\alpha^{k} \in T_{X}^{*} \mathcal{B}$ and $\mathbf{V}^{k} \in T_{X} \mathcal{B}$.

Suppose $\varphi: \mathcal{B} \rightarrow \mathcal{S}$ is a regular map and $\mathbf{T}$ is a tensor of type $\left(\begin{array}{l}p \\ q\end{array}\right)$. Push-forward of $\mathbf{T}$ by $\varphi$ is denoted $\varphi_{*} \mathbf{T}$ and is a $\left(\begin{array}{l}p \\ q\end{array}\right)$-tensor on $\varphi(\mathcal{B})$ defined by

$$
\left(\varphi_{*} \mathbf{T}\right)(x)\left(\alpha^{1}, \ldots, \alpha^{p}, \mathbf{v}_{1}, \ldots, \mathbf{v}_{q}\right)=\mathbf{T}(X)\left(\varphi^{*} \alpha^{1}, \ldots, \varphi^{*} \alpha^{p}, \varphi^{*} \mathbf{v}_{1}, \ldots, \varphi^{*} \mathbf{v}_{q}\right),
$$

where $\alpha^{k} \in T_{x}^{*} \mathcal{S}, \mathbf{v}_{k} \in T_{x} \mathcal{S}, X=\varphi^{-1}(x), \varphi^{*}\left(\alpha^{k}\right) \cdot \mathbf{v}_{l}=\alpha^{k} \cdot\left(T \varphi \cdot \mathbf{v}_{l}\right)$ and $\varphi^{*}\left(\mathbf{v}_{l}\right)=T\left(\varphi^{-1}\right) \mathbf{v}_{l}$. Similarly, pullback of a tensor $\mathbf{t}$ defined on $\varphi(\mathcal{B})$ is given by $\varphi^{*} \mathbf{t}=\left(\varphi^{-1}\right) * \mathbf{t}$. In the setting of continuum mechanics push-forward and pull-back of tensors will have the following forms:

$$
\begin{aligned}
& \left(\varphi_{*} \mathbf{T}\right)^{i_{1} \cdots i_{p}}{ }_{p_{1} \cdots j_{q}}(x)=F^{i_{1}} I_{1}(X) \cdots F^{i_{p}} I_{p}(X) T^{I_{1} \cdots I_{p}}{ }_{J_{1} \cdots J_{q}}\left(F^{-1}\right)^{J_{1}} j_{j_{1}}(x) \cdots\left(F^{-1}\right)^{J_{j_{j}}}(x), \\
& \left(\varphi^{*} \mathbf{t}\right)^{I_{1} \cdots I_{p} \cdots J_{J} \cdots}(X)=\left(F^{-1}\right)^{I_{1}}{ }_{i_{1}}(x) \cdots\left(F^{-1}\right)^{I_{p}}{ }_{i_{p}}(x) t^{i_{1} \cdots i_{p} \cdots j_{j_{1}}} F^{j_{1}}{ }_{J_{1}}(X) \cdots F^{j_{q}} J_{q}(X) .
\end{aligned}
$$

A two-point tensor $\mathbf{T}$ of type $\left(\begin{array}{ll}q & q^{\prime} \\ p & p^{\prime}\end{array}\right)$ at $X \in \mathcal{B}$ over a map $\varphi: \mathcal{B} \rightarrow \mathcal{S}$ is a multilinear map,

$$
T: \underbrace{T_{X}^{*} \mathcal{B} \times \cdots \times T_{X}^{*} \mathcal{B}}_{p \text { copies }} \times \underbrace{T_{X} \mathcal{B} \times \cdots \times T_{X} \mathcal{B}}_{q \text { copies }} \times \underbrace{T_{x}^{*} \mathcal{S} \times \cdots \times T_{x}^{*} \mathcal{S}}_{p \text { copies }} \times \underbrace{T_{x} \mathcal{S} \times \cdots \times T_{x} \mathcal{S}}_{q \text { copies }} \rightarrow \mathrm{R},
$$

where $x=\varphi(X)$.

Let $\mathbf{w}: \mathcal{U} \rightarrow T \mathcal{S}$ be a vector field, where $\mathcal{U} \subset \mathcal{S}$ is open. A curve $\mathbf{c}: I \rightarrow \mathcal{S}$, where $I$ is an open interval, is an integral curve of $\mathbf{w}$ if

$$
\frac{\mathrm{d} \mathbf{c}}{\mathrm{d} t}(r)=\mathbf{w}(\mathbf{c}(r)) \quad \forall r \in I .
$$

If $\mathbf{w}$ depends on time variable explicitly, i.e., $\mathbf{w}: \mathcal{U} \times(-\varepsilon, \varepsilon) \rightarrow T \mathcal{S}$, an integral curve is defined by

$$
\frac{\mathrm{d} \mathbf{c}}{\mathrm{d} t}=\mathbf{w}(\mathbf{c}(t), t)
$$


Let $\mathbf{w}: \mathcal{S} \times I \rightarrow T \mathcal{S}$ be a vector field. The collection of maps $F_{t, s}$ such that for each $s$ and $x$, $t \mapsto F_{t, s}(x)$ is an integral curve of $\mathbf{w}$ and $F_{s, s}(x)=x$ is called the flow of $\mathbf{w}$. Let $\mathbf{w}$ be a $C^{1}$ vector field on $\mathcal{S}, F_{t, s}$ its flow, and $\mathbf{t}$ a $C^{1}$ tensor field on $\mathcal{S}$. The Lie derivative of $\mathbf{t}$ with respect to $\mathbf{w}$ is defined by

$$
\mathbf{L}_{\mathbf{w}} \mathbf{t}=\left.\frac{d}{\mathrm{~d} t}\left(F_{t, s}^{*} \mathbf{t}\right)\right|_{t=s}
$$

If we hold $t$ fixed in $\mathbf{t}$ then we denote

$$
£_{\mathbf{w}} \mathbf{t}=\left.\frac{d}{\mathrm{~d} t}\left(F_{t, s}^{*} \mathbf{t}\right)\right|_{t=s}
$$

which is called the autonomous Lie derivative. Hence

$$
\mathbf{L}_{\mathbf{w}} \mathbf{t}=\frac{\partial}{\partial t} \mathbf{t}+\mathfrak{L}_{\mathbf{w}} \mathbf{t}
$$

Let $\mathbf{v}$ be a vector field on $\mathcal{S}$ and $\varphi: \mathcal{B} \rightarrow \mathcal{S}$ a regular and orientation preserving $C^{1}$ map. The Piola transform of $\mathbf{v}$ is

$$
\mathbf{V}=J \varphi^{*} \mathbf{v}
$$

where $J$ is the Jacobian of $\varphi$. If $\mathbf{Y}$ is the Piola transform of $\mathbf{y}$, then the Piola identity holds,

$$
\operatorname{Div} \mathbf{Y}=J(\operatorname{div} \mathbf{y}) \circ \varphi
$$

A $k$-form on a manifold $M$ is a skew-symmetric $\left(\begin{array}{l}0 \\ k\end{array}\right)$-tensor. The space of $k$-forms on $M$ is denoted $\Omega^{k}(M)$. If $\varphi: M \rightarrow N$ is a regular and orientation preserving $C^{1}$ map and $\alpha \in \Omega^{k}(\varphi(M))$, then

$$
\int_{\varphi(M)} \boldsymbol{\alpha}=\int_{M} \varphi^{*} \boldsymbol{\alpha} .
$$

Geometric continuum mechanics: We next review a few of the basic notions of continuum mechanics from the geometric point of view.

A body $\mathcal{B}$ is a submanifold of a Riemannian manifold $\mathfrak{B}$ and a configuration of $\mathcal{B}$ is a mapping $\varphi: \mathcal{B} \rightarrow \mathcal{S}$, where $\mathcal{S}$ is another Riemannian manifold. The set of all configurations of $\mathcal{B}$ is denoted $\mathcal{C}$. A motion is a curve $c: \mathbb{R} \rightarrow \mathcal{C} ; t \mapsto \varphi_{t}$ in $\mathcal{C}$.

For a fixed $t, \varphi_{t}(X)=\varphi(X, t)$ and for a fixed $X, \varphi_{X}(t)=\varphi(X, t)$, where $X$ is position of material points in the undeformed configuration $\mathcal{B}$. The material velocity is the map $\mathbf{V}_{t}: \mathcal{B} \rightarrow \mathbb{R}^{3}$ given by

$$
\mathbf{V}_{t}(X)=\mathbf{V}(X, t)=\frac{\partial \varphi(X, t)}{\partial t}=\frac{d}{\mathrm{~d} t} \varphi_{X}(t)
$$

Similarly, the material acceleration is defined by

$$
\mathbf{A}_{t}(X)=\mathbf{A}(X, t)=\frac{\partial \mathbf{V}(\mathbf{X}, t)}{\partial t}=\frac{d}{\mathrm{~d} t} \mathbf{V}_{X}(t)
$$

In components

$$
A^{a}=\frac{\partial V^{a}}{\partial t}+\gamma_{b c}^{a} V^{b} V^{c},
$$

where $\gamma_{b c}^{a}$ is the Christoffel symbol of the local coordinate chart $\left\{x^{a}\right\}$. 
Here it is assumed that $\varphi_{t}$ is invertible and regular. The spatial velocity of a regular motion $\varphi_{t}$ is defined as

$$
\mathbf{v}_{t}: \varphi_{t}(\mathcal{B}) \rightarrow \mathbb{R}^{3}, \quad \mathbf{v}_{t}=\mathbf{V}_{t} \circ \varphi_{t}^{-1}
$$

and the spatial acceleration $\mathbf{a}_{t}$ is defined as

$$
\mathbf{a}=\dot{\mathbf{v}}=\frac{\partial \mathbf{v}}{\partial t}+\nabla_{\mathbf{v}} \mathbf{v}
$$

In components

$$
a^{a}=\frac{\partial v^{a}}{\partial t}+\frac{\partial v^{a}}{\partial x^{b}} v^{b}+\gamma_{b c}^{a} v^{b} v^{c}
$$

Let $\varphi: \mathcal{B} \rightarrow \mathcal{S}$ be a $C^{1}$ configuration of $\mathcal{B}$ in $\mathcal{S}$, where $\mathcal{B}$ and $\mathcal{S}$ are manifolds. Recall that the deformation gradient is denoted $\mathbf{F}=T \varphi$. Thus, at each point $X \in \mathcal{B}$, it is a linear map

$$
\mathbf{F}(X): T_{X} \mathcal{B} \rightarrow T_{\varphi(X)} \mathcal{S} .
$$

If $\left\{x^{i}\right\}$ and $\left\{X^{I}\right\}$ are local coordinate charts on $\mathcal{S}$ and $\mathcal{B}$, respectively, the components of $\mathbf{F}$ are

$$
F_{J}^{i}(X)=\frac{\partial \varphi^{i}}{\partial X^{J}}(X) .
$$

The deformation gradient may be viewed as a two-point tensor,

$$
\mathbf{F}(X): T_{x}^{*} \mathcal{S} \times T_{X} \mathcal{B} \rightarrow \mathbb{R} ; \quad(\alpha, \mathbf{V}) \mapsto\left\langle\alpha, T_{X} \varphi \cdot \mathbf{V}\right\rangle .
$$

Suppose $\mathcal{B}$ and $\mathcal{S}$ are Riemannian manifolds with inner products $\langle\langle,\rangle\rangle_{X}$ and $\langle\langle,\rangle\rangle_{x}$ based at $X \in \mathcal{B}$ and $x \in \mathcal{S}$, respectively.

Recall that the transpose of $\mathbf{F}$ is defined by

$$
\mathbf{F}^{\top}: T_{x} \mathcal{S} \rightarrow T_{X} \mathcal{B}, \quad\langle\langle\mathbf{F V}, \mathbf{v}\rangle\rangle_{x}=\left\langle\left\langle\mathbf{V}, \mathbf{F}^{\top} \mathbf{v}\right\rangle\right\rangle_{X}
$$

for all $\mathbf{V} \in T_{X} \mathcal{B}, \mathbf{v} \in T_{x} \mathcal{S}$. In components,

$$
\left(F^{\top}(X)\right)_{i}^{J}=g_{i j}(x) F^{j}{ }_{K}(X) G^{J K}(X),
$$

where $\mathbf{g}$ and $\mathbf{G}$ are metric tensors on $\mathcal{S}$ and $\mathcal{B}$, respectively. On the other hand, the dual of $\mathbf{F}$, a metric independent notion, is defined by

$$
\mathbf{F} *(x): T_{x}^{*} \mathcal{S} \rightarrow T_{X}^{*} \mathcal{B} ; \quad\left\langle\mathbf{F}^{*}(x) \cdot \alpha, \mathbf{W}\right\rangle=\langle\alpha, \mathbf{F}(X) \mathbf{W}\rangle
$$

for all $\alpha \in T_{x}^{*} \mathcal{S}, \mathbf{W} \in T_{X} \mathcal{B}$.

Considering bases $\mathbf{e}_{a}$ and $\mathbf{E}_{A}$ for $\mathcal{S}$ and $\mathcal{B}$, respectively, one can define the corresponding dual bases $\mathbf{e}^{a}$ and $\mathbf{E}^{A}$. The matrix representation of $\mathbf{F}^{*}$ with respect to the dual bases is the transpose of $F_{A}^{a}$. $\mathbf{F}$ and $\mathbf{F}^{*}$ have the following local representations:

$$
\mathbf{F}=F_{K}^{j} \frac{\partial}{\partial x^{j}} \otimes \mathrm{d} X^{K}, \quad \mathbf{F}^{*}=F^{j}{ }_{K} \mathrm{~d} X^{K} \otimes \frac{\partial}{\partial x^{j}} .
$$

The right Cauchy-Green deformation tensor is defined by

$$
\mathbf{C}(X): T_{X} \mathcal{B} \rightarrow T_{X} \mathcal{B}, \quad \mathbf{C}(X)=\mathbf{F}(X)^{T} \mathbf{F}(X) .
$$

In components, 


$$
C_{J}^{I}=\left(F^{T}\right)_{k}^{I} F_{J}^{k}
$$

It is straightforward to show that

$$
\mathbf{C}^{b}=\varphi^{*}(\mathbf{g}), \quad \text { i. e }, C_{I J}=\left(g_{i j} \circ \varphi\right) F_{I}^{i} F_{J}^{j} .
$$

From now on, by $\mathbf{C}$ we mean the tensor with components $C_{I J}$. The Finger tensor is defined as $\mathbf{b}=\varphi_{t^{*}} \mathbf{G}$, where $\mathbf{G}$ is the metric of the reference configuration.

To make ideas more concrete, a comment is in order. In the geometric treatment of continuum mechanics one assumes that the material body is a Riemannian manifold $(\mathcal{B}, \mathbf{G})$. Here $\mathcal{B}$ is an embedding of the material body, i.e., material points are identified with their positions in the reference configuration. A deformation of the material body is represented by a mapping $\varphi: \mathcal{B}$ $\rightarrow \mathcal{S}$, where $(\mathcal{S}, \mathbf{g})$ is the ambient space, which is another Riemannian manifold. If $\varphi=\mathrm{Id}$, the reference configuration is a trivial embedding of the material body in the ambient space. Physically, in the deformation process the relative distance of material points change in general. In other words, in terms of material points $\mathbf{X}, \mathbf{X}+\mathrm{d} \mathbf{X}$ and their positions in the deformed configuration $\mathbf{x}, \mathbf{x}+\mathrm{d} \mathbf{x}$ we have

$$
\mathrm{d} \mathbf{x} \cdot \mathrm{d} \mathbf{x}=\mathbf{C} \mathrm{d} \mathbf{X} \cdot \mathrm{d} \mathbf{X} \neq \mathrm{d} \mathbf{X} \cdot \mathrm{d} \mathbf{X} .
$$

This means that in general

$$
\mathbf{g} \neq \varphi_{t^{*}} \mathbf{G} .
$$

The following identities will be used frequently in this paper.

$$
\begin{gathered}
\frac{\partial g_{a b}}{\partial x^{c}}=g_{a d} \gamma_{b c}^{d}+g_{b d} \gamma_{a c}^{d}, \\
\frac{\partial G_{A B}}{\partial X^{C}}=G_{A D} \Gamma_{B C}^{D}+G_{B D} \Gamma_{A C}^{D},
\end{gathered}
$$

where $\gamma_{b c}^{d}$ and $\Gamma_{B C}^{D}$ are the Christoffel symbols associated to the metric tensors $\mathbf{g}$ and $\mathbf{G}$, respectively. The covariant derivative of two-point tensors will also be used frequently in this paper. The following two examples would be useful to clarify the idea. For definition for an arbitrary twopoint tensor the reader may refer to Marsden and Hughes, ${ }^{28}$

$$
\begin{gathered}
P_{\mid B}^{a A}=\frac{\partial P^{a A}}{\partial X^{B}}+P^{a C} \Gamma_{C B}^{A}+P^{b A} F_{A}^{c} \gamma_{b c}^{a}, \\
Q_{a}{ }_{\mid B}=\frac{\partial Q_{a}{ }^{A}}{\partial X^{B}}+Q_{a}{ }^{C} \Gamma_{C B}^{A}-Q_{b}{ }^{A} F_{A}^{c} \gamma_{c a}^{b} .
\end{gathered}
$$

Let $\varphi_{t}: \mathcal{B} \rightarrow \mathcal{S}$ be a regular motion of $\mathcal{B}$ in $\mathcal{S}$ and $\mathcal{P} \subset \mathcal{B}$ a $k$-dimensional submanifold. The transport theorem says that for any $k$-form $\alpha$ on $\mathcal{S}$,

$$
\frac{d}{\mathrm{~d} t} \int_{\varphi_{t}(\mathcal{P})} \alpha=\int_{\varphi_{t}(\mathcal{P})} \mathbf{L}_{\mathbf{v}} \alpha
$$

where $\mathbf{v}$ is the spatial velocity of the motion. In a special case when $\alpha=f \mathrm{~d} v$ and $\mathcal{P}=\mathcal{U}$ is an open set,

$$
\frac{d}{\mathrm{~d} t} \int_{\varphi_{t}(\mathcal{P})} f \mathrm{~d} v=\int_{\varphi_{t}(\mathcal{P})}\left[\frac{\partial f}{\partial t}+\operatorname{div}(f \mathbf{v})\right] \mathrm{d} v .
$$

We say that a body $\mathcal{B}$ satisfies balance of linear momentum if for every nice open set $\mathcal{U} \subset \mathcal{B}$, 


$$
\frac{d}{\mathrm{~d} t} \int_{\varphi_{t}(\mathcal{U})} \rho \mathbf{v} \mathrm{d} v=\int_{\varphi_{t}(\mathcal{U})} \rho \mathbf{b} \mathrm{d} v+\int_{\partial \varphi_{t}(\mathcal{U})} \mathbf{t} \mathrm{d} a,
$$

where $\rho=\rho(\mathbf{x}, t)$ is mass density, $\mathbf{b}=\mathbf{b}(\mathbf{x}, t)$ is body force vector field and $\mathbf{t}=\mathbf{t}(\mathbf{x}, \hat{\mathbf{n}}, t)$ is the traction vector. Note that Cauchy's stress theorem tells us that there is a contravariant second-order tensor $\boldsymbol{\sigma}=\boldsymbol{\sigma}(\mathbf{x}, t)$ (Cauchy stress tensor) with components $\sigma^{i j}$ such that $\mathbf{t}=\langle\langle\boldsymbol{\sigma}, \hat{\mathbf{n}}\rangle\rangle$. Note that $\langle\langle\rangle$,$\rangle is the$ inner product induced by the Riemmanian metric $\mathbf{g}$. Equivalently, balance of linear momentum can be written in the undeformed configuration as

$$
\frac{d}{\mathrm{~d} t} \int_{\mathcal{U}} \rho_{0} \mathbf{V} \mathrm{d} V=\int_{\mathcal{U}} \rho_{0} \mathbf{B} \mathrm{d} V+\int_{\partial \mathcal{U}}\langle\langle\mathbf{P}, \hat{\mathbf{N}}\rangle\rangle \mathrm{d} A,
$$

where, $\mathbf{P}=J \varphi^{*} \boldsymbol{\sigma}$ (the first Piola-Kirchhoff stress tensor) is the Piola transform of Cauchy stress tensor. Note that $\mathbf{P}$ is a two-point tensor with components $P^{i J}$. Note also that this is the balance of linear momentum in the deformed (physical) space written in terms of some quantities that are defined with respect to the reference configuration.

As was mentioned before, balance of linear momentum has no intrinsic meaning because integrating a vector field is geometrically meaningless. As is standard in continuum mechanics, this balance law makes use of the linear (or affine) structure of Euclidean space.

A body $\mathcal{B}$ is said to satisfy balance of angular momentum if for every nice open set $\mathcal{U} \subset \mathcal{B}$,

$$
\frac{d}{\mathrm{~d} t} \int_{\varphi_{t}(\mathcal{U})} \rho \mathbf{x} \times \mathbf{v} \mathrm{d} v=\int_{\varphi_{t}(\mathcal{U})} \rho \mathbf{x} \times \mathbf{b} \mathrm{d} v+\int_{\partial \varphi_{t}(\mathcal{U})} \mathbf{x} \times\langle\langle\boldsymbol{\sigma}, \hat{\mathbf{n}}\rangle\rangle \mathrm{d} a .
$$

As with balance of linear momentum, balance of angular momentum makes use of the linear structure of Euclidean space and this does not transform in a covariant way under a general change of coordinates.

One says that balance of energy holds if, for every nice open set $\mathcal{U} \subset \mathcal{B}$,

$$
\frac{d}{\mathrm{~d} t} \int_{\varphi_{t}(\mathcal{U})} \rho\left(e+\frac{1}{2}\langle\langle\mathbf{v}, \mathbf{v}\rangle\rangle\right) \mathrm{d} v=\int_{\varphi_{t}(\mathcal{U})} \rho(\langle\langle\mathbf{b}, \mathbf{v}\rangle\rangle+r) \mathrm{d} v+\int_{\partial \varphi_{t}(\mathcal{U})}(\langle\langle\mathbf{t}, \mathbf{v}\rangle\rangle+h) \mathrm{d} a,
$$

where $e=e(\mathbf{x}, t), r=r(\mathbf{x}, t)$ and $h=h(\mathbf{x}, \hat{\mathbf{n}}, t)$ are internal energy per unit mass, heat supply per unit mass and heat flux, respectively.

The geometry of inverse motions: The study of inverse motions in continuum mechanics was started by Shield ${ }^{38}$ and further extended by Ericksen ${ }^{10}$ and Steinmann. ${ }^{43,42}$ Here the idea is to fix spatial points and look at the evolution of material points under the inverse of the deformation mapping. It is known that in inverse motion, Eshelby's tensor has a role similar to that of stress tensor in direct motion. One should note that formulating continuum mechanics in terms of the inverse motion is simply a change in describing the same physical system and so, in general, cannot have any profound consequences. However, in the general relativistic setting, in which it is desireable to have the fields to be defined on space-time and take values in a bundle over spacetime, inverse configurations are preferred; see Ref. 5 and references therein.

\section{LAGRANGIAN FIELD THEORY OF ELASTIC BODIES WITH EVOLVING REFERENCE CONFIGURATIONS}

Suppose the reference configuration evolves in time and assume that this evolution can be represented by a one-parameter family of mappings that map $\mathcal{B} \subset \mathfrak{B}$ (reference configuration at $t=0)$ to $\mathcal{B}_{t} \subset \mathfrak{B}$ (the reference configuration at time $t$ ),

$$
\Xi_{t}: \mathcal{B} \rightarrow \mathcal{B}_{t}
$$

We call these maps the configurational deformation maps. Note that this is not the most general form of reference configuration evolution. In general, one should look at the reference configura- 


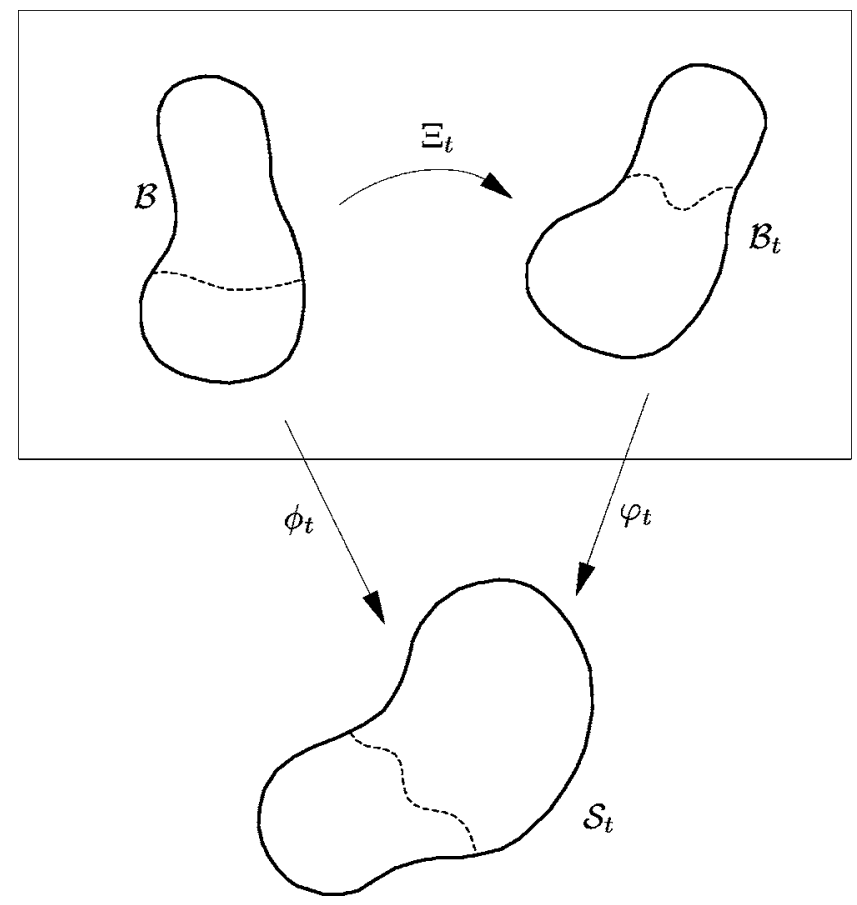

FIG. 1. Configurational and standard deformation maps.

tion evolution locally (see Refs. 9 and 8 for some discussions on this). For the sake of simplicity, we assume a global reference configuration evolution. The configuration space for the evolution of the reference configuration is

$$
\mathcal{C}^{\mathrm{conf}}=\left\{\Xi \mid \Xi: \mathcal{B} \rightarrow \mathcal{B}_{t}\right\}
$$

An evolution of the reference configuration is a curve $c: I \rightarrow \mathcal{C}^{\text {conf }}$ in $\mathcal{C}^{\text {conf }}$. It is important to put the right restrictions on $\Xi_{t}$. It does not seem necessary for $\Xi_{t}$ to be invertible, in general. Here, we assume that $\Xi_{t}$ is a diffeomorphism. A standard deformation is represented by a one-parameter family of mappings,

$$
\varphi_{t}: \mathcal{B}_{t} \rightarrow \mathcal{S} .
$$

The standard configuration space is defined by

$$
\mathcal{C}=\left\{\varphi \mid \varphi: \mathcal{B}_{t} \rightarrow \mathcal{S}\right\} .
$$

Again, a standard deformation is a curve in the standard configuration space. The total deformation map is the composition of standard and configurational deformation maps,

$$
\phi_{t}=\varphi_{t}^{\circ} \Xi_{t}: \mathcal{B} \rightarrow \mathcal{S} ;
$$

that is, the following diagram commutes:

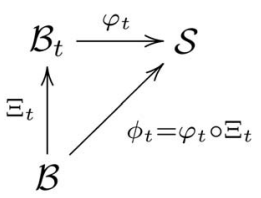

Figure 1 below shows the same idea schematically. 
In terms of mapping the material points, $x_{t}=\varphi_{t}\left(X_{t}\right)=\varphi_{t}{ }^{\circ} \Xi_{t}(X)$, as is shown in the following commutative diagram:

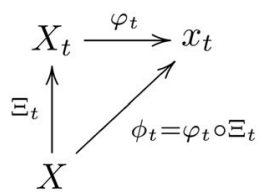

The configuration space for the total deformation is defined as

$$
\mathcal{C}^{\mathrm{tot}}=\left\{\phi \mid \phi=\varphi \circ \Xi, \varphi \in \mathcal{C}, \Xi \in \mathcal{C}^{\mathrm{conf}}\right\}=\mathcal{C} \circ \mathcal{C}^{\mathrm{conf}}
$$

A deformation is a curve $c: I \rightarrow \mathcal{C}^{\text {tot }}$ in the total configuration space. Note that $\Xi_{t}=$ Id (identity map) in most of classical continuum mechanics.

Notice that there are two independent deformation mappings $\varphi_{t}$ and $\Xi_{t}$ when reference configuration evolves in time (see Fig. 1). These separate mappings represent independent kinematical processes and hence may correspond to two separate systems of forces, in general.

Definition 3.1 (configurational velocity): The configurational velocity is defined by

$$
\mathbf{V}_{0}(X, t)=\frac{\partial \Xi_{t}(X)}{\partial t}
$$

Definition 3.2: The total material velocity is defined by

$$
\tilde{\mathbf{V}}(\mathbf{X}, t)=\left.\frac{\partial \phi_{t}\left(\mathbf{X}_{t}\right)}{\partial t}\right|_{\mathbf{X} \text { fixed }}=\frac{\partial \varphi_{t}}{\partial t}+\mathbf{F} \mathbf{V}_{0}=\mathbf{V}+\mathbf{F} \mathbf{V}_{0}
$$

where, as before, $\mathbf{F}=\partial \varphi_{t} / \partial \mathbf{X}_{t}$ is the deformation gradient (holding $t$ fixed). Note that

$$
T \phi_{t}=T \varphi_{t} \circ T \Xi_{t} \quad \text { or } \quad \widetilde{\mathbf{F}}=\mathbf{F F}_{0} .
$$

Thus

$$
\mathbf{F}_{0}=\mathbf{F}^{-1} \circ \tilde{\mathbf{F}} .
$$

Now we may think about postulating the conservation of configurational mass and balance of linear and angular configurational momenta.

Conservation of mass is defined in terms of conservation of mass for deformation mappings $\Xi_{t}$ and $\varphi_{t}$ separately or equivalently for $\Xi_{t}$ and $\phi_{t}$ separately. This makes sense as $\Xi_{t}$ and $\varphi_{t}$ correspond to configurational and standard deformations and should preserve the mass of an arbitrary sub-body.

Definition 3.3 (conservation of mass): Suppose $\mathcal{B}$ is a body and $\phi_{t}=\varphi_{t}^{\circ} \Xi_{t}$ is a deformation map. We say that the deformation mapping is mass conserving if for every $\mathcal{U} \subset \mathcal{B}$,

$$
\frac{d}{\mathrm{~d} t} \int_{\Xi_{t}(\mathcal{U})} \rho_{0}\left(\mathbf{X}_{t}, t\right) \mathrm{d} V=0 \quad \text { and } \quad \frac{d}{\mathrm{~d} t} \int_{\phi_{t}(\mathcal{U})} \rho(\mathbf{x}, t) \mathrm{d} v=0,
$$

where $\rho_{0}\left(\mathbf{X}_{t}, t\right)$ is the mass density at point $X_{t} \in \mathcal{B}_{t}$ and $\rho(\mathbf{x}, t)$ is the mass density at the point $\mathbf{x}$ $\in \mathcal{S}$.

Localization of the above equations gives the local form of conservation of mass, namely

$$
R_{0}(\mathbf{X})=\rho_{0}\left(\mathbf{X}_{t}, t\right) J_{0}=\rho(\mathbf{x}, t) \tilde{J},
$$

where $J_{0}=\operatorname{det}\left(\mathbf{F}_{0}\right)\left(\sqrt{\operatorname{det} \mathbf{G}} / \sqrt{\operatorname{det} \mathbf{G}_{0}}\right), \mathbf{F}_{0}=T \Xi_{t}$ is the configurational deformation gradient, $\mathbf{G}_{0}$ is the fixed metric of $\mathcal{B}, \mathbf{G}$ is the metric of $\mathcal{B}_{t}$ and $R_{0}$ is the mass density at $\mathbf{X} \in \mathcal{B}$ and $\widetilde{J}=\operatorname{det}(\widetilde{\mathbf{F}})$ $\times(\sqrt{\operatorname{det} \mathbf{g}} / \sqrt{\operatorname{det} \mathbf{G}})=J J_{0}$. Note that this is equivalent to 


$$
R_{0}=\rho_{0} J_{0} \quad \text { and } \quad \rho_{0}=\rho J .
$$

One may be tempted to postulate a balance of configurational linear momentum as follows. A body $\mathcal{B}$ satisfies the balance of configurational linear momentum if for any $\mathcal{U}^{\prime} \subset \mathcal{B}_{t}$,

$$
\frac{d}{\mathrm{~d} t} \int_{\mathcal{U}^{\prime}} \rho_{0} \mathbf{V}_{0} \mathrm{~d} V=\int_{\mathcal{U}^{\prime}} \rho_{0} \mathbf{B}_{0} \mathrm{~d} V+\int_{\partial \mathcal{U}^{\prime}} \mathbf{P}_{0} \mathbf{N} \mathrm{d} A
$$

Localization of this balance law and using Cauchy's theorem gives the following local form of the balance of configurational linear momentum

$$
\operatorname{Div} \mathbf{P}_{0}+\rho_{0} \mathbf{B}_{0}=\rho_{0} \mathbf{A}_{0} .
$$

Thinking of configurational deformation mapping $\Xi_{t}$ as a deformation of a fixed reference configuration, this balance law is similar to the standard balance of linear momentum written in the deformed configuration. Note that postulating such a balance law requires the introduction of two new quantities, namely $\mathbf{P}_{0}$ and $\mathbf{B}_{0}$ and does not seem to be of any use at this point.

It should be noted that a configurational change need not be volume preserving. An example is a phase transformation from cubic to tetragonal which has the following configurational deformation gradient (this is called Bain strain or matrix in martensitic phase transformations),

$$
\mathbf{F}_{0}=\left(\begin{array}{ccc}
1 & 0 & 0 \\
0 & 1 & 0 \\
0 & 0 & \frac{c}{a}
\end{array}\right)
$$

where $a=b$ and $c>a$ are the tetragonal lattice parameters.

The Lagrangian may be regarded as a map $L: T C \rightarrow R$, where $\mathcal{C}$ is the space of some sections (for technical details see Ref. 28), associated to the Lagrangian density $\mathcal{L}$ and a volume element $\mathrm{d} V(X)$ on $\mathcal{B}$ and is defined as

$$
L(\varphi, \dot{\varphi})=\int_{\mathcal{B}} \mathcal{L}(X, \varphi(X), \dot{\varphi}(X), \mathbf{F}(X), \mathbf{G}(X), \mathbf{g}(\varphi(X))) \mathrm{d} V(X) .
$$

Note that here we have assumed an explicit dependence of $\mathcal{L}$ on the material and spatial metrics G and $\mathbf{g}$. Let us first revisit the classical Lagrangian field theory of elasticity using the above Lagrangian density with explicit dependence on material and spatial metrics. The action function is defined as

$$
S(\varphi)=\int_{t_{0}}^{t_{1}} L(\varphi, \dot{\varphi}) \mathrm{d} t
$$

Hamilton's principle states that the physical configuration $\varphi$ is the critical point of the action, i.e.,

$$
\mathbf{d} S(\varphi) \cdot \delta \varphi=0
$$

Note that variation in $\varphi$ leaves the material metric unchanged. The statement of Hamilton's principle can be simplified to read

$$
\int_{t_{0}}^{t_{1}} \int_{\mathcal{B}}\left(\frac{\partial \mathcal{L}}{\partial \varphi} \cdot \delta \varphi+\frac{\partial \mathcal{L}}{\partial \dot{\varphi}} \cdot \delta \dot{\varphi}+\frac{\partial \mathcal{L}}{\partial \mathbf{F}}: \delta \mathbf{F}+\frac{\partial \mathcal{L}}{\partial \mathbf{g}}: \delta \mathbf{g}\right) \mathrm{d} V(X) \mathrm{d} t=0
$$

After some manipulations the above integral statement results in 


$$
\frac{\partial \mathcal{L}}{\partial \varphi^{a}}-\frac{\partial}{\partial t}\left(\frac{\partial \mathcal{L}}{\partial \dot{\varphi}}\right)_{a}-\left(\frac{\partial \mathcal{L}}{\partial \mathbf{F}}\right)_{a \mid A}^{A}-\left(\frac{\partial \mathcal{L}}{\partial \mathbf{F}}\right)_{b}^{A} F_{A}^{c} \gamma_{a c}^{b}+2 \frac{\partial \mathcal{L}}{\partial g_{c d}} g_{b d} \gamma_{a c}^{b}=0
$$

Noting that

$$
\begin{gathered}
\frac{d}{\mathrm{~d} t}\left(\frac{\partial \mathcal{L}}{\partial \dot{\varphi}}\right)_{a}=\rho_{0}\left(g_{a b} A^{b}+g_{b c} \gamma_{a d}^{c} \dot{\varphi}^{b} \dot{\varphi}^{d}\right) \\
\left(\frac{\partial \mathcal{L}}{\partial \mathbf{F}}\right)_{a}^{A}=-P_{a}{ }^{A} \\
2 \frac{\partial \mathcal{L}}{\partial g_{c d}}=\rho_{0} \dot{\varphi}^{c} \dot{\varphi}^{d}-J \sigma^{c d}
\end{gathered}
$$

Eq. (3.21) can be written as

$$
P_{a \mid A}^{A}+\frac{\partial \mathcal{L}}{\partial \varphi^{a}}+\left(F^{c}{ }_{A} P_{b}^{A}-J \sigma^{c d} g_{b d}\right) \gamma_{a c}^{b}=\rho_{0} g_{a b} A^{b} .
$$

Note that if $\mathcal{L}$ depends on $\mathbf{F}$ and $\mathbf{g}$ through $\mathbf{C}$, then the term in the parentheses would be zero and hence

$$
\left.P_{a}^{A}\right|_{A}+\frac{\partial \mathcal{L}}{\partial \varphi^{a}}=\rho_{0} g_{a b} A^{b},
$$

which is nothing but the familiar equations of motion. [Also note that in (3.25) use was made of Doyle-Ericksen formula (3.24). However, for arriving at (3.26) there is no need for using DoyleEricksen formula.]

Now suppose that during the process of deformation the continuum undergoes a continuous material evolution. This means that the deformation mapping $\varphi$ is the composition of a total deformation mapping and a referential mapping, i.e.,

$$
\varphi=\phi \circ \Xi^{-1} \text { or } \phi=\varphi \circ \Xi .
$$

Note that defining such a composition is ambiguous because there are infinitely many possibilities for decomposing a given deformation mapping $\phi$ into two mappings $\varphi$ and $\Xi$. The new mappings can represent part of the standard deformation and material evolution. To make sure that $\varphi$ is the standard part of total deformation mapping, the Lagrangian is written as an integral on the current reference configuration $\mathcal{B}_{t}$

$$
L(\varphi, \dot{\varphi})=\int_{\mathcal{B}_{t}} \mathcal{L}(X, \varphi(X), \dot{\varphi}(X), \mathbf{F}(X), \mathbf{G}(X), \mathbf{g}(\varphi(X))) \mathrm{d} V(X) .
$$

It would be more convenient to write the Lagrangian as a functional on $\mathcal{B}$ (the fixed initial reference configuration). Let us denote points on $\mathcal{B}$ by $U$. Note that

$$
\dot{\phi}(U)=(\dot{\varphi} \circ \Xi)(U)+T \varphi(\Xi(U)) \cdot \dot{\Xi}(U) \quad \text { or } \quad(\dot{\varphi} \circ \Xi)(U)=\dot{\phi}(U)-\mathbf{F}(\Xi(U)) \cdot \dot{\Xi}(U) \text {. }
$$

Also

$$
\mathbf{F}(\Xi(U))=\mathbf{F}_{\phi}(U) \mathbf{F}_{\Xi}^{-1}(\Xi(U)) .
$$

Thus, 


$$
\mathcal{L}=\mathcal{L}\left(\Xi(U), \phi(U), \dot{\phi}(U)-\mathbf{F}_{\phi}(U) \mathbf{F}_{\Xi}^{-1}(\Xi(U)) \cdot \dot{\Xi}(U), \mathbf{F}_{\phi}(U) \mathbf{F}_{\Xi}^{-1}(\Xi(U)), \mathbf{G}(\Xi(U)), \mathbf{g}(\phi(U))\right) J_{\Xi}(U),
$$

where

$$
J_{\Xi}=\operatorname{det}(T \Xi) \frac{\sqrt{\operatorname{det} \mathbf{G}}}{\sqrt{\operatorname{det} \mathbf{G}_{0}}},
$$

and where $\mathbf{G}_{0}$ is the fixed metric of the fixed reference configuration and $\mathbf{G}$ is the metric of $\mathcal{B}_{t}$. As before, the action is defined as

$$
S(\Xi, \phi)=\int_{t_{0}}^{t_{1}} L(\Xi, \dot{\Xi}, \phi, \dot{\phi}) \mathrm{d} t
$$

Hamilton's principle states that the physical configurations $\Xi$ and $\phi$ are the critical points of the action, i.e.,

$$
\mathbf{d} S(\Xi, \phi) \cdot(\delta \Xi, \delta \phi)=0 .
$$

For the sake of clarity, we look at the two independent variations separately.

\section{A. Vertical variations}

Let us first look at vertical variations; that is, we assume that $\delta \Xi=0$ and see if we can recover the classical Euler-Lagrange equations.

Proposition 3.4: Allowing only vertical variations in Hamilton's principle, one obtains the following equations of motion

$$
\frac{\partial \mathcal{L}}{\partial \varphi^{a}}-\frac{d}{\mathrm{~d} t}\left(\frac{\partial \mathcal{L}}{\partial \dot{\varphi} \circ \Xi}\right)_{a}-\left(\frac{\partial \mathcal{L}}{\partial \mathbf{F}}\right)_{a \mid B}^{B}-F_{B}^{c} \gamma_{a c}^{b}\left(\frac{\partial \mathcal{L}}{\partial \mathbf{F}}\right)_{b}^{B}+\frac{\partial \mathcal{L}}{\partial g^{b c}} \frac{\partial g^{b c}}{\partial x^{a}}=0
$$

Proof: The derivative of the action with respect to vertical variations is computed as follows:

$$
\begin{aligned}
\mathbf{d} S(\Xi, \phi) \cdot(0, \delta \phi)= & \int_{t_{0}}^{t_{1}} \int_{\mathcal{B}}\left\{\frac{\partial \mathcal{L}}{\partial \varphi \circ \Xi} \cdot \delta \phi+\frac{\partial \mathcal{L}}{\partial \dot{\varphi} \circ \Xi} \cdot\left(\delta \dot{\phi}-\delta\left[\mathbf{F}_{\phi}(U) \mathbf{F}_{\Xi}^{-1}(\Xi(U)) \cdot \dot{\Xi}(U)\right]\right)\right. \\
& \left.+\frac{\partial \mathcal{L}}{\partial \mathbf{F}}: \delta\left[\mathbf{F}_{\phi}(U) \mathbf{F}_{\Xi}^{-1}(\Xi(U))\right]+\frac{\partial \mathcal{L}}{\partial \mathbf{g}}: \delta \mathbf{g} \circ \phi\right\} J_{\Xi}(U) \mathrm{d} V(U) \mathrm{d} t=0
\end{aligned}
$$

Note that

$$
\begin{aligned}
\delta\left(\mathbf{F}_{\phi} \mathbf{F}_{\Xi}^{-1} \circ \Xi\right) & =\delta\left(\mathbf{F}_{\phi} \mathbf{F}_{\Xi}^{-1}\right) \circ \Xi=T\left(\delta\left(\phi \circ \Xi^{-1}\right)\right) \circ \Xi=T\left(\delta \phi \circ \Xi^{-1}\right) \circ \Xi \\
& =\left(T \delta \phi T \Xi^{-1}\right) \circ \Xi=D \delta \phi \mathbf{F}_{\Xi}^{-1} \circ \Xi .
\end{aligned}
$$

Let us assume coordinates $\left\{U^{\alpha}\right\},\left\{X^{A}\right\}$, and $\left\{x^{a}\right\}$ and basis vectors $\mathbf{E}_{\alpha}, \mathbf{e}_{A}$, and $\mathbf{f}_{a}$ on $\mathcal{B}, \mathcal{B}_{t}$, and $\mathcal{S}$, respectively. Thus, in coordinates

$$
D \delta \phi=\frac{\partial \delta \phi^{a}}{\partial U^{\alpha}} \mathbf{f}_{a} \otimes \mathbf{E}^{\alpha} .
$$

The first part of the second term is simplified as 


$$
\int_{t_{0}}^{t_{1}} \int_{\mathcal{B}} \frac{\partial \mathcal{L}}{\partial \dot{\varphi} \circ \Xi} \delta \dot{\phi} J_{\Xi}(U) \mathrm{d} V(U)=-\int_{t_{0}}^{t_{1}} \int_{\mathcal{B}}\left[\frac{d}{\mathrm{~d} t}\left(\frac{\partial \mathcal{L}}{\partial \dot{\varphi} \circ \Xi}\right)_{a}+\frac{\partial \mathcal{L}}{\partial \dot{\varphi} \circ \Xi^{a}} W^{B}{ }_{\mid B}\right] \delta \phi^{a} J_{\Xi} \mathrm{d} V(U) \mathrm{d} t,
$$

where

$$
\mathbf{W}(U)=\frac{d}{\mathrm{~d} t} \Xi(U) .
$$

The second part of the second term in (3.36) can be simplified to

$$
\begin{aligned}
-\int_{t_{0}}^{t_{1}} & \int_{\mathcal{B}} J_{\Xi} \frac{\partial \mathcal{L}}{\partial \dot{\varphi} \circ \Xi} D \delta \phi \mathbf{F}_{\Xi}^{-1} \circ \Xi \cdot \mathbf{W} \mathrm{d} V(U) \mathrm{d} t \\
= & \int_{t_{0}}^{t_{1}} \int_{\mathcal{B}}\left[\left(\frac{\partial \mathcal{L}}{\partial \dot{\varphi} \circ \Xi}\right)_{a} J_{\Xi}\left(\mathbf{F}_{\Xi}^{-1} \circ \Xi\right)^{\beta}{ }_{B} W^{B}\right]_{\mid \beta} \delta \phi^{a} \mathrm{~d} V(U) \mathrm{d} t \\
& \quad+\int_{t_{0}}^{t_{1}} \int_{\mathcal{B}}\left[J_{\Xi}\left(\frac{\partial \mathcal{L}}{\partial \dot{\varphi} \circ \Xi}\right)_{b}(\mathbf{F} \circ \Xi)^{c}{ }_{A} \gamma_{a c}^{b} W^{A}\right] \delta \phi^{a} \mathrm{~d} V(U) \mathrm{d} t .
\end{aligned}
$$

Using the Piola identity we have

$$
\left[\left(\frac{\partial \mathcal{L}}{\partial \dot{\varphi} \circ \Xi}\right)_{a} J_{\Xi}\left(\mathbf{F}_{\Xi}^{-1} \circ \Xi\right)^{\beta}{ }_{B} W^{B}\right]_{\mid \beta}=J_{\Xi}\left[\left(\frac{\partial \mathcal{L}}{\partial \dot{\varphi} \circ \Xi}\right)_{a} W^{A}\right]_{\mid A} .
$$

Also

$$
\left[\left(\frac{\partial \mathcal{L}}{\partial \dot{\varphi} \circ \Xi}\right)_{a} W^{A}\right]_{\mid A}=\frac{\partial}{\partial X^{A}}\left(\frac{\partial \mathcal{L}}{\partial \dot{\varphi} \circ \Xi}\right)_{a} W^{A}+\left(\frac{\partial \mathcal{L}}{\partial \dot{\varphi} \circ \Xi}\right)_{a} W_{\mid A}^{A}-\left(\frac{\partial \mathcal{L}}{\partial \dot{\varphi} \circ \Xi}\right)_{b} W^{A} \gamma_{a c}^{b} F_{A}^{c} .
$$

Therefore (3.41) is simplified to

$$
\int_{t_{0}}^{t_{1}} \int_{\mathcal{B}}\left[\frac{\partial}{\partial X^{A}}\left(\frac{\partial \mathcal{L}}{\partial \dot{\varphi} \circ \Xi}\right)_{a} W^{A}+\left(\frac{\partial \mathcal{L}}{\partial \dot{\varphi} \circ \Xi}\right)_{a} W^{A}{ }_{\mid A}\right] \delta \phi^{a} J_{\Xi} \mathrm{d} V(U) \mathrm{d} t .
$$

Note that

$$
\frac{\partial}{\partial t}\left(\frac{\partial \mathcal{L}}{\partial \dot{\varphi}}\right)_{a}=\frac{\partial}{\partial t}\left(\frac{\partial \mathcal{L}}{\partial \dot{\varphi} \circ \Xi}\right)_{a} \circ \Xi^{-1}-\frac{\partial}{\partial X^{A}}\left(\frac{\partial \mathcal{L}}{\partial \dot{\varphi} \circ \Xi}\right)_{a} \circ \Xi^{-1} W^{A} .
$$

Hence adding (3.39) and (3.44) the term corresponding to $\delta \dot{\phi}$ is simplified to

$$
\int_{t_{0}}^{t_{1}} \int_{\mathcal{B}_{t}}-\frac{\partial}{\partial t}\left(\frac{\partial \mathcal{L}}{\partial \dot{\varphi}}\right)_{a} \delta \phi^{a} \circ \Xi^{-1} \mathrm{~d} V(X) \mathrm{d} t .
$$

After some lengthy manipulations, the third term in (3.36) can be written as

$$
-\int_{t_{0}}^{t_{1}} \int_{\mathcal{B}}\left[\left(\frac{\partial \mathcal{L}}{\partial \mathbf{F} \circ \Xi}\right)_{a \mid B}^{B}+F_{B}^{c} \circ \Xi\left(\frac{\partial \mathcal{L}}{\partial \mathbf{F} \circ \Xi}\right)_{a}^{B} \gamma_{a c}^{b}\right] \delta \phi^{a} J_{\Xi}(U) \mathrm{d} V(U) \mathrm{d} t .
$$

The last term is simplified as 


$$
\begin{gathered}
\int_{t_{0}}^{t_{1}} \int_{\mathcal{B}} \frac{\partial \mathcal{L}}{\partial \mathbf{g} \circ \phi}: \delta \mathbf{g} \circ \phi J_{\Xi} \mathrm{d} V(U) \mathrm{d} t=\int_{t_{0}}^{t_{1}} \int_{\mathcal{B}} \frac{\partial \mathcal{L}}{\partial g^{b c} \circ \phi} \frac{\partial g^{b c}}{\partial x^{a}} \delta \phi^{a} J_{\Xi} \mathrm{d} V(U) \mathrm{d} t=\int_{t_{0}}^{t_{1}} \int_{\mathcal{B}_{t}} \frac{\partial \mathcal{L}}{\partial g^{b c}} \frac{\partial g^{b c}}{\partial x^{a}} \delta \phi^{a} \\
\circ \Xi^{-1} \mathrm{~d} V(X) \mathrm{d} t=-\int_{t_{0}}^{t_{1}} \int_{\mathcal{B}_{t}} \frac{\partial \mathcal{L}}{\partial g^{b c}}\left(g^{c d} \gamma_{a d}^{b}+g^{b d} \gamma_{a d}^{c}\right) \delta \phi^{a} \circ \Xi^{-1} \mathrm{~d} V(X) \mathrm{d} t .
\end{gathered}
$$

Therefore, adding the above four simplified terms, we obtain

$$
\begin{aligned}
\mathbf{d} S(\Xi, \phi) \cdot(0, \delta \phi) & =\int_{t_{0}}^{t_{1}} \int_{\mathcal{B}_{t}}\left[\frac{\partial \mathcal{L}}{\partial \varphi^{a}}-\frac{d}{\mathrm{~d} t}\left(\frac{\partial \mathcal{L}}{\partial \dot{\varphi}}\right)_{a}-\left(\frac{\partial \mathcal{L}}{\partial \mathbf{F}}\right)_{a \mid B}^{B}-F_{B}^{c} \gamma_{a c}^{b}\left(\frac{\partial \mathcal{L}}{\partial \mathbf{F}}\right)_{b}^{B}+\frac{\partial \mathcal{L}}{\partial g^{b c}} \frac{\partial g^{b c}}{\partial x^{a}}\right] \delta \phi^{a} \\
& \circ \Xi^{-1} \mathrm{~d} V(X) \mathrm{d} t .
\end{aligned}
$$

As $\delta \phi^{a}$ is arbitrary we conclude that

$$
\frac{\partial \mathcal{L}}{\partial \varphi^{a}}-\frac{d}{\mathrm{~d} t}\left(\frac{\partial \mathcal{L}}{\partial \dot{\varphi} \circ \Xi}\right)_{a}-\left(\frac{\partial \mathcal{L}}{\partial \mathbf{F}}\right)_{a \mid B}^{B}-F_{B}^{c} \gamma_{a c}^{b}\left(\frac{\partial \mathcal{L}}{\partial \mathbf{F}}\right)_{b}^{B}+\frac{\partial \mathcal{L}}{\partial g^{b c}} \frac{\partial g^{b c}}{\partial x^{a}}=0,
$$

which gives the stated result.

\section{B. Horizontal variations}

Now let us try to find the Euler-Lagrange equations resulting from horizontal variations; that is, variations of the configurational deformation mapping $\Xi$.

Proposition 3.5: Allowing only horizontal variations in Hamilton's principle, one obtains the following configurational equations of motion:

$$
\frac{\partial \mathcal{L}}{\partial X^{A}}+\frac{\partial}{\partial t}\left[\left(\frac{\partial \mathcal{L}}{\partial \dot{\varphi}}\right)_{a} F_{A}^{a}\right]-\left[\mathcal{L} \delta^{B}{ }_{A}-\left(\frac{\partial \mathcal{L}}{\partial \mathbf{F}}\right)_{a}^{B} F^{a}{ }_{A}\right]_{\mid B}+\left(\frac{\partial \mathcal{L}}{\partial \mathbf{F}}\right)_{a}^{B} F_{C}^{a} \Gamma_{A B}^{C}+2 G_{C D} \Gamma_{A B}^{D} \frac{\partial \mathcal{L}}{\partial G_{B C}}=0,
$$

where $\Gamma_{A B}^{C}$ is the Christoffel symbol of a local chart in $\mathcal{B}_{t}$.

Proof: The derivative of the action with respect to horizontal variations is computed as follows:

$$
\begin{aligned}
\mathbf{d} S(\Xi, \phi) \cdot(\delta \Xi, 0)= & \int_{t_{0}}^{t_{1}} \int_{\mathcal{B}}\left(\left[\frac{\partial \mathcal{L}}{\partial \Xi} \cdot \delta \Xi-\frac{\partial \mathcal{L}}{\partial \dot{\varphi} \circ \Xi} \cdot \delta\left(\mathbf{F}_{\phi} \mathbf{F}_{\Xi}^{-1} \circ \Xi \cdot \dot{\Xi}\right)+\frac{\partial \mathcal{L}}{\partial \mathbf{F} \circ \Xi}: \delta\left(\mathbf{F}_{\phi} \mathbf{F}_{\Xi}^{-1} \circ \Xi\right)\right] J_{\Xi}\right. \\
& \left.+\frac{\partial \mathcal{L}}{\partial \mathbf{G} \circ \Xi}: \delta \mathbf{G} \circ \Xi+\mathcal{L} \delta J_{\Xi}\right) \mathrm{d} V(U) \mathrm{d} t=0 .
\end{aligned}
$$

Note that

$$
\delta\left(\mathbf{F}_{\phi} \mathbf{F}_{\Xi}^{-1} \circ \Xi\right)=\mathbf{F}_{\phi} \delta\left(\mathbf{F}_{\Xi}^{-1} \circ \Xi\right)
$$

But

$$
\delta\left(\mathbf{F}_{\Xi}^{-1} \circ \Xi\right)=-\mathbf{F}_{\Xi}^{-1} D(\delta \Xi) \mathbf{F}_{\Xi}^{-1} \circ \Xi
$$

Thus

$$
\delta\left(\mathbf{F}_{\phi} \mathbf{F}_{\Xi}^{-1} \circ \Xi\right)=-\mathbf{F}_{\phi} \mathbf{F}_{\Xi}^{-1} D(\delta \Xi) \mathbf{F}_{\Xi}^{-1} \circ \Xi=-\mathbf{F} D(\delta \Xi) \mathbf{F}_{\Xi}^{-1} \circ \Xi .
$$

Similarly 


$$
\delta\left(\mathbf{F}_{\phi} \mathbf{F}_{\Xi}^{-1} \circ \Xi \cdot \dot{\Xi}\right)=-\mathbf{F} D(\delta \Xi) \mathbf{F}_{\Xi}^{-1} \circ \Xi \cdot \mathbf{W}+\mathbf{F} \circ \Xi \cdot \frac{d}{\mathrm{~d} t}(\delta \Xi) .
$$

In coordinates,

$$
D(\delta \Xi)=\frac{\partial \delta \Xi^{A}}{\partial U^{\beta}} \mathbf{e}_{A} \otimes \mathbf{E}^{\beta}
$$

The second term in (3.52) has two parts which are simplified as follows. The first part is

$$
\begin{gathered}
\int_{t_{0}}^{t_{1}} \int_{\mathcal{B}}\left(\frac{\partial \mathcal{L}}{\partial \dot{\varphi} \circ \Xi}\right)_{a} F_{A}^{a} \circ \Xi \frac{\partial \delta \Xi^{A}}{\partial U^{\beta}}\left(\mathbf{F}_{\Xi}^{-1} \circ \Xi\right)^{\beta}{ }_{B} W^{B} J_{\Xi} \mathrm{d} V(U) \mathrm{d} t=-\int_{t_{0}}^{t_{1}} \int_{\mathcal{B}^{\prime}} \frac{\partial}{\partial X^{B}}\left[\left(\frac{\partial \mathcal{L}}{\partial \dot{\varphi}}\right)_{a} F_{A}^{a}\right] W^{B} \delta \Xi^{A} \\
\circ \Xi^{-1} \mathrm{~d} V(X) \mathrm{d} t-\int_{t_{0}}^{t_{1}} \int_{\mathcal{B}^{\prime}}\left(\frac{\partial \mathcal{L}}{\partial \dot{\varphi}}\right)_{a} F_{A}^{a} W^{B}{ }_{\mid B} \delta \Xi^{A} \circ \Xi^{-1} \mathrm{~d} V(X) \mathrm{d} t
\end{gathered}
$$

Similarly, the second part is simplified as

$$
\begin{aligned}
-\int_{t_{0}}^{t_{1}} \int_{\mathcal{B}} \frac{\partial \mathcal{L}}{\partial \dot{\varphi} \circ \Xi} \cdot \mathbf{F} \circ \Xi & \cdot \frac{d}{\mathrm{~d} t}(\delta \Xi) J_{\Xi} \mathrm{d} V(U) \mathrm{d} t=\int_{t_{0}}^{t_{1}} \int_{\mathcal{B}_{t}} \frac{d}{\mathrm{~d} t}\left[\left(\frac{\partial \mathcal{L}}{\partial \dot{\varphi}}\right)_{a} F_{A}^{a}\right] \delta \Xi^{A} \circ \Xi^{-1} \mathrm{~d} V(X) \mathrm{d} t \\
& +\int_{t_{0}}^{t_{1}} \int_{\mathcal{B}_{t}}\left(\frac{\partial \mathcal{L}}{\partial \dot{\varphi}}\right)_{a} F_{A}^{a} W^{B}{ }_{B} \delta \Xi^{A} \mathrm{~d} V(X) \mathrm{d} t
\end{aligned}
$$

Adding (3.58) and (3.59), the second term of (3.52) can be written as

$$
-\int_{t_{0}}^{t_{1}} \int_{\mathcal{B}} \frac{\partial \mathcal{L}}{\partial \dot{\varphi} \circ \Xi} \delta\left(\mathbf{F}_{\phi} \mathbf{F}_{\Xi}^{-1} \circ \Xi \cdot \dot{\Xi}\right) J_{\Xi} \mathrm{d} V(U) \mathrm{d} t=\int_{t_{0}}^{t_{1}} \int_{\mathcal{B}_{t}} \frac{d}{\mathrm{~d} t}\left[\left(\frac{\partial \mathcal{L}}{\partial \dot{\varphi}}\right)_{a} F_{A}^{a}\right] \delta \Xi^{A} \circ \Xi^{-1} \mathrm{~d} V(X) \mathrm{d} t .
$$

After some lengthy manipulations, the third term of (3.52) is simplified to

$$
\begin{aligned}
\int_{t_{0}}^{t_{1}} \int_{\mathcal{B}} \frac{\partial \mathcal{L}}{\partial \mathbf{F} \circ \Xi}: \delta\left(\mathbf{F}_{\phi} \mathbf{F}_{\Xi}^{-1} \circ \Xi\right) J_{\Xi} \mathrm{d} V(U) \mathrm{d} t \\
=\int_{t_{0}}^{t_{1}} \int_{\mathcal{B}_{t}}\left[\left(\frac{\partial \mathcal{L}}{\partial \mathbf{F}}\right)_{a}^{B} F^{a}{ }_{A}\right]_{\mid B} \delta \Xi^{A} \circ \Xi^{-1} \mathrm{~d} V(X) \mathrm{d} t \\
\quad+\int_{t_{0}}^{t_{1}} \int_{\mathcal{B}_{t}}\left(\frac{\partial \mathcal{L}}{\partial \mathbf{F}}\right)_{a}^{B} F^{a}{ }_{C} \Gamma_{A B}^{C} \delta \Xi^{A} \circ \Xi^{-1} \mathrm{~d} V(X) \mathrm{d} t .
\end{aligned}
$$

The fourth term of (3.52) is simplified to

$$
\int_{t_{0}}^{t_{1}} \int_{\mathcal{B}} \frac{\partial \mathcal{L}}{\partial \mathbf{G} \circ \Xi}: \delta \mathbf{G} \circ \Xi J_{\Xi} \mathrm{d} V(U) \mathrm{d} t=\int_{t_{0}}^{t_{1}} \int_{\mathcal{B}_{t}} 2 G_{C D} \Gamma_{A B}^{D} \frac{\partial \mathcal{L}}{\partial G_{B C}} \mathrm{~d} V(X) \mathrm{d} t
$$

Note that

$$
J_{\Xi}=\left(\operatorname{det} \mathbf{F}_{\Xi}\right) \sqrt{\frac{\operatorname{det} \mathbf{G}}{\operatorname{det} \mathbf{G}_{0}}}
$$

where $\mathbf{G}_{0}$ is the fixed Riemannian metric of the fixed reference configuration. Thus, 


$$
\delta J_{\Xi}=\delta\left(\operatorname{det} \mathbf{F}_{\Xi}\right) \sqrt{\frac{\operatorname{det} \mathbf{G}}{\operatorname{det} \mathbf{G}_{0}}}+\left(\operatorname{det} \mathbf{F}_{\Xi}\right) \frac{\delta \operatorname{det} \mathbf{G}}{\operatorname{det} \mathbf{G}_{0}}=J_{\Xi}\left(\mathbf{F}_{\Xi}^{-1}\right)^{\beta}{ }_{B} \frac{\partial \delta \Xi^{B}}{\partial U^{\beta}}+\left(\operatorname{det} \mathbf{F}_{\Xi}\right) \frac{1}{\sqrt{\operatorname{det} \mathbf{G}_{0}}} \frac{\partial \sqrt{\operatorname{det} \mathbf{G}}}{\partial \mathbf{X}} \delta \Xi
$$

Note that

$$
\frac{\partial \sqrt{\operatorname{det} \mathbf{G}}}{\partial \mathbf{X}}=\frac{1}{2} \sqrt{\operatorname{det} \mathbf{G}} \mathbf{G}^{-1} \frac{\partial \mathbf{G}}{\partial \mathbf{X}}=\sqrt{\operatorname{det} \mathbf{G}} \Gamma_{A B}^{B} \delta \Xi^{A} .
$$

Hence

$$
\delta J_{\Xi}=J_{\Xi}\left(\mathbf{F}_{\Xi}^{-1}\right)^{\beta}{ }_{B} \frac{\partial \delta \Xi^{B}}{\partial U^{\beta}}+J_{\Xi} \Gamma_{A B}^{B} .
$$

Thus the last term of (3.52) is simplified to

$$
\int_{t_{0}}^{t_{1}} \int_{\mathcal{B}} \mathcal{L} \delta J_{\Xi} \mathrm{d} V(U) \mathrm{d} t=-\int_{t_{0}}^{t_{1}} \int_{\mathcal{B}_{t}}\left(\mathcal{L} \delta_{A}^{B}\right)_{\mid B} \delta \Xi^{A} \circ \Xi^{-1} \mathrm{~d} V(X) \mathrm{d} t .
$$

Now substituting the above five simplified terms into (3.52), we have

$$
\begin{aligned}
\mathbf{d} S(\Xi, \phi) \cdot(\delta \Xi, 0) & =\int_{t_{0}}^{t_{1}} \int_{\mathcal{B}_{t}}\left\{\frac{\partial \mathcal{L}}{\partial X^{A}}+\frac{\partial}{\partial t}\left[\left(\frac{\partial \mathcal{L}}{\partial \dot{\varphi}}\right)_{a} F^{a}{ }_{A}\right]-\left[\mathcal{L} \delta_{A}{ }^{B}-\left(\frac{\partial \mathcal{L}}{\partial \mathbf{F}}\right)_{a}^{B} F^{a}{ }_{A}\right]_{\mid B}\right\} \delta \Xi^{A} \\
& \circ \Xi^{-1} \mathrm{~d} V(X) \mathrm{d} t+\int_{t_{0}}^{t_{1}} \int_{\mathcal{B}_{t}}\left\{\left(\frac{\partial \mathcal{L}}{\partial \mathbf{F}}\right)_{a}{ }_{a} F_{C}{ }_{C} \Gamma_{A B}^{C}+2 G_{C D} \Gamma_{A B}^{D} \frac{\partial \mathcal{L}}{\partial G_{B C}}\right\} \delta \Xi^{A} \\
& \circ \Xi^{-1} \mathrm{~d} V(X) \mathrm{d} t=0 .
\end{aligned}
$$

Because $\delta \Xi^{A}$ is arbitrary, we conclude that

$$
\frac{\partial \mathcal{L}}{\partial X^{A}}+\frac{\partial}{\partial t}\left[\left(\frac{\partial \mathcal{L}}{\partial \dot{\varphi}}\right)_{a} F_{A}^{a}\right]-\left[\mathcal{L} \delta_{A}{ }^{B}-\left(\frac{\partial \mathcal{L}}{\partial \mathbf{F}}\right)_{a}^{B} F_{A}^{a}\right]_{\mid B}+\left(\frac{\partial \mathcal{L}}{\partial \mathbf{F}}\right)_{a}^{B} F_{C}^{a} \Gamma_{A B}^{C}+2 G_{C D} \Gamma_{A B}^{D} \frac{\partial \mathcal{L}}{\partial G_{B C}}=0 .
$$

We now show that this is equivalent to the classical Euler-Lagrange equations and does not give us any new information. After some lengthy manipulations, it can be shown that

$$
\begin{aligned}
\frac{\partial \mathcal{L}}{\partial X^{A}} & +\frac{\partial}{\partial t}\left[\left(\frac{\partial \mathcal{L}}{\partial \dot{\varphi}}\right)_{a} F_{A}^{a}\right]-\left[\mathcal{L} \delta_{A}{ }^{B}-\left(\frac{\partial \mathcal{L}}{\partial \mathbf{F}}\right)_{a}^{B} F_{A}^{a}\right]_{\mid B}=\left[\frac{\partial \mathcal{L}}{\partial \varphi^{a}}-\frac{\partial}{\partial t}\left(\frac{\partial \mathcal{L}}{\partial \dot{\varphi}}\right)_{a}-\left(\frac{\partial \mathcal{L}}{\partial \mathbf{F}}\right)_{a \mid A}^{A}\right. \\
& \left.-\left(\frac{\partial \mathcal{L}}{\partial \mathbf{F}}\right)_{b}^{A} F_{A}^{c} \gamma_{a c}^{b}+2 \frac{\partial \mathcal{L}}{\partial g_{c d}} g_{b d} \gamma_{a c}^{b}\right] F_{A}^{a}-\left(\frac{\partial \mathcal{L}}{\partial \mathbf{F}}\right)_{a}^{B} F_{C}^{a} \Gamma_{A B}^{C}-2 G_{C D} \Gamma_{A B}^{D} \frac{\partial \mathcal{L}}{\partial G_{B C}} .
\end{aligned}
$$

[It will be seen in Sec. VI that material covariance of internal energy density implies that the sum of the last two terms is zero. In Sec. VII, it will be shown that material covariance of Lagrangian density results in the same identity. However, at this point there is no such relation and the variational principle does not give us any new information.] This result is known for the case where the underlying metrics are trivial. ${ }^{25}$ In conclusion, we have proved the following proposition.

Proposition 3.6: In the absence of discontinuities, i.e., when all the fields are smooth, the configurational and the standard equations of motion are equivalent, even if one is allowed to vary the referential and spatial metrics. 


\section{THE GREEN-NAGHDI-RIVILIN THEOREM}

Green, Rivilin, and Naghdi ${ }^{19}$ realized that conservation of mass and balance of linear and angular momenta can be obtained as a result of postulating invariance of energy balance under isometries of $\mathbb{R}^{3}$, i.e., rigid translations and rotations in the deformed configuration. Later Marsden and Hughes ${ }^{28}$ extended this idea to Riemannian manifolds and diffeomorphisms of the deformed configuration showing that this covariant approach gives the Doyle-Ericksen formula for Cauchy stress as well as conservation of mass and balance of linear and angular momenta. In another relevant work, Šilhavý ${ }^{39}$ considered all the densities in the energy balance to be volume densities and assuming (i) invariance of energy balance under Galilean transformations and (ii) boundedness of energy from below, proved the existence of mass, its conservation, balance of linear and angular momenta, transformation of body forces and the splitting of total energy into internal and kinetic energies.

Before discussing the covariant approach to elasticity, let us first discuss the classical GreenNaghdi-Rivilin (GNR) theorem and a nonconventional material form of it. We consider two cases: (i) material energy balance invariance under spatial isometries of $\mathbb{R}^{3}$ and (ii) material energy balance invariance under material isometries of $\mathbb{R}^{3}$. We call (i) and (ii) the spatial-material and material-material GNR theorems, respectively.

\section{A. The spatial-material GNR theorem}

Consider the material energy balance for a nice subset $\mathcal{U} \subset \mathcal{B}$,

$$
\frac{d}{\mathrm{~d} t} \int_{\mathcal{U}} \rho_{0}\left(\Psi+\frac{1}{2} \mathbf{V} \cdot \mathbf{V}\right) \mathrm{d} V=\int_{\mathcal{U}} \rho_{0}(\mathbf{B} \cdot \mathbf{V}+R) \mathrm{d} V+\int_{\partial \mathcal{U}}(\mathbf{T} \cdot \mathbf{V}+H) \mathrm{d} A,
$$

where $\Psi=\Psi(t, \mathbf{X}, \mathbf{F})$ is the free energy density per unit mass of the undeformed configuration. Now consider an isometry $\xi_{t}: \mathbb{R}^{3} \rightarrow \mathbb{R}^{3}$ of $\mathbb{R}^{3}$. We postulate that the material energy balance is invariant under $\xi_{t}$. For the sake of simplicity we consider translations and rotations separately.

(i) (Rigid translations) A spatial rigid translation is defined by

$$
\xi_{t}(\mathbf{x})=\mathbf{x}+\left(t-t_{0}\right) \mathbf{c},
$$

where $\mathbf{c}$ is some constant vector field. We now postulate that the material balance of energy holds for the deformation mapping $\varphi_{t}^{\prime}=\xi_{t} \varphi_{t}$ as well. This balance law is still written on $\mathcal{U}$ but with different fields (primed fields) in general,

$$
\frac{d}{\mathrm{~d} t} \int_{\mathcal{U}} \rho_{0}^{\prime}\left(\Psi^{\prime}+\frac{1}{2} \mathbf{V}^{\prime} \cdot \mathbf{V}^{\prime}\right) \mathrm{d} V=\int_{\mathcal{U}} \rho_{0}^{\prime}\left(\mathbf{B}^{\prime} \cdot \mathbf{V}^{\prime}+R^{\prime}\right) \mathrm{d} V+\int_{\partial \mathcal{U}}\left(\mathbf{T}^{\prime} \cdot \mathbf{V}^{\prime}+H^{\prime}\right) \mathrm{d} A
$$

Using Cartan's space-time theory, the primed fields are related to the unprimed quantities through the following relations:

$$
\begin{gathered}
\rho_{0}^{\prime}(\mathbf{X})=\rho_{0}(\mathbf{X}), \quad R^{\prime}(\mathbf{X})=R(\mathbf{X}), \quad H^{\prime}(\mathbf{X})=H(\mathbf{X}), \\
\left.\mathbf{V}^{\prime}\right|_{t=t_{0}}=\left.\frac{\partial}{\partial t} \varphi_{t}^{\prime}\right|_{t=t_{0}}=\left(T \xi_{t} \mathbf{V}+\mathbf{c}\right)_{t=t_{0}}=\mathbf{V}+\mathbf{c}, \\
\mathbf{T}^{\prime}(\mathbf{X}, \mathbf{N})=\mathbf{T}(\mathbf{X}, \mathbf{N}) .
\end{gathered}
$$

Also because 


$$
\mathbf{b}^{\prime}-\mathbf{a}^{\prime}=\xi_{t^{*}}(\mathbf{b}-\mathbf{a}) \quad \text { and } \quad \mathbf{B}-\mathbf{A}=(\mathbf{b}-\mathbf{a}) \circ \varphi_{t}
$$

We have

$$
\mathbf{B}^{\prime}-\mathbf{A}^{\prime}=\xi_{t}^{*}(\mathbf{b}-\mathbf{a}) \circ \varphi_{t}^{\prime}
$$

Hence

$$
\left(\mathbf{B}^{\prime}-\mathbf{A}^{\prime}\right)_{t=t_{0}}=(\mathbf{b}-\mathbf{a}) \circ \varphi_{t}=(\mathbf{B}-\mathbf{A}) .
$$

It can be easily shown that

$$
\mathbf{F}^{\prime}(\mathbf{X})=\mathbf{F}(\mathbf{X}) \text {. }
$$

The free energy density would have the following transformation:

$$
\Psi^{\prime}\left(t, \mathbf{X}, \mathbf{F}^{\prime}(\mathbf{X})\right)=\Psi(t, \mathbf{X}, \mathbf{F}(\mathbf{X}))
$$

Thus,

$$
\frac{d}{\mathrm{~d} t} \Psi^{\prime}\left(t, \mathbf{X}, \mathbf{F}^{\prime}(\mathbf{X})\right)=\frac{\partial \Psi}{\partial t} .
$$

Balance of energy for $\mathcal{U} \subset \mathcal{B}$ for the new deformation mapping at $t=t_{0}$ can be written as

$$
\begin{aligned}
\int_{\mathcal{U}} \frac{\partial \rho_{0}}{\partial t}\left(\Psi+\frac{1}{2}(\mathbf{V}+\mathbf{c}) \cdot(\mathbf{V}+\mathbf{c})\right) \mathrm{d} V+\int_{\mathcal{U}} \rho_{0}\left(\frac{\partial \Psi}{\partial t}+\left.(\mathbf{V}+\mathbf{c}) \cdot \mathbf{A}^{\prime}\right|_{t=t_{0}}\right) \mathrm{d} V \\
\quad=\int_{\mathcal{U}} \rho_{0}\left(\left.\mathbf{B}^{\prime}\right|_{t=t_{0}} \cdot(\mathbf{V}+\mathbf{c})+R\right) \mathrm{d} V+\int_{\partial \mathcal{U}}(\mathbf{T} \cdot(\mathbf{V}+\mathbf{c})+H) \mathrm{d} A
\end{aligned}
$$

where Div $\mathbf{c}=0$ was used. Subtracting the material energy balance of the deformation $\varphi_{t}$ for $\mathcal{U} \subset \mathcal{B}$ from the above equation and using (4.7) we obtain

$$
\int_{\mathcal{U}} \frac{\partial \rho_{0}}{\partial t}\left(\mathbf{c} \cdot \mathbf{V}+\frac{1}{2} \mathbf{c} \cdot \mathbf{c}\right) \mathrm{d} V+\int_{\mathcal{U}} \rho_{0} \mathbf{A} \cdot \mathbf{c} \mathrm{d} V=\int_{\mathcal{U}} \rho_{0} \mathbf{B} \cdot \mathbf{c} \mathrm{d} V+\int_{\partial \mathcal{U}} \mathbf{T} \cdot \mathbf{c} \mathrm{d} A
$$

Because $\mathcal{U}$ and $\mathbf{c}$ are arbitrary one concludes that

$$
\frac{\partial \rho_{0}}{\partial t}=0
$$

$$
\operatorname{Div} \mathbf{P}+\rho_{0} \mathbf{B}=\rho_{0} \mathbf{A} .
$$

(ii) (Rigid rotations) Now let us consider a rigid rotation in the ambient space, i.e., $\xi_{t}: \mathcal{S}$ $\rightarrow \mathcal{S}$, where

$$
\xi_{t}(\mathbf{x})=e^{\left(t-t_{0}\right) \mathbf{\Omega}} \mathbf{x},
$$

for some constant skew-symmetric matrix $\boldsymbol{\Omega}$. Note that

$$
\left.T \xi_{t}\right|_{t=t_{0}}=\left.e^{\left(t-t_{0}\right) \boldsymbol{\Omega}}\right|_{t=t_{0}}=\mathrm{Id} \quad \text { and }\left.\quad \frac{\partial}{\partial t}\right|_{t=t_{0}} \xi_{t}(\mathbf{x})=\mathbf{\Omega} \mathbf{x}
$$

Also 


$$
\left.\mathbf{V}^{\prime}(\mathbf{X})\right|_{t=t_{0}}=\mathbf{V}+\mathbf{\Omega x}(\mathbf{X}) .
$$

Subtracting the balance of energy for $\mathcal{U}$ for deformation mapping $\varphi_{t}$ from that of $\varphi_{t}^{\prime}$ $=\xi_{t} \circ \varphi_{t}$ at time $t=t_{0}$ results in

$$
\int_{\mathcal{U}} \rho_{0} \boldsymbol{\Omega} \mathbf{x}(\mathbf{X}) \cdot(\mathbf{A}-\mathbf{B}) \mathrm{d} V=\int_{\partial \mathcal{U}} \mathbf{T} \boldsymbol{\Omega} \mathbf{x}(\mathbf{X}) \mathrm{d} A .
$$

But

$$
\int_{\partial \mathcal{U}} \mathbf{T} \boldsymbol{\Omega} \mathbf{x}(\mathbf{X}) \mathrm{d} A=\int_{\mathcal{U}}\left(\operatorname{Div} \mathbf{P} \cdot \mathbf{\Omega} \mathbf{x}+\mathbf{P F}^{\top}: \mathbf{\Omega}\right) \mathrm{d} V .
$$

Thus

$$
\mathbf{P F}^{\top}=\mathbf{F P}^{\top},
$$

where use was made of balance of linear momentum.

\section{B. The material-material GNR theorem}

To our best knowledge, there is no study of invariance of energy balance under isometries of the reference configuration in the literature. It turns out that such an invariance does not hold in general, even in Euclidean space. In this section we study the transformation of balance of energy under rigid translations and rotations of the reference configuration in the Euclidean space context. It will be shown that balance of energy is invariant under translations and rotations of the reference configuration for isotropic materials that satisfy an internal constraint that we call material inviscidity.

Again we consider rigid translations and rigid rotations of the reference configuration separately.

(i) (Rigid translations) Consider a time-dependent rigid translation of the reference configuration $\Xi_{t}: \mathcal{B} \rightarrow \mathcal{B}^{\prime}$. Let

$$
\mathbf{X}^{\prime}=\mathbf{X}_{t}=\Xi_{t}(\mathbf{X})=\mathbf{X}+\left(t-t_{0}\right) \mathbf{W},
$$

for some constant vector field $\mathbf{W}$. Note that

$$
T \Xi_{t}=\mathrm{Id}, \quad \mathbf{X}=\Xi_{t}^{-1}\left(\mathbf{X}_{t}\right)=\mathbf{X}_{t}-\left(t-t_{0}\right) \mathbf{W} .
$$

Deformation gradient with respect to the new reference configuration is denoted $\mathbf{F}^{\prime}$ and,

$$
\mathrm{d} \mathbf{x}=\mathbf{F} \mathrm{d} \mathbf{X}=\mathbf{F}^{\prime} \mathrm{d} \mathbf{X}^{\prime} .
$$

But, $\mathrm{d} \mathbf{X}^{\prime}=\mathrm{d} \mathbf{X}$ and hence

$$
\mathbf{F} \mathrm{d} \mathbf{X}=\mathbf{F}^{\prime} \mathrm{d} \mathbf{X} \quad \forall \mathrm{d} \mathbf{X} .
$$

This means that

$$
\mathbf{F}^{\prime}\left(\mathbf{X}_{t}\right)=\mathbf{F}(\mathbf{X}) \quad \text { or } \quad \mathbf{F}^{\prime}=\mathbf{F} \circ \Xi_{t}^{-1} .
$$

In the differential geometry language this means that

$$
\mathbf{F}^{\prime}=\Xi_{t^{*}} \mathbf{F}=\mathbf{F} \circ \Xi_{t}^{-1}
$$

The material velocity with respect to the new reference configuration is 


$$
\mathbf{V}^{\prime}\left(\mathbf{X}_{t}\right)=\frac{\partial}{\partial t} \varphi_{t}^{\circ} \Xi_{t}^{-1}\left(\mathbf{X}^{\prime}\right)=\mathbf{V} \circ \Xi_{t}^{-1}\left(\mathbf{X}^{\prime}\right)-\mathbf{F W}
$$

Thus at $t=t_{0}$,

$$
\mathbf{V}^{\prime}=\mathbf{V}-\mathbf{F W} .
$$

Free energy density is assumed to have the following transformation:

$$
\Psi^{\prime}\left(\mathbf{X}^{\prime}, \mathbf{F} \circ \Xi_{t}^{-1}\right)=\Psi(\mathbf{X}, \mathbf{F})
$$

Or

$$
\Psi^{\prime}\left(\mathbf{X}^{\prime}, \mathbf{F}\right)=\Psi\left(\mathbf{X}, \mathbf{F} \circ \Xi_{t}\right) .
$$

[Note that this does not put any restrictions on the material properties as here all we assume is that under a change of frame the 3-form $\rho_{0} \Psi \mathrm{d} V$ is transformed to a 3-form $\rho_{0}^{\prime} \Psi^{\prime} \mathrm{d} V^{\prime}=\Xi_{t^{*}}\left(\rho_{0} \Psi \mathrm{d} V\right)$.] More precisely,

$$
\Xi_{t}^{*} \Psi^{\prime}\left(\mathbf{X}^{\prime}, \mathbf{F}\right)=\Psi\left(\mathbf{X}, \mathbf{F} \circ \Xi_{t}\right) .
$$

Thus

$$
\frac{d}{\mathrm{~d} t} \Psi^{\prime}\left(\mathbf{X}^{\prime}, \mathbf{F}\right)=\frac{\partial \Psi}{\partial t}+\frac{\partial \Psi}{\partial\left(\mathbf{F} \circ \Xi_{t}\right)}: \frac{\partial \mathbf{F}}{\partial \Xi_{t}(\mathbf{X})} . \mathbf{W} .
$$

Hence at $t=t_{0}$

$$
\frac{d}{\mathrm{~d} t} \Psi^{\prime}\left(\mathbf{X}^{\prime}, \mathbf{F}\right)=\frac{\partial \Psi}{\partial t}+\frac{\partial \Psi}{\partial \mathbf{F}}: \frac{\partial \mathbf{F}}{\partial \mathbf{X}} . \mathbf{W} .
$$

Material balance of energy for $\mathcal{U} \subset \mathcal{B}$ reads

$$
\begin{gathered}
\int_{\mathcal{U}} \frac{\partial \rho_{0}}{\partial t}\left(\Psi+\frac{1}{2}\langle\langle\mathbf{V}, \mathbf{V}\rangle\rangle\right) \mathrm{d} V+\int_{\mathcal{U}} \rho_{0}\left(\frac{d}{\mathrm{~d} t} \Psi+\langle\langle\mathbf{V}, \mathbf{A}\rangle\rangle\right) \mathrm{d} V \\
=\int_{\mathcal{U}} \rho_{0}(\mathbf{B} \cdot \mathbf{V}+R) \mathrm{d} V+\int_{\partial \mathcal{U}}(\mathbf{T} \cdot \mathbf{V}+H) \mathrm{d} A .
\end{gathered}
$$

Let us assume that material balance of energy for $\mathcal{U}^{\prime} \subset \mathcal{B}^{\prime}$ reads

$$
\begin{aligned}
\frac{d}{\mathrm{~d} t} \int_{\mathcal{U}^{\prime}} \rho_{0}^{\prime}\left(\Psi^{\prime}+\frac{1}{2} \mathbf{V}^{\prime} \cdot \mathbf{V}^{\prime}\right) \mathrm{d} V^{\prime}= & \int_{\mathcal{U}^{\prime}} \rho_{0}\left(\mathbf{B}^{\prime} \cdot \mathbf{V}^{\prime}+R^{\prime}\right) \mathrm{d} V^{\prime}+\int_{\mathcal{U}^{\prime}} \mathbf{B}_{0}^{\prime} \cdot \mathbf{W}_{t} \mathrm{~d} V^{\prime} \\
& +\int_{\partial \mathcal{U}^{\prime}}\left(\mathbf{T}^{\prime} \cdot \mathbf{V}^{\prime}+H^{\prime}\right) \mathrm{d} A^{\prime},
\end{aligned}
$$

for some vector field $\mathbf{B}_{0}^{\prime}$ which will be determined shortly. Note that thinking of the integrand of the left-hand side of balance of energy as a 3-form $\alpha$, we have

$$
\frac{d}{\mathrm{~d} t} \int_{\mathcal{U}^{\prime}} \alpha^{\prime}=\int_{\mathcal{U}} \frac{d}{\mathrm{~d} t}\left(\Xi_{t}^{*} \alpha^{\prime}\right)
$$

But $\Xi_{t}^{*} \alpha^{\prime}=\rho_{0}(\mathbf{X}) \Psi\left(\mathbf{X}, \mathbf{F} \circ \Xi_{t}\right) \mathrm{dV}$, thus material balance of energy for $\mathcal{U}^{\prime} \subset \mathcal{B}^{\prime}$ at $t=t_{0}$ reads 


$$
\begin{gathered}
\int_{\mathcal{U}} \frac{\partial \rho_{0}}{\partial t}\left(\Psi+\frac{1}{2}\langle\langle\mathbf{V}-\mathbf{F W}, \mathbf{V}-\mathbf{F W}\rangle\rangle\right) \mathrm{d} V+\int_{\mathcal{U}} \rho_{0}\left(\left.\frac{d}{\mathrm{~d} t}\right|_{t=t_{0}} \Psi^{\prime}+\left\langle\left\langle\mathbf{V}-\mathbf{F W},\left.\mathbf{A}^{\prime}\right|_{t=t_{0}}\right\rangle\right\rangle\right) \mathrm{d} V \\
=\int_{\mathcal{U}} \rho_{0}\left(\left.\mathbf{B}^{\prime}\right|_{t=t_{0}} \cdot(\mathbf{V}-\mathbf{F W})+R\right) \mathrm{d} V+\int_{\partial \mathcal{U}}(\mathbf{T} \cdot(\mathbf{V}-\mathbf{F W})+H) \mathrm{d} A+\int_{\mathcal{U}} \mathbf{B}_{0} \cdot \mathbf{W} \mathrm{d} V
\end{gathered}
$$

where $\mathbf{B}_{0}$ is an unknown vector field at this point. Note that

$$
\left(\mathbf{B}^{\prime}-\mathbf{A}^{\prime}\right)_{t=t_{0}}=\mathbf{B}-\mathbf{A} .
$$

Now subtracting the material balance of energy for $\mathcal{U} \subset \mathcal{B}$ from that of $\mathcal{U}^{\prime} \subset \mathcal{B}^{\prime}$ at time $t=t_{0}$ yields

$$
\int_{\mathcal{U}}\left(\mathbf{P}: \frac{\partial \mathbf{F}}{\partial \mathbf{X}}+\rho_{0} \mathbf{F}^{\top}(\mathbf{B}-\mathbf{A})-\mathbf{B}_{0}\right) \cdot \mathbf{W} \mathrm{d} V+\int_{\partial \mathcal{U}} \mathbf{F}^{\top} \mathbf{T} \cdot \mathbf{W} \mathrm{d} A=0 \quad \forall \mathbf{W} .
$$

Localization leads to the following conclusion:

$$
\mathbf{B}_{0}=\operatorname{Div}\left(\mathbf{F}^{\top} \mathbf{P}\right)+\rho_{0} \mathbf{F}^{\top}(\mathbf{B}-\mathbf{A})+\mathbf{P}: \frac{\partial \mathbf{F}}{\partial \mathbf{X}} .
$$

Note that

$$
\mathbf{P}: \frac{\partial \mathbf{F}}{\partial \mathbf{X}}=\operatorname{Div}(\Psi \mathbf{I})-\frac{\partial \Psi}{\partial \mathbf{X}}
$$

and

$$
\operatorname{Div}\left(\mathbf{F}^{\top} \mathbf{P}\right)=\mathbf{F}^{\top} \operatorname{Div} \mathbf{P}+\mathbf{P}: \frac{\partial \mathbf{F}}{\partial \mathbf{X}} .
$$

Thus (4.40) is equivalent to

$$
\mathbf{B}_{0}=\mathbf{F}^{\top}\left[\operatorname{Div} \mathbf{P}+\rho_{0}(\mathbf{B}-\mathbf{A})\right]+2 \mathbf{P}: \frac{\partial \mathbf{F}}{\partial \mathbf{X}}=2 \mathbf{P}: \frac{\partial \mathbf{F}}{\partial \mathbf{X}} .
$$

Therefore, the transformed balance of energy is (4.35) with $\mathbf{B}_{0}^{\prime}=\Xi_{t^{*}}\left(\mathbf{B}_{0}\right)$.

Invariance of balance of energy under rigid translations of the reference configuration is equivalent to $\mathbf{B}_{0}=\mathbf{0}$, i.e.,

$$
\mathbf{P}: \frac{\partial \mathbf{F}}{\partial \mathbf{X}}=\mathbf{0}
$$

which is equivalent to

$$
\operatorname{Div}\left(\mathbf{F}^{\top} \mathbf{P}\right)=\mathbf{F}^{\top} \operatorname{Div}(\mathbf{P}) .
$$

Obviously, if $\mathbf{F}$ is independent of $\mathbf{X}$, i.e., if the deformation gradient is uniform then this condition is satisfied but as we will see in the sequel this is not necessary. Note that (4.43) is independent of balance of linear momentum. It is seen that an additional constraint must be satisfied for the material energy balance to be invariant under time-dependent rigid referential translations. This shows the very different natures of material and spatial manifolds. We will show at the end of Sec. VI that (4.45) implies that configurational stress tensor is hydrostatic. For this reason we call (4.45) the configurational inviscidity constraint.

Example: Consider a Neo-Hookean rod in uniaxial tension. The deformation gradient is 


$$
\mathbf{F}=\left(\begin{array}{lll}
\lambda^{-1 / 2} & 0 & 0 \\
0 & \lambda^{-1 / 2} & 0 \\
0 & 0 & \lambda
\end{array}\right) .
$$

It can be easily shown that the first Piola-Kirchhoff stress tensor has the following representation:

$$
\mathbf{P}=\left(\begin{array}{ccc}
0 & 0 & 0 \\
0 & 0 & 0 \\
0 & 0 & \mu \lambda-\frac{\mu}{\lambda^{2}}
\end{array}\right),
$$

where $\mu=\mu(\mathbf{X})$. It is now an easy exercise to show that (4.45) is satisfied only if $\lambda$ is constant, i.e., only if the deformation gradient is uniform. Thus in this case the only possibility would be a uniform deformation gradient for balance of energy to be invariant under rigid translations of the reference configuration.

Example: We know that for an isotropic material

$$
S_{A B}=\alpha_{0} G_{A B}+\alpha_{1} C_{A B}+\alpha_{2} C_{A}^{D} C_{D B},
$$

where $\alpha_{0}, \alpha_{1}$, and $\alpha_{2}$ are scalar functions of $\mathbf{X}$ and $S_{A B}$ are components of the second Piola-Kirchhoff stress tensor. For the sake of simplicity, suppose $\alpha_{1}=\alpha_{2}=0$. In terms of $\mathbf{P}$ and $\mathbf{F}$ we have

$$
P^{a A}=\alpha_{0} G^{A B} F^{a}{ }_{B} .
$$

When the reference configuration and ambient space are Euclidean the condition $\operatorname{Div}\left(\mathbf{F}^{\top} \mathbf{P}\right)=\mathbf{F}^{\top} \operatorname{Div}(\mathbf{P})$ is equivalent to

$$
\alpha_{0} F_{B}^{a} \frac{\partial F_{B}^{a}}{\partial X^{A}}=0 .
$$

Or

$$
F_{B}^{a} \frac{\partial F_{B}^{a}}{\partial X^{A}}=F_{B}^{a} \frac{\partial F_{A}^{a}}{\partial X^{B}}=0 .
$$

Note that, in general, this does not imply that the deformation gradient is uniform and it is simply an internal constraint.

Example: Consider an incompressible perfect fluid (ideal fluid) for which

$$
\sigma^{a b}=-p g^{a b} \quad \text { and } \quad J=1 .
$$

Thus

$$
P^{a A}=-J\left(\mathbf{F}^{-1}\right)^{A}{ }_{b} p g^{a b} .
$$

Using Piola identity we have

$$
\left(\operatorname{Div}\left(\mathbf{F}^{\top} \mathbf{P}\right)\right)^{A}=\left(-p J G^{A B}\right)_{\mid B}=-J \frac{\partial p}{\partial x^{b}} F_{B}^{b} G^{A B}
$$

Also

$$
\left(\mathbf{F}^{\top} \operatorname{Div}(\mathbf{P})\right)^{A}=-g_{a b} F_{B}^{b} G^{A B} J\left(p g^{a d}\right)_{\mid d}=-J \frac{\partial p}{\partial x^{b}} F_{B}^{b} G^{A B} .
$$

Thus (4.45) is satisfied for an ideal fluid. 
(ii) (Rigid rotations) Consider a time-dependent rigid rotation of the reference configuration $\Xi_{t}: \mathcal{B} \rightarrow \mathcal{B}^{\prime}$ defined as

$$
\mathbf{X}^{\prime}=\mathbf{X}_{t}=e^{\left(t-t_{0}\right) \mathbf{\Omega}} \mathbf{X}
$$

for some constant skew-symmetric matrix $\boldsymbol{\Omega}$. Note that

$$
\mathbf{V}^{\prime}=V-\mathbf{F} \mathbf{\Omega X}, \quad \mathbf{F}^{\prime}=\mathbf{F} \circ \Xi_{t}^{-1} .
$$

Let us assume that material balance of energy for $\mathcal{U}^{\prime} \subset \mathcal{B}^{\prime}$ has the following form:

$$
\begin{aligned}
\frac{d}{\mathrm{~d} t} \int_{\mathcal{U}^{\prime}} \rho_{0}^{\prime}\left(\Psi^{\prime}+\frac{1}{2} \mathbf{V}^{\prime} \cdot \mathbf{V}^{\prime}\right) \mathrm{d} V^{\prime}= & \int_{\mathcal{U}^{\prime}} \rho_{0}\left(\mathbf{B}^{\prime} \cdot \mathbf{V}^{\prime}+R^{\prime}\right) \mathrm{d} V^{\prime}+\int_{\partial \mathcal{U}^{\prime}}\left(\mathbf{T}^{\prime} \cdot \mathbf{V}^{\prime}+H^{\prime}\right) \mathrm{d} A^{\prime} \\
& +\int_{\mathcal{U}^{\prime}}\left(\mathbf{B}_{0}^{\prime} \cdot \mathbf{\Omega} \mathbf{X}+\mathbf{C}_{0}^{\prime}: \mathbf{\Omega}\right) \mathrm{d} V^{\prime}
\end{aligned}
$$

where $\mathbf{C}_{0}^{\prime}=\Xi_{t^{*}} \mathbf{C}_{0}$ and $\mathbf{C}_{0}$ is an unknown vector field at this point. Material balance of energy for $\mathcal{U}^{\prime} \subset \mathcal{B}^{\prime}$ at $t=t_{0}$ reads

$$
\begin{aligned}
\int_{\mathcal{U}} \frac{\partial \rho_{0}}{\partial t}\left(\Psi+\frac{1}{2}\langle\langle\mathbf{V}-\mathbf{F} \mathbf{\Omega} \mathbf{X}, \mathbf{V}-\mathbf{F} \boldsymbol{\Omega} \mathbf{X}\rangle\rangle\right) \mathrm{d} V \\
\quad+\int_{\mathcal{U}} \rho_{0}\left(\left.\frac{d}{\mathrm{~d} t}\right|_{t=t_{0}} \Psi^{\prime}+\left\langle\left\langle\mathbf{V}-\mathbf{F} \mathbf{\Omega} \mathbf{X},\left.\mathbf{A}^{\prime}\right|_{t=t_{0}}\right\rangle\right\rangle\right) \mathrm{d} V \\
=\int_{\mathcal{U}} \rho_{0}\left(\left.\mathbf{B}^{\prime}\right|_{t=t_{0}} \cdot(\mathbf{V}-\mathbf{F} \mathbf{\Omega} \mathbf{X})+R\right) \mathrm{d} V+\int_{\partial \mathcal{U}}(\mathbf{T} \cdot(\mathbf{V}-\mathbf{F} \mathbf{\Omega X})+H) \mathrm{d} A \\
\quad+\int_{\mathcal{U}}\left(\mathbf{B}_{0} \cdot \mathbf{\Omega} \mathbf{X}+\mathbf{C}_{0}: \mathbf{\Omega}\right) \mathrm{d} V .
\end{aligned}
$$

Subtracting the material balance of energy for $\mathcal{U} \subset \mathcal{B}$ from that of $\mathcal{U}^{\prime} \subset \mathcal{B}^{\prime}$ at time $t=t_{0}$ and considering the relation for $\mathbf{B}_{0}$ coming from rigid translations of the reference configuration yields

$$
\int_{\mathcal{U}}\left(\mathbf{F}^{\top} \mathbf{P}-\mathbf{C}_{0}\right): \Omega \mathrm{d} V=0 .
$$

This means that

$$
\mathbf{F}^{\top} \mathbf{P}-\mathbf{C}_{0}=\left(\mathbf{F}^{\top} \mathbf{P}-\mathbf{C}_{0}\right)^{\top} .
$$

Thus $\mathbf{C}_{0}=-\mathbf{P}^{\top} \mathbf{F}+\mathbf{S}$ for some symmetric tensor $\mathbf{S}$. This symmetric tensor does not contribute to balance of energy and we can choose it to be $\mathbf{S}=\mathbf{0}$. Thus the transformed balance of energy under rigid rotations of the reference configuration is (4.58) where $\mathbf{C}_{0}^{\prime}=\Xi_{t^{*}}\left(\mathbf{C}_{0}\right)$ and $\mathbf{C}_{0}=-\mathbf{P}^{\top} \mathbf{F}$.

In conclusion, we have proved the following proposition.

Proposition 4.1: Balance of energy is invariant under time-dependent translations and rotations of the reference configuration if $\mathbf{B}_{0}=\mathbf{C}_{0}=\mathbf{0}$, i.e., if the reference configuration is both configurationally inviscid and isotropic.

Thus, balance of energy is invariant under material isometries of the reference configuration only under some constraints. As an example, it is seen that balance of energy is invariant under material isometries in the case of ideal fluids. 


\section{COVARIANT SPATIAL ENERGY BALANCE}

In this section we start by a reappraisal of the concept of covariance in elasticity and its consequences. We revisit Marsden and Hughes' theorem ${ }^{28}$ and clarify some details in their proof. We then show that the same conclusions can be reached if one assumes that mass density is a 3-form instead of a scalar. A proof is then given for converse of Marsden and Hughes' theorem, i.e., assuming conservation of mass, balance of linear and angular momenta and Doyle-Ericksen formula, balance of energy is invariant under arbitrary spatial diffeomorphisms. At the end of this section, we show that assuming spatial covariance for material energy balance yields results that are identical to those obtained by assuming spatial covariance for spatial energy balance.

\section{A. Covariance and the Doyle-Ericksen formula}

First recall that the general notion of covariance of a set of equations is as follows.

Definition 5.1 (Covariance): Suppose a theory has some tensor fields $\mathbf{U}, \mathbf{V}, \ldots$ defined on a space $\mathcal{A}$ and the governing equations of the theory have the form $F(\mathbf{U}, \mathbf{V}, \ldots)=0$. These governing equations are called covariant if for any diffeomorphism $\xi: \mathcal{A} \rightarrow \mathcal{A}, \xi^{*}(F(\mathbf{U}, \mathbf{V}, \ldots))$ $=F\left(\xi^{*} \mathbf{U}, \xi^{*} \mathbf{V}, \ldots\right)$. A theory is covariant if all its governing equations are covariant.

The Doyle-Ericksen formula: Doyle and Ericksen ${ }^{7}$ showed the following interesting relation:

$$
\boldsymbol{\sigma}=2 \rho \frac{\partial e}{\partial \mathbf{g}},
$$

i.e., Cauchy's stress tensor is proportional to the partial derivative of the free energy density with respect to the Riemannian metric in the deformed configuration. [Note that (see Ref. 28, p. 198)

$$
\frac{\partial e}{\partial \mathbf{g}}=\frac{\partial \psi}{\partial \mathbf{g}} .
$$

In other words, in Doyle-Ericksen formula internal energy density can be replaced by free energy density because

$$
e=\psi+\theta s
$$

where $\theta$ is absolute temperature and $s$ is entropy density. Thus

$$
\frac{\partial e}{\partial \mathbf{g}}=\frac{\partial \psi}{\partial \mathbf{g}}+\frac{\partial \psi}{\partial \theta} \frac{\partial \theta}{\partial \mathbf{g}}+\frac{\partial \theta}{\partial \mathbf{g}} s=\frac{\partial \psi}{\partial \mathbf{g}},
$$

as $\partial \psi / \partial \theta=-s]$.

Doyle and Ericksen ${ }^{7}$ looked at changes of spatial frame passively, i.e., as changes of coordinates while Marsden and Hughes ${ }^{28}$ chose the active point of view. The Doyle-Ericksen formula is known to be the essential condition for covariance of energy balance. Later Simo and Marsden ${ }^{40}$ found a material version of Doyle-Ericksen formula, which we discuss next. Here by "material version" they mean an analogue of the usual Doyle-Ericksen formula that ensures covariance of material energy balance under spatial diffeomorphisms. [An interesting question to ask would be the condition(s) that ensures covariance of material energy balance under diffeomorphisms of the reference configuration. This will be discussed in Sec. VI.] Simo and Marsden consider a general form of polar decomposition theorem by first associating two Riemannian metrics $\mathbf{G}_{0}$ and $\mathbf{G}$ to $\mathcal{B}$, where $\mathbf{G}_{0}$ does not change under spatial diffeomorphisms while $\mathbf{G}$ does change. The polar decomposition theorem states that

$$
\mathbf{F}=\mathbf{R} \mathbf{U},
$$

where 


$$
\mathbf{U}(\mathbf{X}):\left(T_{\mathbf{X}} \mathcal{B}, \mathbf{G}_{0}\right) \rightarrow\left(T_{\mathbf{X}} \mathcal{B}, \mathbf{G}\right)
$$

is the material stretch tensor (a positive-definite symmetric linear map with respect to the given metrics) and

$$
\mathbf{R}(\mathbf{X}):\left(T_{\mathbf{X}} \mathcal{B}, \mathbf{G}\right) \rightarrow\left(T_{\varphi_{t}(\mathbf{X})} \mathcal{S}, \mathbf{g}\right)
$$

is, for each $X \in \mathcal{B}$, a $(\mathbf{G}, \mathbf{g})$-orthogonal linear transformation. The metric $\mathbf{G}$ is arbitrary and can change under spatial diffeomorphisms,

$$
\mathbf{G}=\mathbf{R}^{*}(\mathbf{g}) \text {. }
$$

The internal energy density per unit mass of the deformed configuration is

$$
e=e(\mathbf{x}, t, \mathbf{g}(\mathbf{x})) .
$$

Now define

$$
E(\mathbf{X}, t, \mathbf{G})=e\left(\varphi_{t}(\mathbf{X}), t, \mathbf{R}_{*}(\mathbf{G})\right) .
$$

Simo and Marsden ${ }^{40}$ show that

$$
\Sigma=2 \rho \frac{\partial E}{\partial \mathbf{G}}
$$

where $\mathbf{\Sigma}$ is the rotated stress tensor defined as

$$
\boldsymbol{\Sigma}=\mathbf{R}^{*} \boldsymbol{\sigma} \quad \text { or } \quad \Sigma^{A B}=\left(\mathbf{R}^{-1}\right)_{a}^{A} \sigma^{a b}\left(\mathbf{R}^{-1}\right)^{B}{ }_{b} .
$$

In this paper we prove a similar theorem by postulating a balance of energy for an arbitrary reframing of the reference configuration for a special class of materials. It should be noted that there are four possibilities for a covariant energy balance law.

(i) Spatial energy balance law for any reframing of the deformed configuration: This gives the usual Doyle-Ericksen formula.

(ii) Material energy balance law for any reframing of the deformed configuration: This gives the Doyle-Ericksen formula in terms of Kirchhoff stress tensor.

(iii) Material energy balance law for any reframing of the reference configuration: This should give a material form of Doyle-Ericksen formula for Eshelby's stress tensor.

(iv) Spatial energy balance for any reframing of the reference configuration: This should give a spatial form of Doyle-Ericksen formula for Eshelby's stress tensor.

Note that cases (i) and (ii) and also cases (iii) and (iv) are equivalent as the important thing here is the type of the diffeomorphism.

\section{B. Revisiting Marsden and Hughes' theorem}

Let us first revisit Marsden and Hughes' covariant energy balance theory. ${ }^{28}$ These authors postulate a covariant spatial energy balance, i.e., they consider a motion $\varphi_{t}: \mathcal{B} \rightarrow \mathcal{S}$ and postulate that balance of energy still holds for any spatial change of frame. Marsden and Hughes consider arbitrary changes of frame for the deformed configuration and postulate that energy balance is invariant under these framings. For a given nice subset $\mathcal{U} \subset \mathcal{B}$, the (spatial) balance of energy reads

$$
\frac{d}{\mathrm{~d} t} \int_{\varphi_{t}(\mathcal{U})} \rho\left(e+\frac{1}{2}\langle\langle\mathbf{v}, \mathbf{v}\rangle\rangle\right) \mathrm{d} v=\int_{\varphi_{t}(\mathcal{U})} \rho(\langle\langle\mathbf{b}, \mathbf{v}\rangle\rangle+r) \mathrm{d} v+\int_{\partial \varphi_{t}(\mathcal{U})}(\langle\langle\mathbf{t}, \mathbf{v}\rangle\rangle+h) \mathrm{d} a,
$$

where $e, r$, and $h$ are the internal energy function per unit mass, the heat supply per unit mass and the heat flux, respectively. Marsden and Hughes then consider an arbitrary reframing of the deformed configuration, which can be regarded as a motion of $\mathcal{S}$ in $\mathcal{S}$, i.e., $\xi_{t}: \mathcal{S} \rightarrow \mathcal{S}$. Postulating 
the balance of energy (5.13) for such a reframing and considering it for $t=t_{0}$ they obtain (i) conservation of mass, (ii) balance of linear momentum, (iii) balance of angular momentum, and (iv) the Doyle-Ericksen formula. Conversely, if (i), (ii), (iii), (iv) and balance of energy hold, then balance of energy would hold for any change of spatial frame. We will give a proof for the converse of the theorem in the sequel.

Proposition 5.2 (Transport theorem in a reframing of the deformed configuration): Suppose $f^{\prime}=\xi_{t^{*}} f$ is a scalar quantity defined on $\varphi_{t}^{\prime}(\mathcal{U})$, i.e., $f^{\prime}: \varphi_{t}^{\prime}(\mathcal{U}) \rightarrow \mathbb{R}$ and $f: \varphi_{t}(\mathcal{U}) \rightarrow \mathbb{R}$. [Marsden and Hughes have the following transport theorem on p. 166 of Ref. 28 in the second equation after their Eq. (2), which needs to be corrected:

$$
\frac{d}{\mathrm{~d} t} \int_{\varphi_{t}^{\prime}(\mathcal{U})} f \mathrm{~d} v=\int_{\varphi_{t}^{\prime}(\mathcal{U})}(\dot{f}+f \operatorname{div} \mathbf{v}) \mathrm{d} v^{\prime} .
$$

In fact, the first $\mathrm{d} v$ should read $\left.\mathrm{d} v^{\prime}.\right]$ Then,

$$
\left.\frac{d}{\mathrm{~d} t}\right|_{t=t_{0}} \int_{\varphi_{t}^{\prime}(\mathcal{U})} f^{\prime} \mathrm{d} v^{\prime}=\int_{\varphi_{t}(\mathcal{U})}(\dot{f}+f \operatorname{div} \mathbf{v}) \mathrm{d} v .
$$

Proof: The usual transport theorem can be written as

$$
\frac{d}{\mathrm{~d} t} \int_{\varphi_{t}^{\prime}(\mathcal{U})} f^{\prime} \mathrm{d} v^{\prime}=\int_{\varphi_{t}^{\prime}(\mathcal{U})}\left(\dot{f}^{\prime}+f^{\prime} \operatorname{div}^{\prime} \mathbf{v}^{\prime}\right) \mathrm{d} v^{\prime},
$$

where

$$
\dot{f}^{\prime}=\frac{\partial f^{\prime}}{\partial t}+\frac{\partial f^{\prime}}{\partial \mathbf{x}^{\prime}} \cdot \mathbf{v}^{\prime}=\frac{\partial f^{\prime}}{\partial t}+\mathrm{d} f^{\prime} \cdot \mathbf{v}^{\prime}
$$

and

$$
\mathbf{v}^{\prime}=\xi_{t} * \mathbf{v}+\mathbf{w}
$$

Therefore,

$$
\frac{d}{\mathrm{~d} t} \int_{\varphi_{t}^{\prime}(\mathcal{U})} f^{\prime} \mathrm{d} v^{\prime}=\int_{\varphi_{t}^{\prime}(\mathcal{U})}\left[\frac{\partial f^{\prime}}{\partial t}+\mathrm{d} f^{\prime} \cdot\left(\xi_{t^{*}} \mathbf{v}+\mathbf{w}\right)+f^{\prime} \operatorname{div}^{\prime} \mathbf{v}^{\prime}\right] \mathrm{d} v^{\prime} .
$$

Note that

$$
\frac{\partial}{\partial \mathbf{x}}=\frac{\partial}{\partial \mathbf{x}^{\prime}} \circ\left(T \xi_{t}\right) \quad \text { or } \quad \frac{\partial}{\partial \mathbf{x}^{\prime}}=\left(T \xi_{t}\right)^{-1} \circ \frac{\partial}{\partial \mathbf{x}}
$$

This means that

$$
\left.\frac{\partial}{\partial \mathbf{x}^{\prime}}\right|_{t=t_{0}}=\frac{\partial}{\partial \mathbf{x}} .
$$

Lemma 5.3: If $\xi_{t}: \mathcal{S} \rightarrow \mathcal{S}$ is a diffeomorphism with the properties,

$$
\left.\xi_{t}\right|_{t=t_{0}}=\mathrm{Id},\left.\quad T \xi_{t}\right|_{t=t_{0}}=\mathrm{Id} .
$$

Then

$$
\left.\left(\operatorname{div}^{\prime} \mathbf{v}^{\prime} \mathrm{d} v^{\prime}\right)\right|_{t=t_{0}}=\operatorname{div} \mathbf{v} \mathrm{d} v .
$$

Proof: We prove the lemma when $\mathcal{S}$ is equipped with an arbitrary volume form $\mu$. This will imply the particular case of a Riemannian manifold with the volume form induced by the Rie- 
mannian metric. Recall that the divergence of a vector field $\mathbf{X}$ with respect to $\mu$ is defined as

$$
\mathbf{L}_{\mathbf{X}} \mu=\left(\operatorname{div}_{\mu} \mathbf{X}\right) \mu .
$$

Under the spatial change of frame $\mathbf{v}^{\prime}=\xi_{t^{*}} \mathbf{X}+\mathbf{w}, \mu^{\prime}=\xi_{t^{*}} \mu$. Thus,

$$
\left(\operatorname{div}_{\mu^{\prime}} \mathbf{v}^{\prime}\right) \mu^{\prime}=\mathbf{L}_{\mathbf{v}^{\prime}}\left(\xi_{t^{*}} \mu\right)=\xi_{t^{*}}\left(\mathbf{L}_{\mathbf{v}} \mu\right),
$$

where use was made of Theorem 6.19 of Marsden and Hughes ${ }^{28}$. Therefore,

$$
\left.\left(\operatorname{div}^{\prime} \mathbf{v}^{\prime} \mathrm{d} v^{\prime}\right)\right|_{t=t_{0}}=\operatorname{div} \mathbf{v} \mathrm{d} v \text {. }
$$

One should be careful with partial time derivatives as $\partial f^{\prime} / \partial t$ is not equal to $\partial f / \partial t$ at $t=t_{0}$ because the former is partial time derivative for fixed $\mathbf{x}^{\prime}$ while the latter is a partial time derivative for fixed $\mathbf{x}$. Note that

$$
\left.\frac{\partial f^{\prime}}{\partial t}\right|_{\mathbf{x} \text { fixed }}=\left.\frac{\partial f^{\prime}}{\partial t}\right|_{\mathbf{x}^{\prime} \text { fixed }}+\mathrm{d} f^{\prime} \cdot \mathbf{w}_{t}
$$

Hence,

$$
\left(\left.\frac{\partial f^{\prime}}{\partial t}\right|_{\mathbf{x}^{\prime} \text { fixed }}\right)_{t=t_{0}}=\frac{\partial f}{\partial t}-\mathrm{d} f \cdot \mathbf{w} .
$$

Therefore (5.19) is simplified to

$$
\left.\frac{d}{\mathrm{~d} t}\right|_{t=t_{0}} \int_{\varphi_{t}^{\prime}(\mathcal{U})} f^{\prime} \mathrm{d} v^{\prime}=\int_{\varphi_{t}(\mathcal{U})}(\dot{f}+f \operatorname{div} \mathbf{v}) \mathrm{d} v .
$$

Now let us take a more natural approach and assume that we are transporting a 3-form. Note that this is more general in the sense that we have not chosen a volume form $\mathrm{d} v$ a priori.

Proposition 5.4: Suppose $\alpha^{\prime}=\xi_{t^{*}} \alpha$ is a 3-form defined on $\varphi_{t}^{\prime}(\mathcal{U})$. Then,

$$
\left.\frac{d}{\mathrm{~d} t}\right|_{t=t_{0}} \int_{\varphi_{t}^{\prime}(\mathcal{U})} \alpha^{\prime}=\int_{\varphi_{t}(\mathcal{U})} \mathbf{L}_{\mathbf{v}} \alpha .
$$

Proof: Using the usual transport theorem for forms we have

$$
\frac{d}{\mathrm{~d} t} \int_{\varphi_{t}^{\prime}(\mathcal{U})} \alpha^{\prime}=\int_{\varphi_{t}^{\prime}(\mathcal{U})} \mathbf{L}_{\mathbf{v}^{\prime}} \alpha^{\prime} .
$$

Assuming that $\alpha$ transforms objectively, i.e., $\alpha^{\prime}=\xi_{t^{*}} \alpha$, using Theorem 6.19 of Marsden and Hughes $^{28}$ we have

$$
\mathbf{L}_{\mathbf{v}^{\prime}} \alpha^{\prime}=\xi_{t^{*}} \mathbf{L}_{\mathbf{v}} \alpha
$$

Thus,

$$
\frac{d}{\mathrm{~d} t} \int_{\varphi_{t}^{\prime}(\mathcal{U})} \alpha^{\prime}=\int_{\varphi_{t}^{\prime}(\mathcal{U})} \xi_{t^{*}} \mathbf{L}_{\mathbf{v}} \alpha .
$$

Therefore, 


$$
\left.\frac{d}{\mathrm{~d} t}\right|_{t=t_{0}} \int_{\varphi_{t}^{\prime}(\mathcal{U})} \alpha^{\prime}=\int_{\varphi_{t}(\mathcal{U})} \mathbf{L}_{\mathbf{v}} \alpha .
$$

Now substitute $\alpha=f \mathrm{~d} v$, where $f$ is a scalar. Note that

$$
f^{\prime} \mathrm{d} v^{\prime}=f^{\prime} \wedge \mathrm{d} v^{\prime}=\left(\xi_{t} *\right) \wedge\left(\xi_{t^{*}} \mathrm{~d} v\right)=\xi_{t^{*}}(f \wedge \mathrm{d} v)=\xi_{t^{*}}(f \mathrm{~d} v) .
$$

The above proposition now reads

$$
\left.\frac{d}{\mathrm{~d} t}\right|_{t=t_{0}} \int_{\varphi_{t}^{\prime}(\mathcal{U})} f^{\prime} \mathrm{d} v^{\prime}=\int_{\varphi_{t_{0}}(\mathcal{U})} \mathbf{L}_{\mathbf{v}}(f \mathrm{~d} v) .
$$

Note that $\mathbf{L}$ is a derivation and hence

$$
\mathbf{L}_{\mathbf{v}}(f \mathrm{~d} v)=\left(\mathbf{L}_{\mathbf{v}} f\right) \mathrm{d} v+f\left(\mathbf{L}_{\mathbf{v}} \mathrm{d} v\right)=(\dot{f}+\operatorname{div} \mathbf{v}) \mathrm{d} v .
$$

Therefore

$$
\left.\frac{d}{\mathrm{~d} t}\right|_{t=t_{0}} \int_{\varphi_{t}^{\prime}(\mathcal{U})} f^{\prime} d v^{\prime}=\int_{\varphi_{t_{0}}(\mathcal{U})}(\dot{f}+f \operatorname{div} \mathbf{v}) \mathrm{d} v .
$$

Thus, this approach recovers the same transport equation (5.15).

\section{Energy balance in terms of differential forms}

In this section we regard $\rho$ as a 3 -form and write the energy balance equation as

$$
\frac{d}{\mathrm{~d} t} \int_{\varphi_{t}(\mathcal{U})} \rho\left(e+\frac{1}{2}\langle\langle\mathbf{v}, \mathbf{v}\rangle\rangle\right)=\int_{\varphi_{t}(\mathcal{U})} \rho(\langle\langle\mathbf{b}, \mathbf{v}\rangle\rangle+r)+\int_{\partial \varphi_{t}(\mathcal{U})}(\langle\langle\mathbf{t}, \mathbf{v}\rangle\rangle+h) \mathrm{d} a .
$$

[Traction can be thought of as a covector-valued 2-form. There are some technical details involved and we choose to stick to the usual definition of traction.] Under a spatial diffeomorphism $\xi_{t}: \mathcal{S}$ $\rightarrow \mathcal{S}$ we postulate that

$$
\frac{d}{\mathrm{~d} t} \int_{\varphi_{t}^{\prime}(\mathcal{U})} \rho^{\prime}\left(e^{\prime}+\frac{1}{2}\left\langle\left\langle\mathbf{v}^{\prime}, \mathbf{v}^{\prime}\right\rangle\right\rangle\right)=\int_{\varphi_{t}^{\prime}(\mathcal{U})} \rho^{\prime}\left(\left\langle\left\langle\mathbf{b}^{\prime}, \mathbf{v}^{\prime}\right\rangle\right\rangle+r^{\prime}\right)+\int_{\partial \varphi_{t}^{\prime}(\mathcal{U})}\left(\left\langle\left\langle\mathbf{t}^{\prime}, \mathbf{v}^{\prime}\right\rangle\right\rangle+h^{\prime}\right) \mathrm{d} a^{\prime} .
$$

Let $f$ be the scalar multiplying the density 3 -form in the first integrand, i.e., $f:=e+\frac{1}{2}\langle\langle\mathbf{v}, \mathbf{v}\rangle\rangle$. Thus

$$
\frac{d}{\mathrm{~d} t} \int_{\varphi_{t}(\mathcal{U})} \rho f=\int_{\varphi_{t}(\mathcal{U})} \mathbf{L}_{\mathbf{v}}(\rho f)=\int_{\varphi_{t}(\mathcal{U})}\left(\rho \mathbf{L}_{\mathbf{v}} f+f \mathbf{L}_{\mathbf{v}} \rho\right) .
$$

But

$$
\begin{aligned}
\mathbf{L}_{\mathbf{v}} f & =\mathbf{L}_{\mathbf{v}} e+\mathbf{L}_{\mathbf{v}}\left(\frac{1}{2}\langle\langle\mathbf{v}, \mathbf{v}\rangle\rangle\right)=\dot{e}+\frac{\partial}{\partial t}\left(\frac{1}{2}\langle\langle\mathbf{v}, \mathbf{v}\rangle\rangle\right)+d\left(\frac{1}{2}\langle\langle\mathbf{v}, \mathbf{v}\rangle\rangle\right) \cdot \mathbf{v} \\
& =\dot{e}+\left\langle\left\langle\frac{\partial \mathbf{v}}{\partial t}, \mathbf{v} \|\right)+\left\langle\left\langle\mathbf{v}, \nabla_{\mathbf{v}} \mathbf{v}\right\rangle\right\rangle=\dot{e}+\langle\langle\mathbf{v}, \mathbf{a}\rangle\rangle .\right.
\end{aligned}
$$

Also, 


$$
\frac{d}{\mathrm{~d} t} \int_{\varphi_{t}^{\prime}(\mathcal{U})} \rho^{\prime} f^{\prime}=\int_{\varphi_{t}(\mathcal{U})} \mathbf{L}_{\mathbf{v}^{\prime}}\left(\rho^{\prime} f^{\prime}\right)=\int_{\varphi_{t}^{\prime}(\mathcal{U})}\left(\rho^{\prime} \mathbf{L}_{\mathbf{v}^{\prime}} f^{\prime}+f^{\prime} \mathbf{L}_{\mathbf{v}^{\prime}} \rho^{\prime}\right) .
$$

Note that $\mathbf{v}^{\prime}=\xi_{t} * \mathbf{v}+\mathbf{w}_{t}$ and thus

$$
\mathbf{L}_{\mathbf{v}^{\prime}} \rho^{\prime}=\xi_{t^{*}}\left(\mathbf{L}_{\mathbf{v}} \rho\right) .
$$

Also,

$$
\mathbf{L}_{\mathbf{v}^{\prime}} f^{\prime}=\dot{e^{\prime}}+\left\langle\left\langle\mathbf{v}^{\prime}, \frac{\partial \mathbf{v}^{\prime}}{\partial t}+\nabla_{\mathbf{v}^{\prime}} \mathbf{v}^{\prime}\right\rangle\right)=\dot{e^{\prime}}+\left\langle\left\langle\mathbf{v}^{\prime}, \mathbf{a}^{\prime}\right\rangle\right\rangle .
$$

Thus

$$
\begin{aligned}
&\left.\left(\mathbf{L}_{\mathbf{v}^{\prime}} f^{\prime}\right)\right|_{t=t_{0}}= \dot{e}+\frac{\partial e}{\partial \mathbf{g}}: \mathfrak{L}_{\mathbf{w}} \mathbf{g}+\left\langle\left\langle\mathbf{v}+\mathbf{w},\left.\mathbf{a}^{\prime}\right|_{t=t_{0}}\right\rangle\right\rangle, \\
&\left.\left(\mathbf{L}_{\mathbf{v}^{\prime}} \boldsymbol{\rho}^{\prime}\right)\right|_{t=t_{0}}=\mathbf{L}_{\mathbf{v}} \rho .
\end{aligned}
$$

Therefore

$$
\begin{aligned}
\left.\frac{d}{\mathrm{~d} t}\right|_{t=t_{0}} \int_{\varphi_{t}^{\prime}(\mathcal{U})} \rho^{\prime} f^{\prime}= & \int_{\varphi_{t}(\mathcal{U})} \rho\left(\dot{e}+\frac{\partial e}{\partial \mathbf{g}}: \mathfrak{L}_{\mathbf{w}} \mathbf{g}+\left\langle\left\langle\mathbf{v}+\mathbf{w},\left.\mathbf{a}^{\prime}\right|_{t=t_{0}}\right\rangle\right\rangle\right) \\
& +\int_{\varphi_{t}(\mathcal{U})}\left(f+\langle\langle\mathbf{v}, \mathbf{w}\rangle\rangle+\frac{1}{2}\langle\langle\mathbf{w}, \mathbf{w}\rangle\rangle\right) \mathbf{L}_{\mathbf{v}} \rho .
\end{aligned}
$$

Now subtracting the balance of energy equation for $\varphi_{t}(\mathcal{U})$ from that of $\varphi_{t}^{\prime}(\mathcal{U})$ at $t=t_{0}$ we obtain

$$
\begin{aligned}
& \int_{\varphi_{t}(\mathcal{U})} \rho\left(\frac{\partial e}{\partial \mathbf{g}}: \mathcal{L}_{\mathbf{w}} \mathbf{g}+\left\langle\left\langle\mathbf{v},\left.\mathbf{a}^{\prime}\right|_{t=t_{0}}-\mathbf{a}\right\rangle\right\rangle+\left\langle\left\langle\mathbf{w},\left.\mathbf{a}^{\prime}\right|_{t=t_{0}}\right\rangle\right)+\int_{\varphi_{t}(\mathcal{U})}\left(\langle\langle\mathbf{v}, \mathbf{w}\rangle\rangle+\frac{1}{2}\langle\langle\mathbf{w}, \mathbf{w}\rangle\rangle\right) \mathbf{L}_{\mathbf{v}} \rho\right. \\
& =\int_{\varphi_{t}(\mathcal{U})}\left(\left\langle\left\langle\mathbf{v},\left.\mathbf{b}^{\prime}\right|_{t=t_{0}}-\mathbf{b}+\left\langle\left\langle\mathbf{w},\left.\mathbf{b}^{\prime}\right|_{t=t_{0}}\right\rangle\right\rangle\right)+\int_{\partial \varphi_{t}(\mathcal{U})}\langle\langle\mathbf{w}, \mathbf{t}\rangle\rangle \mathrm{d} a .\right.\right.
\end{aligned}
$$

Using the identity $\left.\left(\mathbf{b}^{\prime}-\mathbf{a}^{\prime}\right)\right|_{t=t_{0}}=\mathbf{b}-\mathbf{a}$ we have

$$
\int_{\varphi_{t}(\mathcal{U})} \rho\left(\frac{\partial e}{\partial \mathbf{g}}: \mathcal{L}_{\mathbf{w}} \mathbf{g}+\langle\langle\mathbf{w}, \mathbf{a}-\mathbf{b}\rangle\rangle\right)+\int_{\varphi_{t}(\mathcal{U})}\left(\langle\langle\mathbf{v}, \mathbf{w}\rangle\rangle+\frac{1}{2}\langle\langle\mathbf{w}, \mathbf{w}\rangle\rangle\right) \mathbf{L}_{\mathbf{v}} \rho=\int_{\partial \varphi_{t}(\mathcal{U})}\langle\langle\mathbf{w}, \mathbf{t}\rangle\rangle \mathrm{d} a .
$$

We know that

$$
\int_{\partial \varphi_{t}(\mathcal{U})}\langle\langle\mathbf{w}, \mathbf{t}\rangle\rangle \mathrm{d} a=\int_{\varphi_{t}(\mathcal{U})}\left(\langle\langle\operatorname{div} \boldsymbol{\sigma}, \mathbf{w}\rangle\rangle+\boldsymbol{\sigma}: \frac{1}{2} \mathcal{L}_{\mathbf{w}} \mathbf{g}+\boldsymbol{\sigma}: \boldsymbol{\omega}\right) \mathrm{d} v,
$$

where $\boldsymbol{\omega}$ has the coordinate representation $\omega_{a b}=\frac{1}{2}\left(w_{a \mid b}-w_{b \mid a}\right)$. Let us replace $\rho$ by $\rho \mathrm{d} v$ in the first integral of Eq. (5.50), 


$$
\begin{gathered}
\int_{\varphi_{t}(\mathcal{U})} \rho\left(\frac{\partial e}{\partial \mathbf{g}}: \mathcal{L}_{\mathbf{w}} \mathbf{g}+\langle\langle\mathbf{w}, \mathbf{a}-\mathbf{b}\rangle\rangle\right) \mathrm{d} v+\int_{\varphi_{t}(\mathcal{U})}\left(\langle\langle\mathbf{v}, \mathbf{w}\rangle\rangle+\frac{1}{2}\langle\langle\mathbf{w}, \mathbf{w}\rangle\rangle\right) \mathbf{L}_{\mathbf{v}} \rho \\
=\int_{\varphi_{t}(\mathcal{U})}\left(\langle\langle\operatorname{div} \boldsymbol{\sigma}, \mathbf{w}\rangle\rangle+\boldsymbol{\sigma}: \frac{1}{2} \mathcal{L}_{\mathbf{w}} \mathbf{g}+\boldsymbol{\sigma}: \boldsymbol{\omega}\right) \mathrm{d} v .
\end{gathered}
$$

Since $\mathbf{w}$ is arbitrary we conclude that

$$
\begin{gathered}
\mathbf{L}_{\mathbf{v}} \rho=0, \\
\boldsymbol{\sigma}=2 \rho \frac{\partial e}{\partial \mathbf{g}}, \\
\operatorname{div} \boldsymbol{\sigma}+\rho \mathbf{b}=\rho \mathbf{a}, \\
\boldsymbol{\sigma}^{\top}=\boldsymbol{\sigma} .
\end{gathered}
$$

\section{Proof of the converse of Marsden and Hughes' theorem}

Marsden and Hughes ${ }^{28}$ do not give a proof for the converse of the covariant energy balance theorem, i.e., when Eqs. (5.53)-(5.56) are satisfied then energy balance is invariant under $\xi_{t}: \mathcal{S}$ $\rightarrow \mathcal{S}$. Such a proof is nontrivial and is given here.

Let us assume that Eqs. (5.53)-(5.56) are satisfied and define

$$
\Delta E\left(\xi_{t}\right)=\frac{d}{\mathrm{~d} t} \int_{\varphi_{t}^{\prime}(\mathcal{U})} \rho^{\prime}\left(e^{\prime}+\frac{1}{2}\left\langle\left\langle\mathbf{v}^{\prime}, \mathbf{v}^{\prime}\right\rangle\right\rangle_{\mathbf{g}}\right)-\int_{\varphi_{t}^{\prime}(\mathcal{U})} \rho^{\prime}\left(\left\langle\left\langle\mathbf{b}^{\prime}, \mathbf{v}^{\prime}\right\rangle\right\rangle_{\mathbf{g}}+r^{\prime}\right)-\int_{\partial \varphi_{t}^{\prime}(\mathcal{U})}\left(\left\langle\left\langle\mathbf{t}^{\prime}, \mathbf{v}^{\prime}\right\rangle\right\rangle_{\mathbf{g}}+h^{\prime}\right) \mathrm{d} a^{\prime} .
$$

Note that balance of energy for $\varphi_{t}(\mathcal{U})$ can be written as $\Delta E(\mathrm{Id})=0$. We need to prove that for any diffeomorphism $\xi_{t}, \Delta E\left(\xi_{t}\right)=0$. We know that

$$
e^{\prime}\left(x^{\prime}, t, \mathbf{g}\right)=e\left(x, t, \xi_{t}^{*}(\mathbf{g})\right) .
$$

Let us denote

$$
\mathbf{w}_{t}:=\frac{d}{\mathrm{~d} t} \xi_{t}, \quad \mathbf{W}_{t}=\xi_{t}^{*}\left(\mathbf{w}_{t}\right), \quad \mathbf{g}_{t}=\xi_{t}^{*}(\mathbf{g})
$$

Note that by definition

$$
\int_{\varphi_{t}^{\prime}(\mathcal{U})} \rho^{\prime} r^{\prime}=\int_{\varphi_{t}(\mathcal{U})} \rho r, \quad \int_{\partial \varphi_{t}^{\prime}(\mathcal{U})} h^{\prime} \mathrm{d} a^{\prime}=\int_{\partial \varphi_{t}(\mathcal{U})} h \mathrm{~d} a
$$

Also note that

$$
\int_{\partial \varphi_{t}^{\prime}(\mathcal{U})}\left\langle\left\langle\mathbf{t}^{\prime}, \mathbf{v}^{\prime}\right\rangle\right\rangle_{\mathbf{g}} \mathrm{d} a^{\prime}=\int_{\partial \varphi_{t}^{\prime}(\mathcal{U})}\left\langle\left\langle\xi_{t}: \mathbf{t}, \xi_{t^{*}} \mathbf{v}+\mathbf{w}_{t}\right\rangle\right\rangle_{\mathbf{g}} \mathrm{d} a^{\prime}=\int_{\partial \varphi_{t}(\mathcal{U})}\left\langle\left\langle\mathbf{t}, \mathbf{v}+\mathbf{W}_{t}\right\rangle\right\rangle_{\mathbf{g}_{t}} \mathrm{~d} a .
$$

A straightforward computation shows that 


$$
\begin{aligned}
\frac{d}{\mathrm{~d} t} \int_{\varphi_{t}^{\prime}(\mathcal{U})}\left(\frac{1}{2} \rho^{\prime}\left\langle\left\langle\mathbf{v}^{\prime}, \mathbf{v}^{\prime}\right\rangle\right\rangle_{\mathbf{g}}-\rho^{\prime}\left\langle\left\langle\mathbf{b}^{\prime}, \mathbf{v}^{\prime}\right\rangle\right\rangle_{\mathbf{g}}\right)= & \int_{\varphi_{t}^{\prime}(\mathcal{U})} \rho^{\prime}\left\langle\left\langle\xi_{t^{*}}(\mathbf{a}-\mathbf{b}), \xi_{t^{*}} \mathbf{v}+\mathbf{w}_{t}\right\rangle\right\rangle_{\mathbf{g}}=\int_{\varphi_{t}(\mathcal{U})} \rho\langle\langle\mathbf{a}-\mathbf{b}, \mathbf{v}\rangle\rangle_{\mathbf{g}_{t}} \\
& +\int_{\varphi_{t}(\mathcal{U})} \rho\left\langle\left\langle\mathbf{a}-\mathbf{b}, \mathbf{W}_{t}\right\rangle\right\rangle_{\mathbf{g}_{t}}
\end{aligned}
$$

where use was made of $\mathbf{L}_{\mathbf{v}} \rho=0$. Note that

$$
\frac{d}{\mathrm{~d} t} \int_{\varphi_{t}^{\prime}(\mathcal{U})} \rho^{\prime} e^{\prime}=\int_{\varphi_{t}^{\prime}(\mathcal{U})}\left[\rho^{\prime} \mathbf{L}_{\mathbf{v}^{\prime}} e^{\prime}+e^{\prime} \xi_{t^{*}}\left(\mathbf{L}_{\mathbf{v}} \rho\right)\right]=\int_{\varphi_{t}^{\prime}(\mathcal{U})} \rho^{\prime} \mathbf{L}_{\mathbf{v}^{\prime}} e^{\prime}=\int_{\varphi_{t}^{\prime}(\mathcal{U})} \rho \xi_{t}^{*}\left(\mathbf{L}_{\mathbf{v}^{\prime}} e^{\prime}\right) .
$$

But

$$
\xi_{t}^{*}\left(\mathbf{L}_{\mathbf{v}}, e^{\prime}\right)=\dot{e}+\frac{\partial e}{\partial \mathbf{g}_{t}}: \mathcal{L}_{\mathbf{W}_{t}} \mathbf{g}_{t}
$$

Therefore

$$
\begin{aligned}
\Delta E\left(\xi_{t}\right) & =\Delta E(\mathrm{Id})+\int_{\varphi_{t}(\mathcal{U})}\left[\left(2 \rho \frac{\partial e}{\partial \mathbf{g}_{t}}-\boldsymbol{\sigma}\right): \frac{1}{2} \mathcal{L}_{\mathbf{W}_{t}} \mathbf{g}_{t}+\boldsymbol{\sigma}: \boldsymbol{\omega}_{t}\right] \mathrm{d} v-\int_{\varphi_{t}(\mathcal{U})}\left\langle\left\langle\operatorname{div} \boldsymbol{\sigma}+\rho(\mathbf{b}-\mathbf{a}), \mathbf{W}_{t}\right\rangle\right\rangle_{\mathbf{g}_{t}} \mathrm{~d} v \\
& =0 .
\end{aligned}
$$

\section{E. Spatial covariant material energy balance}

Let us consider the material balance of energy

$$
\frac{d}{\mathrm{~d} t} \int_{\mathcal{U}} \rho_{0}\left(E+\frac{1}{2}\langle\langle\mathbf{V}, \mathbf{V}\rangle\rangle\right)=\int_{\mathcal{U}} \rho_{0}(\langle\langle\mathbf{B}, \mathbf{V}\rangle\rangle+R)+\int_{\partial \mathcal{U}}(\langle\langle\mathbf{T}, \mathbf{V}\rangle\rangle+H) \mathrm{d} A,
$$

where we have assumed that $\rho_{0}$ is a 3 -form. Physically this is equivalent to the spatial energy balance; material energy balance is simply the spatial energy balance expressed in terms of quantities defined with respect to the reference configuration. Let us postulate that the material energy balance is invariant with respect to diffeomorphisms $\xi_{t}: \mathcal{S} \rightarrow \mathcal{S}$. This is physically equivalent to the postulate of covariant spatial energy balance. The material energy balance for $\varphi_{t}^{\prime}(\mathcal{U}) \subset \mathcal{S}$ is written as

$$
\frac{d}{\mathrm{~d} t} \int_{\mathcal{U}} \rho_{0}^{\prime}\left(E^{\prime}+\frac{1}{2}\left\langle\left\langle\mathbf{V}^{\prime}, \mathbf{V}^{\prime}\right\rangle\right\rangle\right)=\int_{\mathcal{U}} \rho_{0}^{\prime}\left(\left\langle\left\langle\mathbf{B}^{\prime}, \mathbf{V}^{\prime}\right\rangle\right\rangle+R^{\prime}\right)+\int_{\partial \mathcal{U}}\left(\left\langle\left\langle\mathbf{T}^{\prime}, \mathbf{V}^{\prime}\right\rangle\right\rangle+H^{\prime}\right) \mathrm{d} A
$$

Note that for both deformations balance of energy is written for the same subset $\mathcal{U} \subset \mathcal{B}$. The material velocity $\mathbf{V}^{\prime}$ is related to $\mathbf{V}$ by the following relation:

$$
\mathbf{V}^{\prime}(\mathbf{X})=T \xi_{t} \circ \mathbf{V}_{t}+\mathbf{w}_{t} \circ \varphi_{t}(\mathbf{X}) .
$$

Thus

$$
\left.\mathbf{V}^{\prime}\right|_{t=t_{0}}=\mathbf{V}+\mathbf{w} \circ \varphi_{t_{0}} .
$$

We know that

$$
R=J_{\varphi_{t}} r^{\circ} \varphi_{t}, \quad R^{\prime}=J_{\varphi_{t}^{\prime}} r^{\prime} \circ \varphi_{t}^{\prime}, \quad r=J_{\xi_{t}} r^{\prime} \circ \xi_{t} .
$$

Hence 


$$
J_{\varphi_{t}^{\prime}} r^{\prime} \circ \varphi_{t}^{\prime}=\left(J_{\xi_{t}} r^{\prime} \circ \xi_{t}\right) \circ \varphi_{t} J_{\varphi_{t}}=J_{\varphi_{t}} r^{\circ} \varphi_{t}
$$

Thus

$$
R^{\prime}=R
$$

Similarly

$$
H^{\prime}=H \text {. }
$$

Note that looking at densities as 3-forms

$$
\rho_{0}(\mathbf{X}, t)=\varphi_{t}^{*} \rho(\mathbf{x}, t), \quad \rho_{0}^{\prime}(\mathbf{X}, t)=\left(\varphi_{t}^{\prime}\right)^{*} \rho^{\prime}\left(\mathbf{x}^{\prime}, t\right) .
$$

But

$$
\left(\varphi_{t}^{\prime}\right)^{*} \rho^{\prime}\left(\mathbf{x}^{\prime}, t\right)=\left(\xi_{t} \circ \varphi_{t}\right)^{*} \rho^{\prime}\left(\mathbf{x}^{\prime}, t\right)=\left(\varphi_{t}^{*} \circ \xi_{t}^{*}\right) \circ \xi_{t^{*}} \rho(\mathbf{x}, t)=\varphi_{t}^{*} \rho(\mathbf{x}, t)
$$

Thus

$$
\rho_{0}^{\prime}(\mathbf{X}, t)=\rho_{0}(\mathbf{X}, t) .
$$

Because balance of energy is written for the same subset $\mathcal{U} \subset \mathcal{B}$ the same equality holds for densities as scalar fields, i.e., one can replace $\rho_{0}^{\prime}$ and $\rho_{0}$ by $\rho_{0}^{\prime} \mathrm{d} V$ and $\rho_{0} \mathrm{~d} V$, respectively. Define

$$
E(\mathbf{X}, t, \mathbf{g})=e\left(\varphi_{t}(\mathbf{X}), t, \mathbf{g} \circ \varphi_{t}(\mathbf{X})\right)
$$

We know that

$$
e^{\prime}\left(\mathbf{x}^{\prime}, t, \mathbf{g}\right)=e\left(\mathbf{x}, t, \xi_{t}^{*} \mathbf{g}\right)
$$

Thus

$$
E^{\prime}(\mathbf{X}, t, \mathbf{g})=e^{\prime}\left(\mathbf{x}^{\prime}, t, \mathbf{g}\right)=e\left(\mathbf{x}, t, \xi_{t}^{*} \mathbf{g}\right)=E\left(\mathbf{X}, t, \xi_{t}^{*} \mathbf{g}\right)
$$

Therefore

$$
\left.\frac{d}{\mathrm{~d} t}\right|_{t=t_{0}} E^{\prime}=\frac{\partial E}{\partial t}+\frac{\partial E}{\partial \mathbf{g}}: \mathfrak{L}_{\mathbf{w}^{\circ} \varphi_{t}}\left(\mathbf{g} \circ \varphi_{t}\right) .
$$

Now the material energy balance for the motion $\varphi_{t}^{\prime}$ at $t=t_{0}$ can be written as

$$
\begin{aligned}
\int_{\mathcal{U}} \frac{\partial \rho_{0}}{\partial t}\left(E+\frac{1}{2}\left\langle\left\langle\mathbf{V}+\mathbf{w} \circ \varphi_{t_{0}}, \mathbf{V}+\mathbf{w} \circ \varphi_{t_{0}}\right\rangle\right\rangle\right) \mathrm{d} V+\int_{\mathcal{U}}\left[\rho_{0}\left(\frac{\partial E}{\partial t}+\frac{\partial E}{\partial \mathbf{g}}: \mathfrak{L}_{\mathbf{w}^{\circ} \varphi_{t}}\left(\mathbf{g} \circ \varphi_{t}\right)\right)\right. \\
\left.\quad+\rho_{0}\left\langle\left\langle\mathbf{V}+\mathbf{w} \circ \varphi_{t_{0}},\left.\mathbf{A}^{\prime}\right|_{t=t_{0}}\right\rangle\right\rangle\right] \mathrm{d} V \\
=\int_{\mathcal{U}} \rho_{0}\left(\left\langle\left\langle\left.\mathbf{B}^{\prime}\right|_{t=t}, \mathbf{V}+\mathbf{w} \circ \varphi_{t_{0}}\right\rangle\right\rangle+R\right) \mathrm{d} V+\int_{\partial \mathcal{U}}\left(\left\langle\left\langle\mathbf{T}, \mathbf{V}+\mathbf{w} \circ \varphi_{t_{0}}\right\rangle\right\rangle+H\right) \mathrm{d} A .
\end{aligned}
$$

Subtracting the balance of energy for the motion $\varphi_{t}$ from (5.81) one arrives at the following identity: 


$$
\begin{gathered}
\int_{\mathcal{U}} \frac{\partial \rho_{0}}{\partial t}\left(\left\langle\left\langle\mathbf{V}, \mathbf{w} \circ \varphi_{t_{0}}\right\rangle\right\rangle+\frac{1}{2}\left\langle\left\langle\mathbf{w} \circ \varphi_{t_{0}}, \mathbf{w} \circ \varphi_{t_{0}}\right\rangle\right\rangle\right) \mathrm{d} V+\int_{\mathcal{U}} \rho_{0}\left(\frac{\partial E}{\partial \mathbf{g}}: \mathfrak{L}_{\mathbf{w} \circ \varphi_{t}}\left(\mathbf{g} \circ \varphi_{t}\right)+\left\langle\left\langle\mathbf{w} \circ \varphi_{t_{0}}, \mathbf{A}\right\rangle\right\rangle\right) \mathrm{d} V \\
=\int_{\mathcal{U}}\left\langle\left\langle\rho_{0} \mathbf{B}, \mathbf{w} \circ \varphi_{t_{0}}\right\rangle\right\rangle \mathrm{d} V+\int_{\partial \mathcal{U}}\left\langle\left\langle\mathbf{T}, \mathbf{w} \circ \varphi_{t_{0}}\right\rangle\right\rangle \mathrm{d} A
\end{gathered}
$$

Let us denote $\mathbf{W}=\mathbf{W}^{\circ} \varphi_{t}$ and note that $\mathbf{W}$ is a spatial vector field with components $W^{a}$.

Lemma 5.5: The surface integral term in (5.82) is transformed to a volume integral as

$$
\int_{\partial \mathcal{U}}\langle\langle\mathbf{T}, \mathbf{W}\rangle\rangle \mathrm{d} A=\int_{\mathcal{U}}(\langle\langle\text { Div } \mathbf{P}, \mathbf{W}\rangle\rangle+\tau: \boldsymbol{\omega}+\boldsymbol{\tau}: \mathbf{k}) \mathrm{d} V
$$

where, $\tau^{a b}=P^{a B} F_{B}^{b}$ is the Kirchhoff stress and $\boldsymbol{\omega}$ and $\mathbf{k}$ have the coordinate representations $k_{a b}$ $=\frac{1}{2}\left(W_{a \mid b}+W_{b \mid a}\right)$ and $\omega_{a b}=\frac{1}{2}\left(W_{a \mid b}-W_{b \mid a}\right)$.

Proof: The integrand has the following component form:

$$
T^{a} g_{a b} W^{b}=P^{a C} G_{C D} N^{D} g_{a b} W^{b}=\left(P^{a C} g_{a b} W^{b}\right) G_{C D} N^{D} .
$$

Now using divergence theorem the surface integral is transformed to an integral on $\mathcal{U}$ with an integrand with the following component form:

$$
\left(P^{a C} g_{a b} W^{b}\right)_{\mid C}=P^{a C}{ }_{\mid C} g_{a b} W^{b}+P^{a C} g_{a b} W^{b}{ }_{\mid C},
$$

where use was made of the fact that $g_{a b \mid C}=0$. Note that

$$
W^{b}{ }_{\mid C}=\frac{\partial W^{b}}{\partial X^{C}}+\gamma_{c d}^{b} W^{c} F^{d}{ }_{C}=W^{b}{ }_{, d} F_{C}^{d}+\gamma_{c d}^{b} W^{c} F^{d}{ }_{C}=\left(W^{b}{ }_{d}+\gamma_{c d}^{b} W^{c}\right) F_{C}^{d}=W_{\mid d}^{b} F_{C}^{d} .
$$

Therefore,

$$
\begin{aligned}
\left(P^{a C} g_{a b} W^{b}\right)_{\mid C} & =P^{a C}{ }_{\mid C} g_{a b} W^{b}+P^{a C} W_{a \mid d} F_{C}^{d} \\
& =P^{a C}{ }_{\mid C} g_{a b} W^{b}+P^{a C} F^{d}{ }_{C}\left[\frac{1}{2}\left(W_{a \mid d}+W_{d \mid a}\right)+\frac{1}{2}\left(W_{a \mid d}-W_{d \mid a}\right)\right],
\end{aligned}
$$

which proves the lemma.

Substituting (5.83) into (5.82) yields

$$
\begin{gathered}
\int_{\mathcal{U}} \frac{\partial \rho_{0}}{\partial t}\left(\langle\langle\mathbf{V}, \mathbf{W}\rangle\rangle+\frac{1}{2}\langle\langle\mathbf{W}, \mathbf{W}\rangle\rangle\right) \mathrm{d} V+\int_{\mathcal{U}}\left(2 \rho_{0} \frac{\partial E}{\partial \mathbf{g}}-\boldsymbol{\tau}\right): \mathbf{k} \mathrm{d} \mathbf{V}-\int_{\mathcal{U}} \boldsymbol{\tau}: \boldsymbol{\omega} \mathrm{d} \mathbf{V} \\
-\int_{\mathcal{U}}\left\langle\left\langle\text { Div } \mathbf{P}+\rho_{0} \mathbf{B}-\rho_{0} \mathbf{A}, \mathbf{W}\right\rangle\right\rangle \mathrm{d} \mathbf{V}=\mathbf{0} .
\end{gathered}
$$

As $\mathbf{W}$ and $\mathcal{U} \subset \mathcal{B}$ are arbitrary we conclude that,

$$
\begin{gathered}
\frac{\partial \rho_{0}}{\partial t}=0, \\
\tau=2 \rho_{0} \frac{\partial E}{\partial \mathbf{g}}, \\
\tau^{\top}=\tau,
\end{gathered}
$$




$$
\operatorname{Div} \mathbf{P}+\rho_{0} \mathbf{B}=\rho_{0} \mathbf{A} .
$$

In conclusion, these computations result in the following proposition.

Proposition 5.6: Energy balance written in material form, but still with the assumption of spatial covariance yields results that are identical to those of energy balance written in spatial form, also with covariance under spatial diffeomorphisms.

The converse can be proved similar to what was done in the previous subsection.

\section{TRANSFORMATION OF ENERGY BALANCE UNDER MATERIAL DIFFEOMORPHISMS}

As was seen in the preceding section, invariance of balance of energy under an arbitrary change in spatial frame is equivalent to (1) balance of linear momentum, (2) balance of angular momentum, (3) conservation of mass, and (4) Doyle-Ericksen formula. To our best knowledge, there is no material version of this theorem in the literature. Our motivation for studying the possibility of material invariance of energy balance was to gain a better understanding of configurational forces as they are believed to be related to rearrangements of the reference configuration. It turns out that, in general, energy balance cannot be invariant under diffeomorphisms of the reference configuration and what one should be looking for instead is the way in which energy balance transforms under material diffeomorphisms. In this section we first obtain such a transformation formula under an arbitrary time-dependent material diffeomorphism [see Eq. (6.51)] and then obtain the conditions under which balance of energy is materially covariant.

\section{A. The energy balance material transformation formula}

We begin with a discussion of how energy balance transforms under material diffeomorphisms. Define

$$
E(\mathbf{X}, t, \mathbf{G})=\Psi\left(\mathbf{X}, t, \mathbf{C}\left(\mathbf{F}(\mathbf{X}), \mathbf{g}\left(\varphi_{t}(\mathbf{X})\right)\right), \mathbf{G}\right),
$$

where $\Psi=\Psi(\mathbf{X}, t, \mathbf{G}, \mathbf{C})$ is the material free energy density. Material (Lagrangian) energy balance can be written as

$$
\frac{d}{\mathrm{~d} t} \int_{\mathcal{U}} \rho_{0}\left(E+\frac{1}{2}\langle\langle\mathbf{V}, \mathbf{V}\rangle\rangle\right) \mathrm{d} V=\int_{\mathcal{U}} \rho_{0}(\langle\langle\mathbf{B}, \mathbf{V}\rangle\rangle+R) \mathrm{d} V+\int_{\mathcal{U}}(\langle\langle\mathbf{T}, \mathbf{V}\rangle\rangle+H) \mathrm{d} A,
$$

which can be simplified to read

$$
\int_{\mathcal{U}} \frac{d}{\mathrm{~d} t}\left[\rho_{0}\left(E+\frac{1}{2}\langle\langle\mathbf{V}, \mathbf{V}\rangle\rangle\right)\right] \mathrm{d} V=\int_{\mathcal{U}} \rho_{0}(\langle\langle\mathbf{B}, \mathbf{V}\rangle\rangle+R) \mathrm{d} V+\int_{\partial \mathcal{U}}(\langle\langle\mathbf{T}, \mathbf{V}\rangle\rangle+H) \mathrm{d} A,
$$

where $\mathcal{U}$ is an arbitrary nice subset of the reference configuration $\mathcal{B}, \mathbf{B}$ is body force per unit undeformed mass, $\mathbf{V}(\mathbf{X}, t)$ is the material velocity, $\rho_{0}(\mathbf{X}, t)$ is the material density, $R(\mathbf{X}, t)$ is the heat supply per unit undeformed mass, and $H(\mathbf{X}, t, \hat{\mathbf{N}})$ is the heat flux across a surface with normal $\hat{\mathbf{N}}$ in the undeformed configuration (normal to $\partial \mathcal{U}$ at $\mathbf{X} \in \partial \mathcal{U}$ ). It is to be noted that this is balance of energy for a deformed part of the body written in terms of quantities that are defined with respect to the undeformed (reference) configuration. Here we assume that we have a material manifold which is a Riemannian manifold $(\mathfrak{B}, \mathbf{G})$ and a given reference configuration $\mathcal{B} \subset \mathfrak{B}$.

Change of reference frame: In this paragraph we consider a change of frame for the reference configuration and look at the transformed quantities for the new reference configuration. A reframing of the reference configuration is a diffeomorphism

$$
\Xi_{t}:(\mathfrak{B}, \mathbf{G}) \rightarrow\left(\mathfrak{B}, \mathbf{G}^{\prime}\right) .
$$

A change of frame can be thought of as a change of coordinates in the reference configuration (passive definition) or a rearrangement of microstructure (active definition). Under such a framing, a nice subset $\mathcal{U}$ is mapped to another nice subset $\mathcal{U}^{\prime}=\Xi_{t}(\mathcal{U})$ and a material point $\mathbf{X}$ is mapped to 


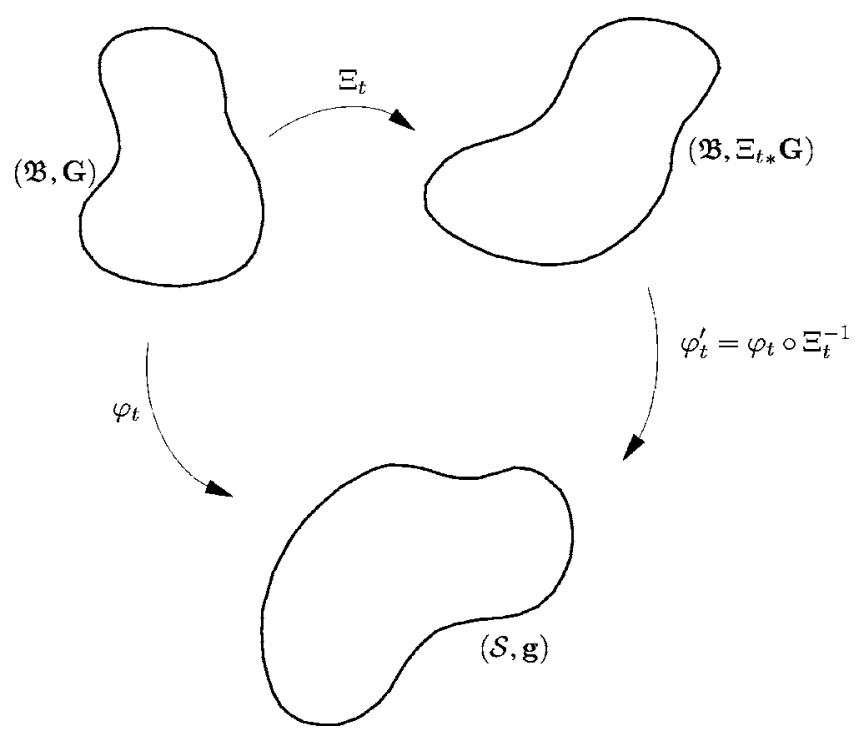

FIG. 2. A material reframing and the corresponding deformation maps.

$\mathbf{X}^{\prime}=\Xi_{t}(\mathbf{X})$. Note that $\mathbf{X}$ is the position of a particle in the reference configuration, i.e., material points are identified with their positions in the reference configuration (which is arbitrary). The change of frame is mathematically a mapping between two manifolds and one would expect to define an object on $\left(\mathfrak{B}, \mathbf{G}^{\prime}\right)$ as push-forward of the corresponding object on $(\mathfrak{B}, \mathbf{G})$.

The deformation mapping for the new reference configuration is $\varphi_{t}^{\prime}=\varphi_{t}{ }^{\circ} \Xi_{t}^{-1}$. This can be clearly seen in Fig. 2. The material velocity in $\mathcal{U}^{\prime}$ is

$$
\mathbf{V}^{\prime}\left(\mathbf{X}^{\prime}, t\right)=\frac{\partial}{\partial t} \varphi_{t}^{\prime}\left(\mathbf{X}^{\prime}\right)=\frac{\partial \varphi_{t}}{\partial t} \circ \Xi_{t}^{-1}\left(\mathbf{X}^{\prime}\right)+T \varphi_{t} \circ \frac{\partial \Xi_{t}^{-1}}{\partial t}\left(\mathbf{X}^{\prime}\right) .
$$

We assume that

$$
\left.\Xi_{t}\right|_{t=t_{0}}=\mathrm{Id}, \quad \frac{\partial \Xi_{t}}{\partial t}(\mathbf{X})=\mathbf{W}(\mathbf{X}, t) .
$$

Note that $\mathbf{W}$ is the infinitesimal generator of the rearrangement $\Xi_{t}$. It can be shown that at $t=t_{0}$,

$$
\left.\frac{\partial \Xi_{t}^{-1}}{\partial t}\left(\mathbf{X}^{\prime}\right)\right|_{t=t_{0}}=-\mathbf{W}(\mathbf{X}, t) \text {. }
$$

Thus, at $t=t_{0}$,

$$
\mathbf{V}^{\prime}=\mathbf{V}-\mathbf{F W} .
$$

To find the relation between $\mathbf{G}$ and $\mathbf{G}^{\prime}$ we note that the Finger tensor $\mathbf{b}=\varphi_{t^{*}} \mathbf{G}$ is a spatial tensor and hence independent of framing of the reference configuration. Thus,

$$
\mathbf{b}=\varphi_{t} * \mathbf{G}=\left(\varphi_{t}^{\prime}\right)_{*} \mathbf{G}^{\prime} .
$$

That is,

$$
\mathbf{G}^{\prime}=\left(\varphi_{t} \circ \Xi_{t}^{-1}\right)^{*} \circ \varphi_{t^{*}} \mathbf{G}=\left(\Xi_{t}^{-1}\right)^{*} \circ \varphi_{t}^{*} \circ \varphi_{t^{*}} \mathbf{G}=\left(\Xi_{t}^{-1}\right)^{*} \mathbf{G}=\Xi_{t^{*}} \mathbf{G}=\left(T \Xi_{t}\right)^{-*} \mathbf{G}\left(T \Xi_{t}\right)^{-1} .
$$

Note that for an arbitrary $\mathbf{X}_{0} \in \mathcal{B}$, 


$$
\mathbf{F}\left(\mathbf{X}_{0}\right): T_{\mathbf{X}_{0}} \mathcal{B} \rightarrow T_{\varphi_{t}\left(\mathbf{x}_{0}\right)} \mathcal{S} \quad \text { and } \quad \mathbf{F}^{\prime}\left(\mathbf{X}_{0}^{\prime}\right): T_{\mathbf{X}_{0}^{\prime}} \mathcal{B} \rightarrow T_{\varphi_{t}^{\prime}\left(\mathbf{X}_{0}^{\prime}\right.} \mathcal{S}
$$

Given $\mathrm{d} \mathbf{X} \in T_{\mathbf{X}_{0}} \mathcal{B}$,

$$
\mathrm{d} \mathbf{x}=\mathbf{F}\left(\mathbf{X}_{0}\right) \cdot d \mathbf{X} \quad \text { and } \quad \mathrm{d} \mathbf{X}^{\prime}=T \Xi_{t} \cdot \mathrm{d} \mathbf{X}
$$

Hence,

$$
\mathrm{d} \mathbf{x}=\mathbf{F}^{\prime}\left(\mathbf{X}_{0}^{\prime}\right) \cdot \mathrm{d} \mathbf{X}^{\prime}=\mathbf{F}^{\prime}\left(\mathbf{X}_{0}^{\prime}\right) \circ T \Xi_{t} \cdot \mathrm{d} \mathbf{X}=\mathbf{F}\left(\mathbf{X}_{0}\right) \cdot \mathrm{d} \mathbf{X}
$$

for all $\mathrm{d} \mathbf{X} \in T_{\mathbf{X}_{0}} \mathcal{B}$. Thus,

$$
\mathbf{F}^{\prime}=\Xi_{t^{*}} \mathbf{F},
$$

where

$$
\Xi_{t^{*}} \mathbf{F}=\mathbf{F} \circ\left(T \Xi_{t}\right)^{-1} .
$$

The easier way of proving this is the following:

$$
\mathbf{F}^{\prime}=T \varphi_{t}^{\prime}=T\left(\varphi_{t} \circ \Xi_{t}^{-1}\right)=T \varphi_{t} \circ\left(\Xi_{t}\right)^{-1}=\mathbf{F} \circ\left(\Xi_{t}\right)^{-1} .
$$

The material internal energy density is assumed to have the following tensorial property:

$$
E^{\prime}\left(\mathbf{X}^{\prime}, t, \mathbf{G}^{\prime}\right)=E(\mathbf{X}, t, \mathbf{G}) .
$$

Note that this is different from assuming local covariance for internal energy density. This is simply the material analogue of (5.78); all that (6.14) says is that internal energy density at $\mathbf{X}^{\prime}$ evaluated by the transformed metric $\mathbf{G}^{\prime}$ is equal to the internal energy density at $\mathbf{X}$ evaluated by the metric $\mathbf{G}$. We know that $\mathbf{G}^{\prime}=\Xi_{t^{*}} \mathbf{G}$, thus

$$
E^{\prime}\left(\mathbf{X}^{\prime}, t, \mathbf{G}\right)=E\left(\mathbf{X}, t, \Xi_{t}^{*} \mathbf{G}\right) .
$$

This means that

$$
\left.\frac{d}{\mathrm{~d} t}\right|_{t=t_{0}} E^{\prime}\left(\mathbf{X}^{\prime}, t, \mathbf{G}\right)=\frac{\partial E}{\partial t}+\frac{\partial E}{\partial \mathbf{G}}: \mathfrak{L}_{\mathbf{W}} \mathbf{G} .
$$

Remark: Marsden and Hughes ${ }^{28}$ defined covariant constitutive equations by looking at isometries of $T_{\mathbf{x}_{0}} \mathcal{B}$ at a given point $\mathbf{X}_{0} \in \mathcal{B}$. This is why they did not need to consider an explicit dependence of $\Psi$ on $\mathbf{G}$. Another more general way of defining material covariance for the strain energy function $\Psi$ is to assume that for any local diffeomorphism $\Lambda: T_{\mathbf{x}_{0}} \mathcal{B} \rightarrow T_{\Lambda\left(\mathbf{x}_{0}\right)} \Lambda(\mathcal{B})$ that leaves $\mathbf{X}_{0}$ fixed,

$$
\Psi\left(\mathbf{X}_{0}, \mathbf{G}, \mathbf{C}\right)=\Psi\left(\mathbf{X}_{0}, \Lambda^{*} \mathbf{G}, \Lambda^{*} \mathbf{C}\right) .
$$

Note that this is different from the implication of Cartan's space-time, e.g.,

$$
\Psi\left(\mathbf{X}^{\prime}, \mathbf{G}^{\prime}, \mathbf{C}^{\prime}\right)=\Psi\left(\mathbf{X}, \Xi^{*} \mathbf{G}^{\prime}, \Xi^{*} \mathbf{C}^{\prime}\right),
$$

for an arbitrary diffeomorphism $\Xi: \mathfrak{B} \rightarrow \mathfrak{B}$. We emphasize that this relation and similarly (6.14) do not put any restrictions on material properties. Ju and Papadopoulos ${ }^{26,27}$ proved that a consequence of (6.17) is the following infinitesimal covariance condition:

$$
\mathbf{G} \frac{\partial \Psi}{\partial \mathbf{G}}+\mathbf{C} \frac{\partial \Psi}{\partial \mathbf{C}}=\mathbf{0}
$$

which is equivalent to 


$$
\frac{\partial \Psi}{\partial \mathbf{G}}=-\mathbf{G}^{-1} \mathbf{C} \frac{\partial \Psi}{\partial \mathbf{C}}=-\frac{1}{2} \mathbf{G}^{-1} \mathbf{C S}=-\frac{1}{2} \mathbf{F}^{\top} \mathbf{P} .
$$

We will obtain this condition in the sequel as a consequence of assuming material covariance of energy balance.

Example: Consider a (materially uniform) Neo-Hookean material with the following energy density

$$
\Psi(\mathbf{X}, \mathbf{G}, \mathbf{C})=\mu[\operatorname{tr}(\mathbf{C})-3]=\mu\left(C_{I J} G^{I J}-3\right) .
$$

We now show that this is an example of a materially covariant material. Note that

$$
\left(\mathbf{C} \frac{\partial \Psi}{\partial \mathbf{C}}\right)_{I}^{J}=\mu C_{I K} G^{K J}
$$

Also

$$
\left(\mathbf{G} \frac{\partial \Psi}{\partial \mathbf{C}}\right)_{I}^{J}=\mu G_{I K} C_{M N} \frac{\partial G^{M N}}{\partial G_{K J}}=-\frac{\mu}{2} C_{M N} G_{I K}\left(G^{M K} G^{J N}+G^{M J} G^{K N}\right)=-\mu C_{I K} G^{K J}
$$

i.e.,

$$
\mathbf{C} \frac{\partial \Psi}{\partial \mathbf{C}}+\mathbf{G} \frac{\partial \Psi}{\partial \mathbf{G}}=\mathbf{0}
$$

Spatial covariance of strain energy function (material-frame-indifference) can be defined similarly (see Ref. 41). However, one should note that this is different from Marsden and Hughes point of departure for developing a covariant theory of elasticity; in Marsden and Hughes' theory ${ }^{28}$ balance of energy is assumed to be covariant and not the energy function. In covariant energy balance, a global diffeomorphism is considered and energy balance is assumed to be invariant under this global diffeomorphism.

Balance of energy for reframings of the reference configuration: One way to obtain the governing balance equations of a continuum is to use the homogeneity of the ambient space and postulate that if a deformed body satisfies the balance of energy, any framing of it should satisfy the balance of energy as well. This is a postulate and cannot be proved. But, one can justify it (or motivate it) by the fact that the ambient space $\mathcal{S}$ is homogeneous. Invariance of energy balance under framings of the reference configuration is less obvious and, in general, it turns out not to hold. The following is the main conclusion of this section. Under referential diffeomorphisms, material energy balance has some extra terms in it. The extra terms correspond to some forces that contribute to the rate of change of energy when the reference configuration evolves.

Consider a deformation mapping $\varphi_{t}: \mathcal{B} \rightarrow \mathcal{S}$ and a referential diffeomorphisms $\Xi_{t}: \mathfrak{B} \rightarrow \mathfrak{B}$. The mapping $\varphi_{t}^{\prime}=\varphi_{t}^{\circ} \Xi_{t}^{-1}: \mathcal{B}^{\prime} \rightarrow \mathcal{S}$, where $\mathcal{B}^{\prime}=\Xi_{t}(\mathcal{B})$, represents the deformation of the new (evolved) reference configuration. We are interested in understanding the form of material energy balance for $\Xi_{t}(\mathcal{U}) \subset \mathcal{B}^{\prime}$ for any nice $\mathcal{U} \subset \mathcal{B}$. In addition to contributions from the mapping $\varphi_{t}^{\prime}$, in general, one should expect to see contributions from the referential mapping $\Xi_{t}$ as well, i.e., evolution of reference configuration may, in general, contribute to the energy balance. Now the balance of energy should include the following two groups of terms:

(i) Looking at $\varphi_{t}^{\prime}$ as the deformation of $\mathcal{B}^{\prime}$ in $\mathcal{S}$, one has the usual material energy balance for $\Xi_{t}(\mathcal{U})$. Transformation of fields from $(\mathfrak{B}, \mathbf{G})$ to $\left(\mathfrak{B}, \mathbf{G}^{\prime}\right)$ follows Cartan's space-time theory.

(ii) Nonstandard terms may appear to represent the energy associated with the material evolution. 
Here a comment is in order. The mapping that represents all the physical processes is $\varphi_{t}$. This mapping is the composition of $\varphi_{t}^{\prime}$ and $\Xi_{t}$ and hence it is expected that, in general, both $\varphi_{t}^{\prime}$ and $\Xi_{t}$ represent part of the physical processes. This means that standard deformation represented by $\varphi_{t}^{\prime}$ and evolution of microstructure (or any other material evolution) represented by $\Xi_{t}$ should contribute to balance of energy. (This is similar to Gurtin's idea ${ }^{20,21}$ of including both standard and nonstandard terms in the expression of working. However, here we consider the full balance of energy.) This rough picture should be enough to convince the reader that the lack of invariance of energy balance under $\Xi_{t}$ should not be surprising. Lack of invariance implies the appearance of some new terms that are work-conjugate to $\mathbf{W}_{t}=(\partial / \partial t) \Xi_{t}$. Let us denote the volume and surface forces conjugate to $\mathbf{W}$ by $\mathbf{B}_{0}$ and $\mathbf{T}_{0}$, respectively.

Instead of looking at spatial framings, we fix the deformed configuration and look at framings of the reference configuration. We postulate that energy balance for each nice subset $\mathcal{U}^{\prime}$ has the following form:

$$
\begin{aligned}
\frac{d}{\mathrm{~d} t} \int_{\mathcal{U}^{\prime}}\left(E^{\prime}+\frac{1}{2} \rho_{0}^{\prime}\left\langle\left\langle\mathbf{V}^{\prime}, \mathbf{V}^{\prime}\right\rangle\right\rangle\right) \mathrm{d} V^{\prime}= & \int_{\mathcal{U}^{\prime}} \rho_{0}^{\prime}\left(\left\langle\left\langle\mathbf{B}^{\prime}, \mathbf{V}^{\prime}\right\rangle\right\rangle+R^{\prime}\right) \mathrm{d} V^{\prime}+\int_{\partial \mathcal{U}^{\prime}}\left(\left\langle\left\langle\mathbf{T}^{\prime}, \mathbf{V}^{\prime}\right\rangle\right\rangle+H^{\prime}\right) \mathrm{d} A^{\prime} \\
& +\int_{\mathcal{U}^{\prime}}\left\langle\left\langle\mathbf{B}_{0}^{\prime}, \mathbf{W}_{t}\right\rangle\right\rangle \mathrm{d} V^{\prime}+\int_{\partial \mathcal{U}^{\prime}}\left\langle\left\langle\mathbf{T}_{0}^{\prime}, \mathbf{W}_{t}\right\rangle\right\rangle \mathrm{d} A^{\prime},
\end{aligned}
$$

where $\mathcal{U}^{\prime}=\Xi_{t}(\mathcal{U})$ and $\mathbf{B}_{0}^{\prime}$ and $\mathbf{T}_{0}^{\prime}$ are unknown vector fields at this point. Using Cartan's space-time theory, it is assumed that the primed quantities have the following relations with the unprimed quantities

$$
\begin{gathered}
\mathrm{d} V^{\prime}=\Xi_{t} * \mathrm{~d} V\left(J\left(\Xi_{t}\right) \mathrm{d} V^{\prime}=\mathrm{d} V\right), \quad R^{\prime}\left(\mathbf{X}^{\prime}, t\right)=R(\mathbf{X}, t), \\
\rho_{0}^{\prime}\left(\mathbf{X}^{\prime}, t\right)=\rho_{0}(\mathbf{X}), \quad H^{\prime}\left(\mathbf{X}^{\prime}, \hat{\mathbf{N}}^{\prime}, t\right)=H(\mathbf{X}, \hat{\mathbf{N}}, t), \\
\mathbf{T}^{\prime}\left(\mathbf{X}^{\prime}, \hat{\mathbf{N}}^{\prime}, t\right)=T \Xi_{t}(\mathbf{X}) \cdot \mathbf{T}(\mathbf{X}, \hat{\mathbf{N}}, t) .
\end{gathered}
$$

We know that

$$
\mathbf{B}^{\prime}-\mathbf{A}^{\prime}=\Xi_{t^{*}}(\mathbf{B}-\mathbf{A})
$$

Thus

$$
\left.\left(\mathbf{B}^{\prime}-\mathbf{A}^{\prime}\right)\right|_{t=t_{0}}=\mathbf{B}-\mathbf{A} .
$$

Note that if $\alpha$ is a 3 -form on $\mathcal{U}$, then

$$
\left.\frac{d}{\mathrm{~d} t}\right|_{t=t_{0}} \int_{\mathcal{U}^{\prime}} \alpha^{\prime}=\left.\int_{\mathcal{U}} \frac{d}{\mathrm{~d} t}\right|_{t=t_{0}}\left(\Xi_{t}^{*} \alpha^{\prime}\right)
$$

where $\mathcal{U}^{\prime}=\Xi_{t}(\mathcal{U})$. Thus

$$
\left.\frac{d}{\mathrm{~d} t}\right|_{t=t_{0}} \int_{\mathcal{U}^{\prime}} E^{\prime} \mathrm{d} V^{\prime}=\left.\int_{\mathcal{U}} \frac{d}{\mathrm{~d} t}\right|_{t=t_{0}}\left(\Xi_{t}^{*} E^{\prime}\right) \mathrm{d} V=\int_{\mathcal{U}}\left(\frac{\partial E}{\partial t}+\frac{\partial E}{\partial \mathbf{G}}: \mathfrak{L}_{\mathbf{W}} \mathbf{G}\right) \mathrm{d} V .
$$

Material balance of energy for $\mathcal{U}^{\prime} \subset \mathcal{B}^{\prime}$ at $t=t_{0}$ reads 


$$
\begin{aligned}
\int_{\mathcal{U}} & \frac{\partial \rho_{0}}{\partial t}\left(E+\frac{1}{2}\langle\langle\mathbf{V}-\mathbf{F W}, \mathbf{V}-\mathbf{F W}\rangle\rangle\right) \mathrm{d} V+\int_{\mathcal{U}} \rho_{0}\left(\frac{\partial E}{\partial t}+\frac{\partial E}{\partial \mathbf{G}}: \mathfrak{L}_{\mathbf{W}} \mathbf{G}+\left\langle\left\langle\mathbf{V}-\mathbf{F W},\left.\mathbf{A}^{\prime}\right|_{t=t_{0}}\right\rangle\right\rangle\right) \mathrm{d} V \\
= & \int_{\mathcal{U}} \rho_{0}\left(\left\langle\left\langle\left.\mathbf{B}^{\prime}\right|_{t=t_{0}}, \mathbf{V}-\mathbf{F W}\right\rangle\right\rangle+R\right) \mathrm{d} V+\int_{\partial \mathcal{U}}(\langle\langle\mathbf{T}, \mathbf{V}-\mathbf{F W}\rangle\rangle+H) \mathrm{d} A+\int_{\mathcal{U}}\left\langle\left\langle\mathbf{B}_{0}, \mathbf{W}\right\rangle\right\rangle \mathrm{d} V \\
& +\int_{\partial \mathcal{U}}\left\langle\left\langle\mathbf{T}_{0}, \mathbf{W}\right\rangle\right\rangle \mathrm{d} A .
\end{aligned}
$$

Note that $\mathbf{T}_{0}$ and $\mathbf{B}_{0}$ are defined on $\mathcal{B}$ and $\mathbf{T}_{0}^{\prime}$ and $\mathbf{B}_{0}^{\prime}$ are the corresponding quantities defined on $\Xi_{t}(\mathcal{B})$. Here we assume that $\mathbf{T}_{0}^{\prime}=\Xi_{t *} \mathbf{T}_{0}$ and $\mathbf{B}_{0}^{\prime}=\Xi_{t^{*}} \mathbf{B}_{0}$. Subtracting balance of energy for $\mathcal{U}$ from this and noting that $\left(\mathbf{A}^{\prime}-\mathbf{B}^{\prime}\right)_{t=t_{0}}=\mathbf{A}-\mathbf{B}$ we obtain

$$
\begin{aligned}
\int_{\mathcal{U}} \frac{\partial \rho_{0}}{\partial t}\left(-\langle\langle\mathbf{V}, \mathbf{F W}\rangle\rangle+\frac{1}{2}\langle\langle\mathbf{F W}, \mathbf{F W}\rangle) \mathrm{d} V+\int_{\mathcal{U}} \rho_{0}\left(\frac{\partial E}{\partial \mathbf{G}}: \mathfrak{L}_{\mathbf{W}} \mathbf{G}-\langle\langle\mathbf{F W}, \mathbf{A}\rangle\rangle\right) \mathrm{d} V\right. \\
=-\int_{\mathcal{U}}\left\langle\left\langle\rho_{0} \mathbf{B}, \mathbf{F W}\right\rangle\right\rangle \mathrm{d} V-\int_{\partial \mathcal{U}}\langle\langle\mathbf{T}, \mathbf{F W}\rangle\rangle \mathrm{d} A+\int_{\mathcal{U}}\left\langle\left\langle\mathbf{B}_{0}, \mathbf{W}\right\rangle\right\rangle \mathrm{d} V+\int_{\partial \mathcal{U}}\left\langle\left\langle\mathbf{T}_{0}, \mathbf{W}\right\rangle\right\rangle \mathrm{d} A .
\end{aligned}
$$

Cauchy's theorem implies that

$$
\langle\langle\mathbf{T}, \mathbf{F W}\rangle\rangle=\langle\langle\mathbf{F W},\langle\langle\mathbf{P}, \hat{\mathbf{N}}\rangle\rangle\rangle\rangle,
$$

where $\mathbf{P}$ is the first Piola-Kirchhoff stress tensor. Similarly

$$
\mathbf{T}_{0}=\left\langle\left\langle\mathbf{P}_{0}, \hat{\mathbf{N}}\right\rangle\right\rangle .
$$

Lemma 6.1: The surface integral in material energy balance has the following transformation:

$$
\int_{\partial \mathcal{U}}\left\langle\left\langle\mathbf{F}^{\top} \mathbf{T}, \mathbf{W}\right\rangle\right\rangle \mathrm{d} A=\int_{\mathcal{U}} \operatorname{Div}\left\langle\left\langle\mathbf{F}^{\top} \mathbf{P}, \mathbf{W}\right\rangle\right\rangle \mathrm{d} V=\int_{\mathcal{U}}\left[\left\langle\left\langle\operatorname{Div}\left(\mathbf{F}^{\top} \mathbf{P}\right), \mathbf{W}\right\rangle\right\rangle+\mathbf{F}^{\top} \mathbf{P}: \mathbf{\Omega}+\mathbf{F}^{\top} \mathbf{P}: \mathbf{K}\right] \mathrm{d} V,
$$

where

$$
\begin{gathered}
\mathbf{\Omega}_{I J}=\frac{1}{2}\left(G_{I K} W_{\mid J}^{K}-G_{J K} W_{\mid I}^{K}\right)=\frac{1}{2}\left(W_{I \mid J}-W_{J \mid I}\right), \\
\mathbf{K}_{I J}=\frac{1}{2}\left(G_{I K} W_{\mid J}^{K}+G_{J K} W_{\mid I}^{K}\right)=\frac{1}{2}\left(W_{I \mid J}+W_{J \mid I}\right), \quad \mathbf{K}=\frac{1}{2} \mathfrak{L}_{\mathbf{W}} \mathbf{G} .
\end{gathered}
$$

Proof: In components the integrand can be written as

$$
\left(\mathbf{F}^{\mathrm{\top}}\right)^{A}{ }_{a} T^{a} G_{A B} W^{B}
$$

But

$$
T^{a}=P^{a C} G_{C D} N^{D} .
$$

Hence in components the integrand reads

$$
\left(\left(\mathbf{F}^{\top}\right)^{A}{ }_{a} P^{a C} G_{A B} W^{B}\right) G_{C D} N^{D} .
$$

Using the divergence theorem the surface integral is transformed to an integral on $\mathcal{U}$ with the following integrand in components: 


$$
\begin{aligned}
\left(\left(\mathbf{F}^{\top}\right)^{A}{ }_{a} P^{a C} G_{A B} W^{B}\right)_{\mid C}= & \left(\left(\mathbf{F}^{\top} \mathbf{P}\right)^{A C} G_{A B} W^{B}\right)_{\mid C}=\left(\mathbf{F}^{\top} \mathbf{P}\right)^{A C}{ }_{\mid C} G_{A B} W^{B}+\left(\mathbf{F}^{\top} \mathbf{P}\right)^{A C} G_{A B} W^{B}{ }_{\mid C} \\
= & \left(\mathbf{F}^{\top} \mathbf{P}\right)^{A C}{ }_{\mid C} G_{A B} W^{B}+\left(\mathbf{F}^{\top} \mathbf{P}\right)^{A C}\left[\frac{1}{2}\left(G_{A B} W^{B}{ }_{\mid C}+G_{C B} W^{B}{ }_{\mid A}\right)+\frac{1}{2}\left(G_{A B} W^{B}{ }_{\mid C}\right.\right. \\
& \left.\left.-G_{C B} W^{B}{ }_{\mid A}\right)\right]
\end{aligned}
$$

where use was made of the fact that $G_{A B \mid C}=0$.

Similarly,

$$
\int_{\partial \mathcal{U}}\left\langle\left\langle\mathbf{T}_{0}, \mathbf{W}\right\rangle\right\rangle \mathrm{d} A=\int_{\mathcal{U}} \operatorname{Div}\left\langle\left\langle\mathbf{P}_{0}, \mathbf{W}\right\rangle\right\rangle \mathrm{d} V=\int_{\mathcal{U}}\left[\left\langle\left\langle\operatorname{Div}\left(\mathbf{P}_{0}\right), \mathbf{W}\right\rangle\right\rangle+\mathbf{P}_{0}: \mathbf{\Omega}+\mathbf{P}_{0}: \mathbf{K}\right] \mathrm{d} V
$$

By definition, at time $t=t_{0}$ the transformed balance of energy should be the same as the balance of energy for $\mathcal{U}$. Subtracting the material balance of energy for $\mathcal{U}$ from the above balance law and considering conservation of mass, we obtain

$$
\int_{\mathcal{U}} \rho_{0} \frac{\partial E}{\partial \mathbf{G}}: \mathfrak{L}_{\mathbf{W}} \mathbf{G} \mathrm{d} V+\int_{\mathcal{U}}\left\langle\left\langle\rho_{0} \mathbf{F}^{\top}(\mathbf{B}-\mathbf{A}), \mathbf{W}\right\rangle\right\rangle \mathrm{d} V-\int_{\mathcal{U}}\left\langle\left\langle\rho_{0} \mathbf{B}_{0}, \mathbf{W}\right\rangle\right\rangle \mathrm{d} V+\int_{\partial \mathcal{U}}\left\langle\left\langle\mathbf{F}^{\top} \mathbf{T}-\mathbf{T}_{0}, \mathbf{W}\right\rangle\right\rangle \mathrm{d} A=0 .
$$

Thus

$$
\begin{aligned}
& \int_{\mathcal{U}}\left(2 \rho_{0} \frac{\partial E}{\partial \mathbf{G}}+\mathbf{F}^{\top} \mathbf{P}-\mathbf{P}_{0}\right): \frac{1}{2} \mathfrak{L}_{\mathbf{W}} \mathbf{G} \mathrm{d} V+\int_{\mathcal{U}}\left(\mathbf{F}^{\top} \mathbf{P}-\mathbf{P}_{0}\right): \mathbf{\Omega} \mathrm{d} V+\int_{\mathcal{U}}\left\langle\left\langle\rho_{0} \mathbf{F}^{\top}(\mathbf{B}-\mathbf{A})-\mathbf{B}_{0}+\operatorname{Div}\left(\mathbf{F}^{\top} \mathbf{P}\right)\right.\right. \\
& \left.\left.-\operatorname{Div} \mathbf{P}_{0}, \mathbf{W}\right\rangle\right\rangle \mathrm{d} V=0 .
\end{aligned}
$$

Now using the balance of linear momentum the identity (6.43) simplifies to

$$
\begin{aligned}
& \int_{\mathcal{U}}\left(2 \rho_{0} \frac{\partial E}{\partial \mathbf{G}}+\mathbf{F}^{\top} \mathbf{P}-\mathbf{P}_{0}\right): \frac{1}{2} \mathfrak{L}_{\mathbf{W}} \mathbf{G} \mathrm{d} V+\int_{\mathcal{U}}\left(\mathbf{F}^{\top} \mathbf{P}-\mathbf{P}_{0}\right): \mathbf{\Omega} \mathrm{d} V+\int_{\mathcal{U}}\left\langle\left\langle\operatorname{Div}\left(\mathbf{F}^{\top} \mathbf{P}-\mathbf{P}_{0}\right)-\mathbf{F}^{\top} \operatorname{Div} \mathbf{P}\right.\right. \\
& \left.\left.\quad-\mathbf{B}_{0}, \mathbf{W}\right\rangle\right\rangle \mathrm{d} V=0 .
\end{aligned}
$$

Because $\mathcal{U}$ and $\mathbf{W}$ are arbitrary

$$
\begin{gathered}
\mathbf{P}_{0}=2 \rho_{0} \frac{\partial E}{\partial \mathbf{G}}+\mathbf{F}^{\top} \mathbf{P}, \\
\left(\mathbf{F}^{\top} \mathbf{P}-\mathbf{P}_{0}\right)^{\top}=\mathbf{F}^{\top} \mathbf{P}-\mathbf{P}_{0}, \\
\mathbf{B}_{0}=\operatorname{Div}\left(\mathbf{F}^{\top} \mathbf{P}-\mathbf{P}_{0}\right)-\mathbf{F}^{\top} \operatorname{Div} \mathbf{P} .
\end{gathered}
$$

Note that (6.46) is trivially satisfied after having (6.45). Thus we have

$$
\begin{gathered}
\mathbf{P}_{0}=2 \rho_{0} \frac{\partial E}{\partial \mathbf{G}}+\mathbf{F}^{\top} \mathbf{P}, \\
\mathbf{B}_{0}=\operatorname{Div}\left(\mathbf{F}^{\top} \mathbf{P}-\mathbf{P}_{0}\right)-\mathbf{F}^{\top} \operatorname{Div} \mathbf{P} .
\end{gathered}
$$

Note that $\mathbf{P}_{0}$ is a measure of anisotropy (deviation from material Doyle-Ericksen formula). This is an interesting result that in a natural way shows the contribution of some nonstandard terms to balance of energy when reference configuration evolves.

Thus we have proven the following theorem.

Theorem 6.2: Under a referential diffeomorphism $\Xi_{t}: \mathfrak{B} \rightarrow \mathfrak{B}$, and assuming that material energy density transforms tensorially, i.e., 


$$
E^{\prime}\left(\mathbf{X}^{\prime}, t, \mathbf{G}\right)=E\left(\mathbf{X}, t, \Xi_{t}^{*} \mathbf{G}\right),
$$

material energy balance has the following transformation:

$$
\begin{aligned}
\frac{d}{\mathrm{~d} t} \int_{\Xi_{t}(\mathcal{U})}\left(E^{\prime}+\frac{1}{2} \rho_{0}^{\prime}\left\langle\left\langle\mathbf{V}^{\prime}, \mathbf{V}^{\prime}\right\rangle\right\rangle\right) \mathrm{d} V^{\prime}= & \int_{\Xi_{t}(\mathcal{U})} \rho_{0}^{\prime}\left(\left\langle\left\langle\mathbf{B}^{\prime}, \mathbf{V}^{\prime}\right\rangle\right\rangle+R^{\prime}\right) \mathrm{d} V^{\prime}+\int_{\partial \Xi_{t}(\mathcal{U})}\left(\left\langle\left\langle\mathbf{T}^{\prime}, \mathbf{V}^{\prime}\right\rangle\right\rangle+H^{\prime}\right) \mathrm{d} A^{\prime} \\
& +\int_{\Xi_{t}(\mathcal{U})}\left\langle\left\langle\mathbf{B}_{0}^{\prime}, \mathbf{W}_{t}\right\rangle\right\rangle \mathrm{d} V^{\prime}+\int_{\partial \Xi_{t}(\mathcal{U})}\left\langle\left\langle\mathbf{T}_{0}^{\prime}, \mathbf{W}_{t}\right\rangle\right\rangle \mathrm{d} A^{\prime},
\end{aligned}
$$

where

$$
\begin{gathered}
\mathbf{T}_{0}^{\prime}=\Xi_{t^{*}}\left[\left\langle 2 \rho_{0} \frac{\partial E}{\partial \mathbf{G}}+\mathbf{F}^{\top} \mathbf{P}, \hat{\mathbf{N}} \|\right),\right. \\
\mathbf{B}_{0}^{\prime}=\Xi_{t^{*}}\left[\operatorname{Div}\left(\mathbf{F}^{\top} \mathbf{P}-\mathbf{P}_{0}\right)-\mathbf{F}^{\top} \operatorname{Div} \mathbf{P}\right],
\end{gathered}
$$

and the other quantities are already defined.

\section{B. Consequences of assuming invariance of energy balance}

This section shows the consequences of assuming material covariance of energy balance. It turns out that energy balance, in general, cannot be materially covariant.

Material energy balance is invariant under material diffeomorphisms if and only if the following relations hold between the nonstandard terms:

$$
\begin{gathered}
\mathbf{P}_{0}=\mathbf{0} \quad \text { or } \quad 2 \rho_{0} \frac{\partial E}{\partial \mathbf{G}}=-\mathbf{F}^{\top} \mathbf{P}, \\
\mathbf{B}_{0}=\mathbf{0} \quad \text { or } \quad \operatorname{Div}\left(\mathbf{F}^{\top} \mathbf{P}\right)=\mathbf{F}^{\top} \operatorname{Div} \mathbf{P} .
\end{gathered}
$$

Equation (6.54) is the material Doyle-Ericksen formula and (6.55) is the configurational inviscidity constraint, which will be defined in the sequel. Let us now start with the "naive" assumption that energy balance is materially covariant and see what its consequences are.

Material covariance of energy balance: Let us postulate that the balance of energy is invariant under a diffeomorphism $\Xi_{t}: \mathfrak{B} \rightarrow \mathfrak{B}$, i.e.,

$$
\frac{d}{\mathrm{~d} t} \int_{\mathcal{U}^{\prime}} \rho_{0}^{\prime}\left(E^{\prime}+\frac{1}{2}\left\langle\left\langle\mathbf{V}^{\prime}, \mathbf{V}^{\prime}\right\rangle\right\rangle\right) \mathrm{d} V^{\prime}=\int_{\mathcal{U}^{\prime}} \rho_{0}^{\prime}\left(\left\langle\left\langle\mathbf{B}^{\prime}, \mathbf{V}^{\prime}\right\rangle\right\rangle+R^{\prime}\right) \mathrm{d} V^{\prime}+\int_{\partial \mathcal{U}^{\prime}}\left(\left\langle\left\langle\mathbf{T}^{\prime}, \mathbf{V}^{\prime}\right\rangle\right\rangle+H^{\prime}\right) \mathrm{d} A^{\prime} .
$$

Proposition 6.3: If material energy balance is invariant under arbitrary material diffeomorphisms $\Xi_{t}: \mathfrak{B} \rightarrow \mathfrak{B}$, then

$$
\begin{gathered}
\frac{\partial \rho_{0}}{\partial t}=0, \\
2 \rho_{0} \frac{\partial E}{\partial \mathbf{G}}=-\mathbf{F}^{\top} \mathbf{P}, \\
\mathbf{F}^{\top} \mathbf{P}=\mathbf{P}^{\top} \mathbf{F}, \\
\operatorname{Div}\left(\mathbf{F}^{\top} \mathbf{P}\right)=\mathbf{F}^{\top} \operatorname{Div} \mathbf{P} .
\end{gathered}
$$


Conversely, if the above four conditions hold, then material energy balance is invariant under any material diffeomorphism.

Proof: Material balance of energy for $\mathcal{U}^{\prime} \subset \mathcal{B}^{\prime}$ at $t=t_{0}$ reads

$$
\begin{gathered}
\int_{\mathcal{U}} \frac{\partial \rho_{0}}{\partial t}\left(E+\frac{1}{2}\langle\langle\mathbf{V}-\mathbf{F W}, \mathbf{V}-\mathbf{F W}\rangle\rangle\right) \mathrm{d} V+\int_{\mathcal{U}} \rho_{0}\left(\frac{\partial E}{\partial t}+\frac{\partial E}{\partial \mathbf{G}}: \mathfrak{L}_{\mathbf{W}} \mathbf{G}+\left\langle\left\langle\mathbf{V}-\mathbf{F W},\left.\mathbf{A}^{\prime}\right|_{t=t_{0}}\right\rangle\right\rangle \mathrm{d} V\right. \\
=\int_{\mathcal{U}} \rho_{0}\left(\left\langle\left\langle\left.\mathbf{B}^{\prime}\right|_{t=t_{0}}, \mathbf{V}-\mathbf{F W}\right\rangle\right\rangle+R\right) \mathrm{d} V+\int_{\partial \mathcal{U}}(\langle\langle\mathbf{T}, \mathbf{V}-\mathbf{F W}\rangle\rangle+H) \mathrm{d} A .
\end{gathered}
$$

Subtracting balance of energy for $\mathcal{U} \subset \mathcal{B}$ from this and noting that $\left(\mathbf{A}^{\prime}-\mathbf{B}^{\prime}\right)_{t=t_{0}}=\mathbf{A}-\mathbf{B}$ we obtain

$$
\begin{aligned}
\int_{\mathcal{U}} & \frac{\partial \rho_{0}}{\partial t}\left(-\langle\langle\mathbf{V}, \mathbf{F W}\rangle\rangle+\frac{1}{2}\langle\langle\mathbf{F W}, \mathbf{F W}\rangle\rangle\right) \mathrm{d} V+\int_{\mathcal{U}} \rho_{0}\left(\frac{\partial E}{\partial \mathbf{G}}: \mathfrak{L}_{\mathbf{W}} \mathbf{G}-\langle\langle\mathbf{F W}, \mathbf{A}\rangle\rangle\right) \mathrm{d} V \\
= & -\int_{\mathcal{U}}\left\langle\left\langle\rho_{0} \mathbf{B}, \mathbf{F W}\right\rangle\right\rangle \mathrm{d} V-\int_{\partial \mathcal{U}}\langle\langle\mathbf{T}, \mathbf{F W}\rangle\rangle \mathrm{d} A .
\end{aligned}
$$

We know that

$$
\int_{\partial \mathcal{U}}\left\langle\left\langle\mathbf{F}^{\top} \mathbf{T}, \mathbf{W}\right\rangle\right\rangle \mathrm{d} A=\int_{\mathcal{U}}\left[\left\langle\left\langle\operatorname{Div}\left(\mathbf{F}^{\top} \mathbf{P}\right), \mathbf{W}\right\rangle\right\rangle+\mathbf{F}^{\top} \mathbf{P}: \mathbf{\Omega}+\mathbf{F}^{\top} \mathbf{P}: \frac{1}{2} \mathfrak{L}_{\mathbf{W}} \mathbf{G}\right] \mathrm{d} V .
$$

Thus, (6.62) simplifies to

$$
\begin{aligned}
& \int_{\mathcal{U}} \frac{\partial \rho_{0}}{\partial t}\left(-\langle\langle\mathbf{V}, \mathbf{F W}\rangle\rangle+\frac{1}{2}\langle\langle\mathbf{F W}, \mathbf{F W}\rangle\rangle\right) \mathrm{d} V+\int_{\mathcal{U}}\left(2 \rho_{0} \frac{\partial E}{\partial \mathbf{G}}+\mathbf{F}^{\top} \mathbf{P}\right): \frac{1}{2} \mathfrak{L}_{\mathbf{W}} \mathbf{G} \mathrm{d} V+\int_{\mathcal{U}} \mathbf{F}^{\top} \mathbf{P}: \mathbf{\Omega} \mathrm{d} V \\
& \quad+\int_{\mathcal{\partial U}}\left\langle\left\langle\operatorname{Div}\left(\mathbf{F}^{\top} \mathbf{P}\right)+\rho_{0} \mathbf{F}^{\top}(\mathbf{B}-\mathbf{A}), \mathbf{W}\right\rangle\right\rangle \mathrm{d} V=0
\end{aligned}
$$

As $\mathcal{U}$ and $\mathbf{W}$ are arbitrary, we have

$$
\begin{gathered}
\frac{\partial \rho_{0}}{\partial t}=0, \\
2 \rho_{0} \frac{\partial E}{\partial \mathbf{G}}=-\mathbf{F}^{\top} \mathbf{P}, \\
\mathbf{F}^{\top} \mathbf{P}=\mathbf{P}^{\top} \mathbf{F}, \\
\operatorname{Div}\left(\mathbf{F}^{\top} \mathbf{P}\right)+\rho_{0} \mathbf{F}^{\top} \mathbf{B}=\rho_{0} \mathbf{F}^{\top} \mathbf{A} .
\end{gathered}
$$

Equation (6.65) is nothing but material conservation of mass. Equation (6.66) is the material Doyle-Ericksen formula. This is what $\mathrm{Lu}$ and Papadopoulos ${ }^{26}$ call infinitesimal material covariance. Equation (6.67) is balance of configurational angular momentum or isotropy of the material. [Note that if (6.66) holds then (6.67) holds trivially.] Finally, Eq. (6.68) is a condition that must be satisfied for the balance of energy to be invariant under material diffeomorphisms. This constraint is equivalent to

$$
\operatorname{Div}\left(\mathbf{F}^{\top} \mathbf{P}\right)=\mathbf{F}^{\top} \operatorname{Div} \mathbf{P}
$$

Assuming the above four conditions, it is easy to show that material energy balance is invariant under arbitrary material diffeomorphisms. 
Ideal fluids do satisfy all these conditions. In fact, their transformation properties under material diffeomorphisms gives rise to Kelvin's circulation theorem and it is a key ingredient in the geometric approach to fluid mechanics; see the introduction to the Marsden and Ratiu book ${ }^{29}$ for a discussion and references to the literature.

\section{Material energy balance and defects}

We now make a connection between (6.69) and Eshelby's idea of force on a defect. The idea of a driving force in continuum mechanics goes back to Eshelby ${ }^{13-15}$ and this notion is important in developing evolution laws for the movement of defects, including dislocations, vacancies, interfaces, cavities, cracks, etc. Driving forces on these defects cause climb and glide of dislocations, diffusion of point defects, migration of interfaces, changing the shape of cavities and propagation of cracks, to mention a few examples. Eshelby defined the force on a defect as the generalized force corresponding to position of the defect (in the reference configuration), which is thought of as a generalized displacement. Eshelby studied inhomogeneities in elastostatic and elastodynamic systems by considering the explicit dependence of the elastic energy density on position in the reference configuration.

Defect forces: Suppose the elastic energy density has an explicit dependence on $\mathbf{X}$ (the position of material points in the undeformed configuration), i.e.,

$$
W=W(\varphi, \mathbf{F}, \mathbf{X}),
$$

where $\varphi$ and $\mathbf{F}$ are the deformation mapping and the deformation gradient, respectively. Consider an open neighborhood $\Omega$ of an isolated defect. Force on the defect in the sense of Eshelby is defined as

$$
\mathbf{F}^{\text {defect }}=\int_{\Omega}\left(\frac{\partial W}{\partial \mathbf{X}}\right)_{\text {explicit }} \mathrm{d} V=\int_{\Omega} \operatorname{Div} \mathbf{E} \mathrm{d} V=\int_{\partial \Omega} \mathbf{E} \hat{\mathbf{N}} \mathrm{d} A,
$$

where $\mathbf{E}=W \mathbf{I}-\mathbf{F}^{\top} \mathbf{P}$ is Eshelby's energy-momentum tensor. It turns out that for a crack (thought of as a defect) $\mathbf{F}^{\text {defect }}$ is related to the celebrated $J$-integral; ${ }^{37} J$ is the component of $\mathbf{F}^{\text {defect }}$ in the direction of crack propagation.

The following proposition makes an explicit connection between (6.69) and Eshelby's idea of force on a defect.

Proposition 6.4: Suppose an elastic material in an isothermal and quasistatic deformation satisfies the internal constraint $\operatorname{Div}\left(\mathbf{F}^{\top} \mathbf{P}\right)=\mathbf{F}^{\top}$ Div $\mathbf{P}$. In the absence of body forces, force on a defect in the sense of Eshelby would be

$$
\mathbf{F}^{\text {defect }}=\int_{\Omega}\left(\frac{\partial W}{\partial \mathbf{X}}\right)_{\text {explicit }} \mathrm{d} V=\int_{\Omega} \operatorname{Div} \mathbf{E} \mathrm{d} V=\int_{\partial \Omega} W \hat{\mathbf{N}} \mathrm{d} A .
$$

Proof: Note that

$$
\mathbf{F}^{\text {defect }}=\int_{\partial \Omega} \mathbf{E} \hat{\mathbf{N}} \mathrm{d} A=\int_{\partial \Omega} W \hat{\mathbf{N}} \mathrm{d} A-\int_{\Omega} \mathbf{F}^{\top} \operatorname{Div} \mathbf{P} \mathrm{d} V=\int_{\partial \Omega} W \hat{\mathbf{N}} \mathrm{d} A .
$$

This means that the configurational traction on $\partial \Omega$ is normal to $\partial \Omega$ at all points, i.e., the configurational stress is hydrostatic. For this reason we call the internal constraint $\operatorname{Div}\left(\mathbf{F}^{\top} \mathbf{P}\right)=\mathbf{F}^{\top} \operatorname{Div} \mathbf{P}$, the configurational inviscidity constraint.

If there is a stationary surface $\mathfrak{S}$ across which deformation gradient and other quantities have jump discontinuities, the balance of standard forces reads

$$
\llbracket \mathbf{P} \rrbracket \hat{\mathbf{N}}=\mathbf{0} .
$$

Now let us look at the normal jump in Eshelby's energy-momentum tensor, 


$$
\llbracket \mathbf{E} \rrbracket \hat{\mathbf{N}}=\llbracket \Psi \mathbf{I}-\mathbf{F}^{\top} \mathbf{P} \rrbracket \hat{\mathbf{N}}=\llbracket \Psi \rrbracket \hat{\mathbf{N}}-\llbracket \mathbf{F}^{\top} \rrbracket\langle\mathbf{P}\rangle \hat{\mathbf{N}}-\left\langle\mathbf{F}^{\top}\right\rangle \llbracket \mathbf{P} \rrbracket \hat{\mathbf{N}},
$$

where $\langle\cdot\rangle$ denotes average of inner and outer traces. Using Hadamard's compatibility equations,

$$
\llbracket \mathbf{F} \rrbracket \hat{\mathbf{t}}=\mathbf{0} \text { for all } \hat{\mathbf{t}} \text { such that } \hat{\mathbf{t}} \cdot \hat{\mathbf{N}}=0,
$$

it can be easily shown that

$$
\hat{\mathbf{N}} \cdot \llbracket \mathbf{E} \rrbracket \hat{\mathbf{N}}=\llbracket \Psi \rrbracket-\llbracket \mathbf{F} \rrbracket \hat{\mathbf{N}} \cdot \mathbf{P} \hat{\mathbf{N}}, \quad \hat{\mathbf{t}} \cdot \llbracket \mathbf{E} \rrbracket \hat{\mathbf{N}}=-\mathbf{F} \hat{\mathbf{t}} \cdot \llbracket \mathbf{P} \rrbracket \hat{\mathbf{N}} .
$$

Now if the balance of standard forces hold one concludes that

$$
\hat{\mathbf{t}} \cdot \llbracket \mathbf{E} \rrbracket \hat{\mathbf{N}}=0 .
$$

This means that jump in configurational traction on $\partial \Omega$ is always normal to $\partial \Omega$. However, the previous remark shows that in the absence of body forces the condition $\operatorname{Div}\left(\mathbf{F}^{\top} \mathbf{P}\right)=\mathbf{F}^{\top} \operatorname{Div} \mathbf{P}$ implies that the configurational traction itself is normal to $\partial \Omega$.

Are configurational forces newtonian?: There have been doubts and discussions concerning the nature of configurational forces in the literature already starting from Eshelby himself. Eshelby strongly believed that force on a defect is fictitious and is different from the usual forces in mechanics. He defined force on a defect to be the thermodynamic force conjugate to the generalized coordinates defining the defect, for example, the crack tip position in the case of a crack. Eshelby ${ }^{16}$ observed that the configurational force on a disclination in a liquid crystal is a real force. A similar observation was made by $\mathrm{Nabarro}^{34}$ for dislocations. Kröner ${ }^{23}$ and Ericksen ${ }^{11,12}$ have similar discussions. Batra ${ }^{4}$ argues that force on a defect is equal to the standard force exerted on the boundary of a subbody embracing the defect. Steinmann ${ }^{42}$ introduces the spatial signature of a material force. One should note that this viewpoint is not in agreement with Gurtin's theory in which standard and configurational forces have their own balance laws.

Batra ${ }^{4}$ proves a theorem that states that force on a defect is equal to the resultant of tractions on the boundary of any region enclosing this single defect. This seems to be a very surprising result. First of all, if body forces are considered resultant of tractions on different regions embodying the defect cannot be independent of the region as in this case stress tensor is not divergence free. Barta suggests that problems involving the $J$-integral could be reinterpreted using his theorem. As a matter of fact, the $J$-integral can serve as a counter example for Batra's theorem. The reason is that in the case of a linear elastic material in mode I fracture, for example, the $J$-integral is quadratic in $K_{I}$ while the stress is linear in $K_{I}$ and hence the resultant of tractions acting on the boundary of a small region enclosing the crack would be linear in $K_{I}$. This means that the $J$-integral, which is the component of configurational force in the direction of crack growth, cannot be a real force. The incorrectness of Batra's theorem is because of the way he defines force on a defect. Force on a defect in the sense of Eshelby is the rate of change of potential energy of the elastic body with respect to changes in the position of the defect in the reference configuration. Batra defines force on a defect to be the rate of change of energy with respect to changes of position of the defect in the current configuration. This is the source of his surprising result. One should note that direct and inverse motions describe the same physical process and cannot lead to different conclusions regarding forces. Having the duality picture is useful but one should note that positions of defects in the reference and current configurations are not related by the standard deformation mapping as the evolution of defects is an independent kinematical process.

Standard forces in continuum mechanics are one forms in the deformed configuration, i.e., at each point $x \in \mathcal{S}$, force is an element of $T_{x}^{*} \mathcal{S}$. Configurational forces on the other hand are one forms in the reference configuration, i.e., at each point $X \in \mathcal{B}$, configurational force is an element of $T_{X}^{*} \mathcal{B}$. Therefore, geometrically it is meaningless to ask if a configurational force is a real force 
very much like asking whether the deformation gradient (a two-point tensor) is symmetric. This is why arguments like the one proposed by Steinmann ${ }^{43}$ where he defines a spatial signature for a material force do not make sense from the geometric standpoint.

Plasticity and embeddings: A traditional means of introducing configurational forces is based on remapping the reference configuration of the body. However, this approach tacitly assumes that the reference configuration can be embedded in Euclidean space. This approach fails when there is no natural embedding of the reference configuration. A case in point is provided by multiplicative plasticity, ${ }^{24}$ where the total deformation gradient at a point $x$ has the representation: $\mathbf{F}(x)$ $=\mathbf{F}^{e}(x) \mathbf{F}^{p}(x)$, where $\mathbf{F}^{e}(x)$ and $\mathbf{F}^{p}(x)$ are the elastic and plastic deformation gradients, both of which fail to be a gradient in general. The plastic deformation mapping $\mathbf{F}^{p}(x)$ defines an intermediate configuration that defines the reference configuration for the elasticity of the material. In particular, the elastic energy density is assumed to be of the form $W\left(\mathbf{F}^{e}, x\right)$. Since $\mathbf{F}^{p}(x)$ is not the derivative of a mapping, the intermediate configuration cannot be embedded in Euclidean space. Therefore, remapping cannot be applied to deriving configurational forces directly from $W\left(\mathbf{F}^{e}, x\right)$. By contrast, the present approach can be applied for that purpose, for example, by equipping the intermediate configuration with a constant metric.

The derivation of certain conserved integrals, such as the $L$-integral that gives the configurational torque on isotropic subbodies, relies on the metric structure of the embedding Euclidean space. In addition, the conventional formulation of material symmetry also presumes the existence of an Euclidian embedding. Such an embedding may not be natural or available in certain models of materials, such as liquid crystals or smectic polymers, where the reference configuration may include a unit director field.

\section{NOETHER'S THEOREM AND BALANCE OF CONFIGURATIONAL FORCES}

As is well known, there is a strong connection between conservation laws and symmetries. If the Euler-Lagrange equations are satisfied and the Lagrangian density of a system is invariant under a group of transformations, Noether's theorem gives the corresponding conserved quantity. In this sense, conservation laws are related to symmetries of a given system. Marsden and Hughes $^{28}$ consider material invariance in elasticity (in the absence of body forces) and show that invariance of Lagrangian density under rigid translations in the reference configuration results in the following conservation law

$$
\frac{\partial}{\partial t}\left(\partial_{\dot{\phi}} \mathfrak{L} \cdot D \phi \cdot \mathbf{W}\right)+\operatorname{DIV}\left(\partial_{\mathbf{F}} \mathfrak{L} \cdot D \phi \cdot \mathbf{W}-\mathfrak{L} \mathbf{W}\right)=0 .
$$

This has been obtained assuming that the flow of $\mathbf{W}$ is volume-preserving and that $\mathcal{L}$ does not explicitly depend on $X$. For a constant $\mathbf{W}$, this equation in our notation reads

$$
\operatorname{Div}\left[\left(\Psi-\frac{1}{2} \rho_{0}|\mathbf{V}|^{2}\right) \mathbf{I}-\mathbf{F}^{\top} \mathbf{P}\right]=-\frac{\partial}{\partial t}\left(\rho_{0} \mathbf{F}^{\top} \mathbf{V}\right) .
$$

It is seen that this is identical to balance of configurational linear momentum if $\rho_{0}$ and $\Psi$ are independent of $\mathbf{X}$ (note that this is stronger that homogeneity of $\mathfrak{L}$ ). Ignoring the inertial effects, Noether's theorem results in

$$
\operatorname{Div}\left(\Psi \mathbf{I}-\mathbf{F}^{\top} \mathbf{P}\right)=\mathbf{0} .
$$

Roughly speaking, Noether's theorem states that when the Euler-Lagrange equations are satisfied, any symmetry of the Lagrangian density corresponds to a conserved quantity. Here we revisit Noether's theorem for nonlinear elasticity assuming that undeformed and deformed configurations are Riemannian manifolds. Writing action in the reference configuration, Lagrangian density has the following explicit independent variables: 


$$
\mathcal{L}=\mathcal{L}\left(X^{A}, \varphi^{a}, \dot{\varphi}^{a}, F^{a}{ }_{A}, G_{A B}, g_{a b}\right) .
$$

For the sake of clarity, we consider spatial and material symmetries of the Lagrangian density separately.

\section{A. Spatial covariance of Lagrangian density}

Theorem 7.1: If the Lagrangian density is spatially covariant, then the following hold: (i) spatial homogeneity of the Lagrangian density and (ii) the Doyle-Ericksen formula.

Proof: Suppose $\psi_{s}$ is a flow on $\mathcal{S}$ generated by a vector field $\mathbf{w}$, i.e.,

$$
\left.\frac{d}{\mathrm{~d} s}\right|_{s=0} \psi_{s} \circ \varphi=\mathbf{w} \circ \varphi
$$

Invariance of $\mathcal{L}$ means that

$$
\mathcal{L}\left(X^{A}, \psi_{s}^{a}(\varphi), \frac{\partial \psi_{s}^{a}}{\partial x^{b}} \dot{\varphi}^{b}, \frac{\partial \psi_{s}^{a}}{\partial x^{b}} F^{b}{ }_{A}, G_{A B},-\frac{\partial \psi_{s}^{c}}{\partial x^{a}} \frac{\partial \psi_{s}^{d}}{\partial x^{b}} g_{c d}\right)=\mathcal{L}\left(X^{A}, \varphi^{a}, \dot{\varphi}^{a}, F^{a}{ }_{A}, G_{A B}, g_{a b}\right)
$$

[This reminds us of the definition of covariance for internal energy density. So, it would be very natural to expect some connection between Noether's theorem and covariant balance laws.] Now differentiating the above relation with respect to $s$ and then evaluating it at $s=0$ [This is somewhat similar to subtracting two balance relations and evaluating the result at $\left.t=t_{0}\right]$, one obtains

$$
\frac{\partial \mathcal{L}}{\partial \varphi^{a}} w^{a}+\frac{\partial \mathcal{L}}{\partial \dot{\varphi}^{a}} \frac{\partial w^{a}}{\partial x^{b}} \dot{\varphi}^{b}+\left(\frac{\partial \mathcal{L}}{\partial \mathbf{F}}\right)_{a}^{A} \frac{\partial w^{a}}{\partial x^{b}} F_{A}^{b}-2 \frac{\partial \mathcal{L}}{\partial g_{a b}} \frac{\partial w^{c}}{\partial x^{a}} g_{b c}=0 .
$$

Note that

$$
\frac{\partial \mathcal{L}}{\partial \dot{\varphi}^{a}} \frac{\partial w^{a}}{\partial x^{b}} \dot{\varphi}^{b}=\frac{\partial}{\partial t}\left(\frac{\partial \mathcal{L}}{\partial \dot{\varphi}^{a}} w^{a}\right)-\frac{\partial}{\partial t}\left(\frac{\partial \mathcal{L}}{\partial \dot{\varphi}^{a}}\right) w^{a} .
$$

After some manipulations, it can be shown that

$$
\left(\frac{\partial \mathcal{L}}{\partial \mathbf{F}}\right)_{a}^{A} \frac{\partial w^{a}}{\partial x^{b}} F_{A}^{b}=\left[\left(\frac{\partial \mathcal{L}}{\partial \mathbf{F}}\right)_{a}^{A} w^{a}\right]_{\mid A}-\left(\frac{\partial \mathcal{L}}{\partial \mathbf{F}}\right)_{a}^{A} w^{a}-\left(\frac{\partial \mathcal{L}}{\partial \mathbf{F}}\right)_{b}^{A} \gamma_{a c}^{b} F_{A}^{c} w^{a}
$$

Also

$$
-2 \frac{\partial \mathcal{L}}{\partial g_{a b}} \frac{\partial w^{c}}{\partial x^{a}} g_{b c}=-\left[2 \frac{\partial \mathcal{L}}{\partial g_{a b}} g_{b c}\left(\mathbf{F}^{-1}\right)_{a}^{A} w^{c}\right]_{\mid A}+\left[2 \frac{\partial \mathcal{L}}{\partial g_{a b}} g_{b c}\left(\mathbf{F}^{-1}\right)_{a}^{A}\right]_{\mid A}^{w^{c}}+2 \frac{\partial \mathcal{L}}{\partial g_{a b}} g_{b d} \gamma_{a c}^{d} w^{c}
$$

Therefore, symmetry of $\mathcal{L}$ implies that

$$
\begin{gathered}
{\left[\frac{\partial \mathcal{L}}{\partial \varphi^{a}}-\frac{\partial}{\partial t}\left(\frac{\partial \mathcal{L}}{\partial \dot{\varphi}}\right)_{a}-\left(\frac{\partial \mathcal{L}}{\partial \mathbf{F}}\right)_{a}^{A}-\left(\frac{\partial \mathcal{L}}{\partial \mathbf{F}}\right)_{b}^{A} F_{A}^{c} \gamma_{a c}^{b}+2 \frac{\partial \mathcal{L}}{\partial g_{c d}} g_{b d} \gamma_{a c}^{b}\right]^{a}+\frac{\partial}{\partial t}\left(\frac{\partial \mathcal{L}}{\partial \dot{\varphi}^{a}} w^{a}\right)} \\
+\left[\left(\frac{\partial \mathcal{L}}{\partial \mathbf{F}}\right)_{a}^{A} w^{a}\right]_{\mid A}-\left[2 \frac{\partial \mathcal{L}}{\partial g_{a b}} g_{b c}\left(\mathbf{F}^{-1}\right)_{a}^{A} w^{c}\right]_{\mid A}+\left[2 \frac{\partial \mathcal{L}}{\partial g_{a b}} g_{b c}\left(\mathbf{F}^{-1}\right)_{a}{ }^{A}\right]_{\mid A} w^{c}=0 .
\end{gathered}
$$

Note that the term multiplied by $w^{a}$ is zero if the Euler-Lagrange equations are satisfied. Thus, Noether's theorem states that 


$$
\frac{\partial}{\partial t}\left(\frac{\partial \mathcal{L}}{\partial \dot{\varphi}^{a}} w^{a}\right)+\left[\left(\frac{\partial \mathcal{L}}{\partial \mathbf{F}}\right)_{a}^{A} w^{a}\right]_{\mid A}-\left[2 \frac{\partial \mathcal{L}}{\partial g_{a b}} g_{b c}\left(\mathbf{F}^{-1}\right)_{a}{ }^{A} w^{c}\right]_{\mid A}+\left[2 \frac{\partial \mathcal{L}}{\partial g_{a b}} g_{b c}\left(\mathbf{F}^{-1}\right)_{a}{ }^{A}\right]_{\mid A} w^{c}=0
$$

Note that

$$
\left[\left(\frac{\partial \mathcal{L}}{\partial \mathbf{F}}\right)_{a}^{A} w^{a}\right]_{\mid A}=\left(\frac{\partial \mathcal{L}}{\partial \mathbf{F}}\right)_{a \mid A}^{A} w^{a}+\left(\frac{\partial \mathcal{L}}{\partial \mathbf{F}}\right)_{a}^{A} F_{A}^{b} w^{a}{ }_{\mid b}
$$

Also

$$
\left[2 \frac{\partial \mathcal{L}}{\partial g_{a b}} g_{b c}\left(\mathbf{F}^{-1}\right)_{a}{ }^{A} w^{c}\right]_{\mid A}=\left[2 \frac{\partial \mathcal{L}}{\partial g_{a b}} g_{b c}\left(\mathbf{F}^{-1}\right)_{a}{ }^{A}\right]_{\mid A} w^{c}+2 \frac{\partial \mathcal{L}}{\partial g_{a b}} g_{b c} w^{c}{ }_{\mid a} .
$$

Therefore (7.12) is simplified to

$$
\left[\frac{\partial}{\partial t}\left(\frac{\partial \mathcal{L}}{\partial \dot{\varphi}^{a}}\right)+\left(\frac{\partial \mathcal{L}}{\partial \mathbf{F}}\right)_{a \mid A}^{A}\right] w^{a}+\left(\frac{\partial \mathcal{L}}{\partial \dot{\varphi}^{a}}\right) \frac{\partial w^{a}}{\partial t}+\left[\left(\frac{\partial \mathcal{L}}{\partial \mathbf{F}}\right)_{a}^{A} F_{A}^{c}-2 \frac{\partial \mathcal{L}}{\partial g_{b c}} g_{a b}\right] w^{a}{ }_{\mid c}=0 .
$$

Note that

$$
\frac{\partial w^{a}}{\partial t}=\frac{\partial w^{a}}{\partial x^{c}} \dot{\varphi}^{c}=\dot{\varphi}^{c} w^{a}{ }_{c}-\gamma_{c d^{a}}^{a} w^{d} \dot{\varphi}^{c}
$$

Therefore statement of Noether's theorem, Eq. (7.12) can be rewritten as

$$
\left[\frac{\partial}{\partial t}\left(\frac{\partial \mathcal{L}}{\partial \dot{\varphi}^{a}}\right)+\left(\frac{\partial \mathcal{L}}{\partial \mathbf{F}}\right)_{a \mid A}^{A}-\frac{\partial \mathcal{L}}{\partial \dot{\varphi}^{d}} \gamma_{a c}^{d} \dot{\varphi}^{c}\right] w^{a}+\left[\left(\frac{\partial \mathcal{L}}{\partial \mathbf{F}}\right)_{a}^{A} F^{c}{ }_{A}+\frac{\partial \mathcal{L}}{\partial \dot{\varphi}^{a}} \dot{\varphi}^{c}-2 \frac{\partial \mathcal{L}}{\partial g_{b c}} g_{a b}\right] w^{a}{ }_{c}=0
$$

Note that

$$
\frac{\partial \mathcal{L}}{\partial \dot{\varphi}^{a}} \dot{\varphi}^{c}=\rho_{0} g_{a b} \dot{\varphi}^{b} \dot{\varphi}^{c}
$$

If Lagrangian density is covariant, i.e., if $\mathbf{w}$ is arbitrary then (7.17) implies that

$$
\begin{gathered}
2 \frac{\partial \mathcal{L}}{\partial g_{a b}}=g^{b c}\left(\frac{\partial \mathcal{L}}{\partial \mathbf{F}}\right)_{c}^{A} F_{A}^{a}+g^{b c} \frac{\partial \mathcal{L}}{\partial \dot{\varphi}^{c}} \dot{\varphi}^{a}, \\
\frac{\partial}{\partial t}\left(\frac{\partial \mathcal{L}}{\partial \dot{\varphi}^{a}}\right)+\left(\frac{\partial \mathcal{L}}{\partial \mathbf{F}}\right)_{a \mid A}^{A}-\frac{\partial \mathcal{L}}{\partial \dot{\varphi}^{d}} \gamma_{a c}^{d} \dot{\varphi}^{c}=0 .
\end{gathered}
$$

Equation (7.19) can be rewritten as

$$
2 \frac{\partial W}{\partial g_{a b}}=g^{b c}\left(\frac{\partial W}{\partial \mathbf{F}}\right)_{c}^{A} F_{A}^{a},
$$

which is nothing but the Doyle-Ericksen formula. [Note that this includes balance of angular momentum.] Note that 


$$
\frac{\partial}{\partial t}\left(\frac{\partial \mathcal{L}}{\partial \dot{\varphi}^{a}}\right)+\left(\frac{\partial \mathcal{L}}{\partial \mathbf{F}}\right)_{a \mid A}^{A}-\frac{\partial \mathcal{L}}{\partial \dot{\varphi}^{d}} \gamma_{a c}^{d} \dot{\varphi}^{c}=\frac{\partial \mathcal{L}}{\partial \varphi^{a}}-\left(\frac{\partial \mathcal{L}}{\partial \mathbf{F}}\right)_{b}^{A} F_{A}^{c} \gamma_{a c}^{b}+2 \frac{\partial \mathcal{L}}{\partial g_{c d}} g_{b d} \gamma_{a c}^{b}-\frac{\partial \mathcal{L}}{\partial \dot{\varphi}^{b}} \dot{\varphi}^{c} \gamma_{a c}^{b}
$$

But

$$
-\left(\frac{\partial \mathcal{L}}{\partial \mathbf{F}}\right)_{b}^{A} F_{A}^{c}+2 \frac{\partial \mathcal{L}}{\partial g_{c d}} g_{b d}=\frac{\partial \mathcal{L}}{\partial \dot{\varphi}^{b}} \dot{\varphi}^{c}
$$

Thus

$$
\frac{\partial}{\partial t}\left(\frac{\partial \mathcal{L}}{\partial \dot{\varphi}^{a}}\right)+\left(\frac{\partial \mathcal{L}}{\partial \mathbf{F}}\right)_{a \mid A}^{A}-\frac{\partial \mathcal{L}}{\partial \dot{\varphi}^{d}} \gamma_{a c}^{d} \dot{\varphi}^{c}=\frac{\partial \mathcal{L}}{\partial \varphi^{a}}
$$

Hence (7.20) implies that

$$
\frac{\partial \mathcal{L}}{\partial \varphi^{a}}=0
$$

Note that this theorem implies that arbitrary flows and in particular rigid translations cannot be transitive (in the sense of Gotay et al. ${ }^{17,18}$ ) for arbitrary Lagrangian densities.

\section{B. Material covariance of Lagrangian density}

Let us first consider the case of Euclidean spaces. Consider a flow $\Lambda_{s}$ on $\mathcal{B}$ generated by a vector field $\mathbf{W}$. Invariance of $\mathcal{L}$ with respect to this flow means that

$$
\mathcal{L}\left(\Lambda_{s}^{A}(X), \varphi^{a}, \dot{\varphi}^{a},\left[\left(\frac{\partial \Lambda_{s}}{\partial X}\right)^{-1}\right]_{A}^{B} F_{B}^{a}\right)=\mathcal{L}\left(X^{A}, \varphi^{a}, \dot{\varphi}^{a}, F^{a}{ }_{A}\right) .
$$

Differentiating the above relation with respect to $s$ and evaluating the result at $s=0$, one obtains

$$
\frac{\partial \mathcal{L}}{\partial X^{A}} W^{A}-\frac{\partial \mathcal{L}}{\partial F^{a}{ }_{A}} \frac{\partial W^{B}}{\partial X^{A}} F_{B}^{a}=0 .
$$

If $\mathbf{W}$ is a constant, then

$$
\frac{\partial \mathcal{L}}{\partial X^{A}}=0
$$

i.e., the Lagrangian density must be materially homogeneous. This is also what Nelson ${ }^{35,36}$ ob- $^{3}$ tains. After some manipulation and assuming that Euler-Lagrange equations are satisfied (7.27) can be rewritten as

$$
\frac{\partial}{\partial X^{A}}\left(\mathcal{L} W^{A}-\frac{\partial}{\partial F^{a}{ }_{A}} F^{a}{ }_{B} W^{B}\right)-\frac{\partial}{\partial t}\left(\frac{\partial \mathcal{L}}{\partial \dot{\varphi}^{a}} F^{a}{ }_{A} W^{A}\right)-\mathcal{L} \frac{\partial W^{A}}{\partial X^{A}}=0,
$$

where use was made of the fact that $\mathbf{W}$ is time independent. For a volume-preserving flow this gives us 


$$
\frac{\partial}{\partial X^{A}}\left(\mathcal{L} W^{A}-\frac{\partial}{\partial F^{a}{ }_{A}} F_{B}^{a} W^{B}\right)-\frac{\partial}{\partial t}\left(\frac{\partial \mathcal{L}}{\partial \dot{\varphi}^{a}} F^{a}{ }_{A} W^{A}\right)=0,
$$

which is what Marsden and Hughes ${ }^{28}$ obtain. Now let us consider the general case of Riemannian manifolds and assume that $\Lambda_{s}$ is a flow on the Riemannian manifold $(\mathfrak{B}, \mathbf{G})$ generated by a vector field $\mathbf{W}$, i.e.,

$$
\left.\frac{d}{\mathrm{~d} s}\right|_{s=0} \Lambda_{s}(X)=\mathbf{W}(X), \quad X \in \mathcal{B} .
$$

Theorem 7.2: If the Lagrangian density is materially covariant then the following hold: (i) material homogeneity of the Lagrangian density and (ii) material Doyle-Ericksen formula.

Proof: Invariance of $\mathcal{L}$ with respect to $\Lambda_{s}$ means that

$$
\begin{aligned}
\mathcal{L} & \left(\Lambda_{s}^{A}(X), \varphi^{a}, \dot{\varphi}^{a},\left[\left(\frac{\partial \Lambda_{s}}{\partial X}\right)^{-1}\right]_{A}^{B} F_{B}^{a}, g_{a b},\left[\left(\frac{\partial \Lambda_{s}}{\partial X}\right)^{-1}\right]_{A}^{C}\left[\left(\frac{\partial \Lambda_{s}}{\partial X}\right)^{-1}\right]_{B}^{D} G_{C D}\right) \\
& =\mathcal{L}\left(X^{A}, \varphi^{a}, \dot{\varphi}^{a}, F^{a}{ }_{A}, g_{a b}, G_{A B}\right) .
\end{aligned}
$$

Differentiating the above relation with respect to $s$ and evaluating the result at $s=0$, one obtains

$$
\frac{\partial \mathcal{L}}{\partial X^{A}} W^{A}-\frac{\partial \mathcal{L}}{\partial F^{a}{ }_{A}} \frac{\partial W^{B}}{\partial X^{A}} F_{B}^{a}-2 \frac{\partial \mathcal{L}}{\partial G_{D K}} G_{D C} \frac{\partial W^{C}}{\partial X^{K}}=0 .
$$

Note that

$$
-\frac{\partial \mathcal{L}}{\partial F^{a}{ }_{A}} \frac{\partial W^{B}}{\partial X^{A}} F_{B}^{a}=-\left(F_{B}^{a} \frac{\partial \mathcal{L}}{\partial F^{a}{ }_{A}} W^{B}\right)_{\mid A}+\left(F_{B}^{a} \frac{\partial \mathcal{L}}{\partial F^{a}{ }_{A}}\right)_{\mid A} W^{B}+F^{a}{ }_{C} \frac{\partial \mathcal{L}}{\partial F^{a}{ }_{A}} \Gamma_{A B}^{C} W^{B}
$$

and

$$
-2 \frac{\partial \mathcal{L}}{\partial G_{D K}} G_{D C} \frac{\partial W^{C}}{\partial X^{K}}=-\left(2 \frac{\partial \mathcal{L}}{\partial G_{D K}} G_{D C} W^{C}\right)_{\mid K}+\left(2 \frac{\partial \mathcal{L}}{\partial G_{D K}} G_{D C}\right)_{\mid K} W^{C}+2 \frac{\partial \mathcal{L}}{\partial G_{D K}} G_{B D} \Gamma_{C K}^{B} W^{C} .
$$

Also

$$
\left(F^{a}{ }_{B} \frac{\partial \mathcal{L}}{\partial F^{a}{ }_{A}}\right)_{\mid A} W^{B}=\left(\frac{\partial F_{B}^{a}}{\partial X^{A}}-F^{a}{ }_{C} \Gamma_{B A}^{C}\right) \frac{\partial \mathcal{L}}{\partial F^{a}{ }_{A}} W^{B}+F^{a}{ }_{A}\left[\left(\frac{\partial \mathcal{L}}{\partial F^{a}{ }_{B}}\right)_{\mid B}+\frac{\partial \mathcal{L}}{\partial F^{b}{ }_{B}} F_{B}^{c} \gamma_{a c}^{b}\right] W^{A}
$$

Assuming that Euler-Lagrange equations are satisfied and using the above identities after a lengthy series of simplifications, one obtains

$$
\left(\mathcal{L} W^{A}-\frac{\partial \mathcal{L}}{\partial F^{a}{ }_{B}} W^{B}\right)_{\mid A}-\frac{\partial}{\partial t}\left(F^{a}{ }_{A} \frac{\partial \mathcal{L}}{\partial \dot{\varphi}^{a}} W^{A}\right)-\mathcal{L} W^{A}{ }_{\mid A}-\left(2 \frac{\partial \mathcal{L}}{\partial G_{D K}} G_{D C} W^{C}\right)_{\mid K}+\left(2 \frac{\partial \mathcal{L}}{\partial G_{D K}} G_{D C}\right)_{\mid K} W^{C}=0 .
$$

Note that

$$
-\left(2 \frac{\partial \mathcal{L}}{\partial G_{D K}} G_{D C} W^{C}\right)_{\mid K}+\left(2 \frac{\partial \mathcal{L}}{\partial G_{D K}} G_{D C}\right)_{\mid K} W^{C}=-2 \frac{\partial \mathcal{L}}{\partial G_{D K}} G_{D C} W_{\mid K}^{C}
$$

Therefore in this case Noether's theorem states that 


$$
\left(\mathcal{L} W^{A}-\frac{\partial \mathcal{L}}{\partial F_{B}^{a}} W^{B}\right)_{\mid A}-\frac{\partial}{\partial t}\left(F_{A}^{a} \frac{\partial \mathcal{L}}{\partial \dot{\varphi}^{a}} W^{A}\right)-\mathcal{L} W^{A}{ }_{\mid A}-2 \frac{\partial \mathcal{L}}{\partial G_{D K}} G_{D C} W_{\mid K}^{C}=0 .
$$

Note that

$$
\left(F_{B}^{a}{ }_{B} \frac{\partial \mathcal{L}}{\partial F_{A}^{a}} W^{B}\right)_{\mid A}=\frac{\partial}{\partial X^{A}}\left(F^{a}{ }_{B} \frac{\partial \mathcal{L}}{\partial F^{a}{ }_{A}}\right) W^{B}+F^{a}{ }_{B} \frac{\partial \mathcal{L}}{\partial F^{a}{ }_{A}} W^{B}+F^{a}{ }_{B} \frac{\partial \mathcal{L}}{\partial F^{a}{ }_{A}}\left(W^{B} \Gamma_{A C}^{C}-W^{C} \Gamma_{C A}^{B}\right) .
$$

Using the above relation and some lengthy simplifications, one can rewrite (7.39) as

$$
\left[\frac{\partial \mathcal{L}}{\partial X^{A}}+\left(\frac{\partial \mathcal{L}}{\partial F^{a}{ }_{C}} F_{B}^{a}+2 \frac{\partial \mathcal{L}}{\partial G_{C D}} G_{B C}\right) \Gamma_{A C}^{B}\right] W^{A}-\left(\frac{\partial \mathcal{L}}{\partial F^{a}{ }_{A}} F_{B}^{a}+2 \frac{\partial \mathcal{L}}{\partial G_{A C}} G_{B C}\right) W^{B}=0 .
$$

If $\mathcal{L}$ is materially covariant, i.e., if $\mathbf{W}$ is arbitrary, then

$$
\begin{gathered}
\frac{\partial \mathcal{L}}{\partial X^{A}}+\left(\frac{\partial \mathcal{L}}{\partial F^{a}{ }_{C}} F_{B}^{a}+2 \frac{\partial \mathcal{L}}{\partial G_{C D}} G_{B C}\right) \Gamma_{A C}^{B}=0, \\
\frac{\partial \mathcal{L}}{\partial F^{a}{ }_{A}{ }^{a}{ }_{B}+2 \frac{\partial \mathcal{L}}{\partial G_{A C}} G_{B C}=0 .}
\end{gathered}
$$

Or equivalently

$$
\begin{aligned}
& \frac{\partial \mathcal{L}}{\partial X^{A}}=0, \\
& \frac{\partial W}{\partial F^{a}{ }_{A}}{ }_{B}^{a}+2 \frac{\partial W}{\partial G_{A C}} G_{B C}=0,
\end{aligned}
$$

where $W$ is the material potential energy density. Note that (7.45) is nothing but the material Doyle-Ericksen formula (6.66).

Remarks: There are some differences between covariant energy balance (CEB) and Lagrangian density covariance (LDC):

(i) CEB is global while LDC is local.

(ii) In CEB the arbitrary vector fields $\mathbf{w}$ and $\mathbf{W}$ are time-dependent (being velocities), in general, while in LDC they are time independent.

(iii) In writing balance of energy in CEB for a material diffeomorphism spatial quantities contribute to energy balance. But in LDC a material flow does not affect the spatial quantities.

\section{CONCLUSIONS AND FUTURE DIRECTIONS}

The results of this paper can be summarized as follows.

(i) We studied continuum mechanics of bodies with global referential evolutions by enlarging the configuration manifold to two Riemannian manifolds with their own metrics. A deformation is then a pair of referential evolution, i.e., a motion in the referential manifold, and a standard motion. We showed that in the absence of discontinuities, configurational and standard equations of motion are equivalent even if the metrics are allowed to vary. 
(ii) The classical theorem of Green, Naghdi, and Rivilin ${ }^{19}$ was revisited and a material version of it was investigated. We showed that under a referential isometry balance of energy cannot be invariant, in general, and obtained its transformation.

(iii) The idea of covariance in elasticity was reviewed. We revisited a theorem by Marsden and Hughes ${ }^{28}$ and some of the details of its proof were clarified and a proof was given for its converse. It was also shown that spatial covariance of material energy balance leads to identical results.

(iv) We posed the question that whether energy balance can be materially covariant. It was shown that, in general, energy balance cannot be invariant under referential diffeomorphisms. We obtained the transformation of energy balance under arbitrary material diffeomorphisms. We found conditions under which energy balance is materially covariant. It was shown that in the absence of body forces the nontrivial condition for material covariance of balance of energy is equivalent to configurational stress tensor (Eshelby's stress tensor) being hydrostatic. It was shown that for ideal fluids energy balance is materially covariant.

(v) An explicit relation between covariance and Noether's theorem was found. We showed that spatial covariance of a Lagrangian density implies spatial homogeneity of the Lagrangian density and the Doyle-Ericksen formula. Similarly, material covariance of a Lagrangian density implies its material homogeneity and the material Doyle-Ericksen formula.

In summary, spatial covariance is reasonable and holds for most materials. The transformation properties of energy balance under material reframings was obtained. However, material covariance of energy balance only holds for special materials, such as ideal fluids.

The main application of the ideas presented in this paper will be in gaining a better understanding of the continuum theory of defects. In particular, if one repeats some of the developments presented in this paper in a space-time setting, one should, in principle, be able to obtain dynamic equations for evolution of defects. Another important relevant problem would be the study of covariance and its meaning in discrete systems. This may lead to a better understanding of "stress" in discrete systems.

\section{ACKNOWLEDGMENTS}

The authors have benefited from discussions with M. Arroyo, K. Bhattacharya, M. Desburn, E. Kanso, J. K. Knowles, J. Lu, P. M. Mariano, P. Papadopoulos, Y. Tong, and M. Zielonka. The research of one of the authors (J.E.M) was partially supported by the California Institute of Technology and NSF-ITR Grant No. ACI-0204932.

${ }^{1}$ Abeyaratne, R. and Knowles, K., "On the driving traction acting on a surface of strain discontinuity in a continuum," J. Mech. Phys. Solids 38, 345-360 (1990).

${ }^{2}$ Abeyaratne, R. and Knowles, K., "Kinetic relations and the propagation of phase boundaries in solids," Arch. Ration. Mech. Anal. 114, 119-154 (1990).

${ }^{3}$ Ariza, M. P. and Ortiz, M., "Discrete crystal elasticity and discrete dislocations in crystals," Arch. Ration. Mech. Anal. 178, 149-226 (2005).

${ }^{4}$ Batra, R. C., "The force on a lattice defect in an elastic body," J. Elast. 17, 3-8 (1987).

${ }^{5}$ Beig, R. and Schmidt, B. G., "Relativistic elasticity," Class. Quantum Grav. 20, 889-904 (2003).

${ }^{6}$ Conti, S. and Ortiz, M., "Dislocation microstructures and the effective behavior of single crystals, Arch. Ration. Mech. Anal. 176, 103-147 (2005).

${ }^{7}$ Doyle, T. C. and Ericksen, J. L., "Nonlinear elasticity," Adv. Appl. Mech. 4, 53-115 (1956).

${ }^{8}$ Epstein, M., "From saturated elasticity to finite evolution, plasticity and growth," Math. Mech. Solids 7, 255-283 (2002).

${ }^{9}$ Epstein, M. and Maugin, G. A., "The energy-momentum tensor and material uniformity in finite elasticity," Acta Mech. 83, 127-133 (1990)

${ }^{10}$ Ericksen, J. L., "Special topics in elastostatics," “Adv. Appl. Mech. 17, 189-224 (1976).

${ }^{11}$ Ericksen, J. L., "Remarks concerning forces on line defects," ZAMP 46, 247-271 (1995).

${ }^{12}$ Ericksen, J. L., "On nonlinear elasticity theory for crystal defects," Int. J. Plast. 14, 9-24 (1998).

${ }^{13}$ Eshelby, J. D., “The force on an elastic singularity," Philos. Trans. R. Soc. London, Ser. A 244, 87-112 (1951).

${ }^{14}$ Eshelby, J. D., "Energy relations and the energy-momentum tensor in continuum mechanics," Inelastic Behavior of Solids, edited by M. F. Kannien et al. (McGraw-Hill, New York, 1970). 
${ }^{15}$ Eshelby, J. D., "The elastic energy-momentum tensor,” J. Elast. 5, 321-335 (1975).

${ }^{16}$ Eshelby, J. D., "The force on a disclination in a liquid crystal," Philos. Mag. A 42, 359-367 (1980).

${ }^{17}$ Gotay, M. J., Isenberg, J., Marsden, J. E., and Montgomery, R., "Momentum maps and classical fields, Part I: Covariant field theory" (unpublished); see http://www.front.math.ucdavis.edu/physics/9801019.

${ }^{18}$ Gotay, M. J. and Marsden, J. E., "Stress-energy-momentum tensors and the Belinfante-Rosenfeld formula," Contemp. Math. 132, 367-392 (1991).

${ }^{19}$ Green, A. E. and Rivilin, R. S., “On Cauchy's equations of motion,” Z. Angew. Math. Phys. 15, 290-293 (1964).

${ }^{20}$ Gurtin, M. E., "On the nature of configurational forces," Arch. Ration. Mech. Anal. 131, 67-100 (1995).

${ }^{21}$ Gurtin, M. E., Configurational Forces as a Basic Concept of Continuum Physics (Springer-Verlag, New York, 2000).

${ }^{22}$ Knowles, J. K., "On the dissipation associated with equilibrium shocks in finite elasticity," J. Elast. 9, 131-158 (1979).

${ }^{23}$ Kröner, E., "Configurational and material forces in the theory of defects in ordered structures," Mater. Sci. Forum 123-125, 447-454 (1993).

${ }^{24}$ Lee, E. H., "Elastic-plastic deformation at finite strains," J. Appl. Mech. 36, 1-6 (1969).

${ }^{25}$ Lew, A., Marsden, J. E., Ortiz, M., and West, M., "Asynchronous variational integrators," Arch. Ration. Mech. Anal. 167, 85-146 (2003).

${ }^{26} \mathrm{Lu}$, J. and Papadopoulos, P., "A covariant constitutive descroption of anisotropic non-linear elasticity," ZAMP 51, 204-217 (2000).

${ }^{27} \mathrm{Lu}$, J. and Papadopoulos, P., "Referential doyle-ericksen formulae for the eshelby tensor in nonlinear elasticity," ZAMP 54, 964-976 (2003).

${ }^{28}$ Marsden, J. E. and Hughes, J. R., Mathematical Foundations of Elasticity (Prentice Hall, New York, 1983) (reprinted by Dover, New York, 1994).

${ }^{29}$ Marsden, J. E. and Ratiu, T., Introduction to Mechanics and Symmetry (Springer, New York, 2003).

${ }^{30}$ Marsden, J. E., Ratiu, T., and Abraham, R., Manifolds, Tensor Analysis and Applications (Oxford, New York, 2001).

${ }^{31}$ Maugin, G. A., Material Inhomogeneities in Elasticity (Chapman and Hall, New York, 1993).

${ }^{32}$ Maugin, G. A., "Material forces: concepts and applications," Appl. Mech. Rev. 48, 213-245 (1996).

${ }^{33}$ Maugin, G. A. and Trimarco, C., "Pseudomomentum and material forces in nonlinear elasticity: variational formulations and application to brittle fracture," Acta Mech. 94, 1-28 (1992).

${ }^{34}$ Nabarro, F. R. N., "Material forces and configurational forces in the interaction of elastic singularities," Proc. R. Soc. London, Ser. A 398, 209-222 (1975).

${ }^{35}$ Nelson, D. F., Electric, Optic, and Acoustic Interactions in Dielectrics (Wiley, New York, 1979).

${ }^{36}$ Nelson, D. F., "Momentum, pseudomomentum, and wave momentum: Toward resolving the Minkowski-Abraham controversy," Phys. Rev. A 44, 3985-3996 (1991).

${ }^{37}$ Rice, J. R., "A path-independent integral and the approximate analysis of strain concentration by notches and cracks," J. Appl. Mech. 35, 379-386 (1968).

${ }^{38}$ Shield, R. T., "Inverse deformation in finite elasticity," Z. Angew. Math. Phys. 18, 490-500 (1967).

${ }^{39}$ Šilhavý, M., "Mass, internal energy, and Cauchy's equations in frame-indifferent thermodynamics," Arch. Ration. Mech. Anal. 107, 1-22 (1989).

${ }^{40}$ Simo, J. C. and Marsden, J. E., "On the rotated stress tensor and the material version of the Doyle-Ericksen formula," Arch. Ration. Mech. Anal. 86, 213-231 (1984).

${ }^{41}$ Simo, J. C., Marsden, J. E., and Krishnaprasad, P. S., "The Hamiltonian structure of nonlinear elasticity: the material and convective representations of solids, rods, and plates," Arch. Ration. Mech. Anal. 104, 125-183 (1988).

${ }^{42}$ Steinman, P., "On spatial and material settings of hyperelastostatic crystal defects," J. Mech. Phys. Solids 50, 1743-1766 (2002).

${ }^{43}$ Steinman, P., "On spatial and material settings of thermo-hyperelastodynamics," J. Elast. 66, 109-157 (2002). 